\title{
Strong Decays of Strange Quarkonia
}

\author{
T. Barnes ${ }^{1,2 *}$, N. Black ${ }^{2 \dagger}$ and P.R. Page ${ }^{3 \ddagger}$ \\ ${ }^{1}$ Physics Division, Oak Ridge National Laboratory, \\ Oak Ridge, TN 37831-6373 \\ ${ }^{2}$ Department of Physics and Astronomy, University of Tennessee, \\ Knoxville, TN 37996-1501 \\ ${ }^{3}$ Theoretical Division, Los Alamos National Laboratory, \\ Los Alamos, NM 87545
}

Aug. 2002

\begin{abstract}
In this paper we evaluate strong decay amplitudes and partial widths of strange mesons (strangeonia and kaonia) in the ${ }^{3} \mathrm{P}_{0}$ decay model. We give numerical results for all energetically allowed open-flavor two-body decay modes of all $n \bar{s}$ and $s \bar{s}$ strange mesons in the $1 \mathrm{~S}, 2 \mathrm{~S}, 3 \mathrm{~S}$, $1 \mathrm{P}, 2 \mathrm{P}, 1 \mathrm{D}$ and $1 \mathrm{~F}$ multiplets, comprising strong decays of a total of 43 resonances into 525 two-body modes, with 891 numerically evaluated amplitudes. This set of resonances includes all strange $q \bar{q}$ states with allowed strong decays expected in the quark model up to $c a .2 .2 \mathrm{GeV}$. We use standard nonrelativistic SHO quark model wavefunctions to evaluate these amplitudes, and quote numerical results for all amplitudes present in each decay mode. We also discuss the status of the associated experimental candidates, and note which states and decay modes would be especially interesting for future experimental study at hadronic, $e^{+} e^{-}$and photoproduction facilities. These results should also be useful in distinguishing conventional quark model mesons from exotica such as glueballs and hybrids through their strong decays.
\end{abstract}

*email: barnes@bethe.phy.ornl.gov, tbarnes@utk.edu

†email: nblack@utk.edu

${ }^{\ddagger}$ email: prp@lanl.gov 


\section{Introduction}

Strange quarkonia are light $(u, d, s)$ mesons with at least one strange quark or antiquark in their dominant $q \bar{q}$ valence component. These are known as kaonia if the dominant valence basis state is $n \bar{s}$ (where $n \equiv u, d$ ), antikaonia if $s \bar{n}$, and strangeonia if $s \bar{s}$.

A principal goal of light meson spectroscopy is the identification of exotica, which are resonances that are not dominantly $q \bar{q}$ states. These include glueballs, hybrids, and multiquark systems. In the case of explicitly exotic quantum numbers, such as the $\mathrm{J}^{\mathrm{PC}}=1^{-+}$exotic $\pi_{1}(1600)$ resonance, exotica can be identified without a comparative study of the $q \bar{q}$ spectrum. Models of glueballs and hybrids predict however that the majority of light exotica will have nonexotic quantum numbers, and therefore must be identified against a background of conventional q $\bar{q}$ quark model mesons. In some cases, such as the scalar glueball, there is evidence of strong mixing between the gluonic basis state and $q \bar{q}$ quarkonium states. In these sectors it may be difficult to distinguish quarkonia from exotica, although the overpopulation of experimental resonances relative to the naive $q \bar{q}$ quark model will indicate the presence of the additional basis states.

Searches for the expected rich spectrum of exotica with nonexotic quantum numbers will require a well-established experimental meson spectrum over the relevant mass range of ca. 1.3-2.5 GeV, both to eliminate conventional quarkonium states and to study the possibility of a complicated pattern of mixing between exotica and conventional mesons.

The spectrum of meson resonances to $2 \mathrm{GeV}$ is only moderately well determined at present, and little is known regarding states above $2.2 \mathrm{GeV}$ in any light quark sector. The $n \bar{n}$ multiplets expected to $\approx 2.0 \mathrm{GeV}$ are $1 \mathrm{~S}, 2 \mathrm{~S}, 3 \mathrm{~S}, 1 \mathrm{P}, 2 \mathrm{P}, 1 \mathrm{D}$ and $1 \mathrm{~F}$, and of these 44 resonances, $c a$. 30 are now known. Of the 22 corresponding kaonia expected to $2.1 \mathrm{GeV}$, ca. 13 are known. Strangeonia in contrast are a terra incognita. Counting the maximally mixed $\eta-\eta^{\prime}$ as one $s \bar{s}$ state, only 7 probable $s \bar{s}$ resonances of the 22 expected to $2.2 \mathrm{GeV}$ are widely accepted, these being the $\eta-\eta^{\prime}, \phi(1019), h_{1}(1386), f_{1}(1426)$, $f_{2}^{\prime}(1525), \phi(1680)$ and $\phi_{3}(1854)$. As we shall see, there are controversies regarding the nature of two of these states as well.

In this paper we give detailed theoretical predictions for the strong decay amplitudes of two-body decay modes of all the strange mesons expected in the quark model to $c a$. $2.2 \mathrm{GeV}$. These decay amplitudes and partial and total widths are derived in the ${ }^{3} \mathrm{P}_{0}$ model, which (in several variants) is the standard model for strong decays. Since most experiments will rely on strong decay modes and amplitudes to identify and classify meson resonances, we have derived decay amplitudes to all openflavor two body modes that are nominally accessible. These results should be of use in establishing strange $q \bar{q}$ mesons, as well as in the identification of non- $q \bar{q}$ exotica.

Our results are presented in detailed tables of decay amplitudes, with entries for each resonance, decay mode and amplitude. We also include a short discussion of each quark model state and associated experimental candidates in the text, and where possible we compare our theoretical decay amplitudes to the data. We also note especially interesting theoretical and experimental results.

In most cases we assume pure $q \bar{q}$ mesons with definite $\mathrm{J}, \mathrm{L}$ and $\mathrm{S}$ as both initial and final states. In some cases, such as kaonia with $\mathrm{J}=\mathrm{L}$, spectroscopic mixing is allowed and is known experimentally to be important, so we give results as functions of mixing angles. Finally, in certain channels such as $0^{-+}$and $0^{++}\left(\eta-\eta^{\prime}\right.$ and $f_{0}$ states $)$ mixing between basis states of different flavor appears to be a large effect, and our results in these channels should be interpreted as decay amplitudes for initial ideal basis states, intended as a reference for contrast with experimental decays of the more complicated mixed states.

The organization of the paper is as follows: After this introduction we summarize the ${ }^{3} \mathrm{P}_{0}$ decay model used here; some additional technical details of the calculations are discussed in an appendix. 
After the decay model we discuss decays of strangeonia, and consider the status of states and give decay predictions for all states in the $1 \mathrm{~S}, 2 \mathrm{~S}, 3 \mathrm{~S}, 1 \mathrm{P}, 2 \mathrm{P}, 1 \mathrm{D}$ and $1 \mathrm{~F}$ multiplets, in that order. The following section carries out this exercise for kaonia. Our numerical results for these decay amplitudes and widths are presented in extensive decay tables following the text. Finally we give our summary and conclusions, and suggest topics of interest for future studies of strong decays.

\section{The Decay Model}

We employ the ${ }^{3} \mathrm{P}_{0}$ decay model with $\mathrm{SHO} q \bar{q}$ wavefunctions to evaluate two-body open-flavor strong decay amplitudes and widths. This model of strong decays was introduced over thirty years ago by Micu [1], and was applied extensively to meson decays in the 1970s by LeYaouanc et al. [2]. This decay model assumes that strong decays take place through the production of a $q \bar{q}$ pair with vacuum quantum numbers $\left(0^{++}\right.$, which corresponds to the ${ }^{3} \mathrm{P}_{0}$ state of a $q \bar{q}$ pair). After pair creation the $q^{2} \bar{q}^{2}$ system separates into two mesons in all possible ways, which corresponds to the two decay diagrams shown in Fig.A1 of Appendix A. Hairpin diagrams are assumed absent, and in any case would not be allowed by momentum conservation in this version of the ${ }^{3} \mathrm{P}_{0}$ model.

Since this model predates QCD and has no clear relation to it, one might expect that a description of decays in terms of allowed QCD processes such as one gluon exchange (OGE) might be more realistic. There is strong experimental evidence that the $q \bar{q}$ pair created during the decay does have spin one $\left(S_{q \bar{q}}=1\right)$, as is assumed in both the ${ }^{3} \mathrm{P}_{0}$ and OGE decay models. The strong experimental upper limit on the decay $\pi_{2}(1670) \rightarrow b_{1} \pi$ (from the VES Collaboration [3, 团) of

$$
B_{\pi_{2}(1670) \rightarrow b_{1} \pi}<1.9 \cdot 10^{-3}, \quad 97.7 \% \text { c.l. }
$$

provides striking evidence in favor of $S_{q \bar{q}}=1$. In the $q \bar{q}$ quark model this is a $1^{1} \mathrm{D}_{2} \rightarrow 1^{1} \mathrm{P}_{1}+1^{1} \mathrm{~S}_{0}$ transition, and any $\left(q_{i} \bar{q}_{i}\right) \rightarrow\left(q_{i} \bar{q}_{f}\right)\left(q_{f} \bar{q}_{i}\right)$ transition from a spin-singlet to spin-singlets has a vanishing matrix element if the $q_{f} \bar{q}_{f}$ pair is created with spin one.

A recent detailed theoretical study of light meson decays from OGE pair production [5] found that OGE decay amplitudes were typically rather smaller than required by experiment (the single exception found was $\left.1^{3} \mathrm{P}_{0} \rightarrow{ }^{1} \mathrm{~S}_{0}+{ }^{1} \mathrm{~S}_{0}\right)$ and hence are presumably masked by a dominant, nonperturbative decay mechanism. In addition, in certain decay amplitude ratios such as the $\mathrm{D} / \mathrm{S}$ ratios in $b_{1} \rightarrow \omega \pi$ (recently remeasured by the E852 Collaboration [6]) and $a_{1} \rightarrow \rho \pi$ there is a clear preference for $q \bar{q}$ production from a ${ }^{3} \mathrm{P}_{0}$ rather than an OGE source [7].

It is widely assumed that the ${ }^{3} \mathrm{P}_{0}$ model is successful because it gives a reasonably accurate description of a nonperturbative $q \bar{q}$ pair production mechanism, such as breaking of the gluonic flux tube between quark and antiquark sources through production of a new $q \bar{q}$ pair along the path of the flux tube. Presumably, future studies of lattice QCD will lead to a more fundamental description of this strong decay process. Here we simply assume the ${ }^{3} \mathrm{P}_{0}$ model, because of its success as an approximate description of much of the experimental data on strong decays.

Although the ${ }^{3} \mathrm{P}_{0}$ model is difficult to justify theoretically, it apparently does give a good description of many of the observed decay amplitudes and partial widths of open-flavor meson strong decays. There have been many references published on the decays of light, strange mesons using variants of the ${ }^{3} \mathrm{P}_{0}$ model (see Table 目) with different choices for the meson wavefunctions, the treatment of phase space, and the details of the ${ }^{3} \mathrm{P}_{0} q \bar{q}$ source. The flux-tube decay model [8, 9] is one well-known generalization of the ${ }^{3} \mathrm{P}_{0}$ model, in which the source strength is assumed to be largest along a path connecting the initial quark and antiquark. 


\begin{tabular}{|c|c|c|c|c|c|}
\hline Reference & Initial mesons considered & Amps. & Waves & p.s. & Wfns. \\
\hline this worl & 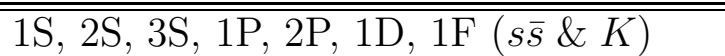 & 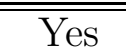 & 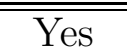 & 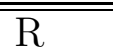 & SHO \\
\hline Bur00a [10] & $\begin{array}{l}f_{2}^{\prime}(1525), f_{2}(2010), f_{\mathrm{J}}(2220), f_{2}(2150), \\
f_{2}(2300), f_{2}(2340)\end{array}$ & No & No & $\mathrm{R}, \mathrm{M}$ & $\mathrm{SHO}$ \\
\hline Bur00b 11 & $h_{1}(1386), 2^{1} \mathrm{P}_{1} s \bar{s}$ & No & Yes & $\mathrm{R}, \mathrm{M}$ & $\mathrm{SHO}$ \\
\hline Bur98 12 & $1^{3} \mathrm{P}_{0} s \bar{s}$ & Yes & Yes & $\mathrm{R}, \mathrm{M}$ & $\mathrm{SHO}$ \\
\hline Str98 13 & $f_{0}(1370), f_{0}(1500), f_{0}(1710)$ & $\mathrm{No}$ & No & $\mathrm{R}$ & $\mathrm{SHO}$ \\
\hline Rob98 14 & $\begin{array}{l}\phi, f_{1}(1510), f_{2}^{\prime}(1525), 1^{3} \mathrm{P}_{0} s \bar{s}, \phi(1680), \\
(1 \mathrm{~S}, 2 \mathrm{~S} \& 1 \mathrm{P}) K, K_{2}(1580), K^{*}(1717) \\
K_{2}(1773), K_{3}^{*}(1776)\end{array}$ & No & Yes & $\mathrm{R}, \mathrm{M}$ & $\mathrm{O}$ \\
\hline Ams96 15 & $K_{0}^{*}(1412), 1^{3} \mathrm{P}_{0} s \bar{s}$ & No & Yes & $\mathrm{R}$ & $\mathrm{SHO}$ \\
\hline Blu96a 16 & $\begin{array}{l}\phi, f_{2}^{\prime}(1525), K^{*}, K_{0}^{*}(1412), K_{2}^{*}(1429) \\
K_{3}^{*}(1776), 1^{3} \mathrm{~F}_{2} \text { and } 1^{3} \mathrm{~F}_{4} s \bar{s}, K_{4}^{*}(2045)\end{array}$ & No & Yes & $\mathrm{R}, \mathrm{M}$ & $\mathrm{SHO}, \mathrm{O}$ \\
\hline Blu96b [17 & $K_{1}(1273)$ and $K_{1}(1402)$ & Yes & Yes & $\mathrm{M}$ & $\mathrm{SHO}, \mathrm{O}$ \\
\hline Kok87 9 & $1 \mathrm{~S}, 2 \mathrm{~S}, 1 \mathrm{P}, 2 \mathrm{P}, 1 \mathrm{D}, 1^{3} \mathrm{~F}_{4}(s \bar{s} \& K)$ & Yes & Yes & $\mathrm{M}$ & $\mathrm{SHO}, \mathrm{O}$ \\
\hline God85 18 & $1 \mathrm{~S}, 2 \mathrm{~S}, 1 \mathrm{P}, 1 \mathrm{D}, 1 \mathrm{~F}(s \bar{s} \& K)$ & Yes & Yes & $\mathrm{M}$ & $\mathrm{SHO}$ \\
\hline God84 & $1^{3} \mathrm{~F}_{2}$ and $1^{3} \mathrm{~F}_{4} s \bar{s}$ & No & No & $\mathrm{M}$ & $\mathrm{SHO}$ \\
\hline Bus83 20 & $\phi(1680)$ & No & No & $\mathrm{R}$ & $\mathrm{SHO}$ \\
\hline
\end{tabular}

Table 1: Some previous theoretical studies of strange meson decays in the literature. We indicate whether amplitudes are quoted, whether decay widths are displayed for the individual waves, and the phase space (relativistic (R) or mock meson (M)) and wavefunctions used (simple harmonic oscillator (SHO) or other $(\mathrm{O}))$.

We assume a fixed ${ }^{3} \mathrm{P}_{0}$ source strength (equivalent to the nonrelativistic limit of an $\mathcal{L}_{I}=g \bar{\psi}_{q} \psi_{q}$ pair production interaction Lagrangian [5]), simple harmonic oscillator (SHO) quark model meson wavefunctions, and physical (relativistic) phase space. The procedures we use to evaluate decay amplitudes and partial widths in this model are discussed in detail in [5] and in [21]; this paper is basically an application of the methods of the latter reference to the strange sector. The decay model parameters assumed here (in the notation of Ref.[21]) are $q \bar{q}$ pair production amplitude $\gamma=0.4$ and SHO wavefunction scale parameter $\beta=0.4 \mathrm{GeV}$. We assume physical, charge-averaged PDG values for the meson masses when there are clear and relatively uncontroversial candidates for states, and otherwise use an estimated mass, based where possible on known states in the same multiplet or in the nonstrange flavor sectors. Further details of the decay calculations are presented in Appendix A.

We use the ${ }^{3} \mathrm{P}_{0}$ decay model to evaluate all decay amplitudes and partial and total widths numerically for all the energetically allowed open-flavor two body modes of all expected 1S, 2S, 3S, 1P, 2P, $1 \mathrm{D}$ and $1 \mathrm{~F} s \bar{s}$ and $n \bar{s}$ states. This is the most complete survey of strange meson decays presented in the literature to date. For reference, in Table 1 we summarize previous strange meson strong decay calculations.

Since we use a narrow resonance approximation, one should interpret our predictions carefully for modes that are close to nominal thresholds. Some near-threshold modes that are energetically forbidden may actually have significant branching fractions when width effects are included, as is noted in our discussions in several important cases. One should also note that amplitudes with large orbital angular momenta between the final state mesons are often very sensitive to phase space, and hence to the assumed meson masses. 


\section{Strangeonia}

\subsection{General aspects}

The study of strangeonia should enter a new era with the advent of the new Hall D photoproduction facility GlueX at Jefferson Lab and the future upgraded $e^{+} e^{-}$facilities VEPP (Novosibirsk) and DAPHNE (Frascati). In interactions with hadrons a photon beam can be regarded as a superposition of vector mesons with an important $s \bar{s}$ component, so studies of strange final states at GlueX should lead to considerable improvement in our knowledge of the $s \bar{s}$ spectrum. The study of diffractive photoproduction reactions, $\gamma p \rightarrow X p$, should lead to the observation of many $\mathrm{C}=(-) s \bar{s}$ states. At $e^{+} e^{-}$facilities one of course makes only $1^{--}$states significantly, which will provide an extremely interesting case study of a pure $\mathrm{J}^{\mathrm{PC}}$ sector with broad overlapping resonances, presumably including vector hybrids as well as quarkonia. Central production has been shown at CERN and Fermilab to be very effective in the production of candidate $s \bar{s}$ states such as axial vectors, and it may be possible to use the STAR detector at RHIC similarly to study $s \bar{s}$ spectroscopy using pomeron and photon processes.

In previous experimental studies, strangeness-exchange reactions such as $K^{-} p \rightarrow X \Lambda$ were used as strangeonium production mechanisms. Unfortunately many of the more well-studied hadronic reactions, such as $\pi^{-} p$, have relatively weak $s \bar{s}$ production cross sections.

Surprisingly little is known about the strangeonium sector experimentally, due largely to the weakness of experimentally accessible $s \bar{s}$ production cross sections. Only three well-established resonances have been shown to be dominantly $s \bar{s}$, these being the $\phi, f_{2}^{\prime}(1525)$ and $\phi_{3}(1854)$. (Negative searches or confirmations of weak branching fractions to nonstrange final states are required to confirm $s \bar{s}$ dominance.) In this paper we hope to assist future searches for strangeonia by giving detailed predictions for the strong decay amplitudes of all $s \bar{s}$ mesons expected below ca. $2.2 \mathrm{GeV}$. For these calculations we employ the standard ${ }^{3} \mathrm{P}_{0}$ decay model, combined with $\mathrm{SHO}$ wavefunctions. This model has been tested extensively in decays of light nonstrange mesons, and is known to reproduce the qualitative features of most strong decays reasonably well, including relative amplitudes in several well-known test cases. Although the model is not derived from QCD and is therefore of unknown accuracy in its predictions in novel decay channels, it is the most accurate description of strong decays available, and its predictions should at least serve as a useful guide in the search for higher-mass states. Once the model proves to be accurate in a given channel, one can presumably trust the predictions in other flavor partners of that channel. Alternatively, a clear failure of the model may lead to important insights into the still poorly understood mechanism of strong decays.

Although we consider all allowed open-flavor two-body decay modes, some of these are especially characteristic of $s \bar{s}$ states. Due to the OZI rule, the observation of a state with a large branching fraction to $\eta \phi, \eta^{\prime} \phi$ or $\phi \phi$ and small branches to nonstrange final states can serve as a "smoking gun" for an initial $s \bar{s}$ state. (This rule may need modification if gluonia are nearby in mass, as in the scalar sector.) The mode $\eta \phi$ is particularly attractive for identifying $\mathrm{C}=(-) s \bar{s}$ candidates, and we strongly advocate the study of this final state in future experiments. We emphasize that decays to open-strangeness final states such as $K K, K K^{*}$ and $K^{*} K^{*}$ in isolation do not uniquely identify strangeonia, since light-quark isosinglet mesons $((u \bar{u}+d \bar{d}) / \sqrt{2})$ also decay to these open-strangeness final states.

One might naively expect the higher-mass $s \bar{s}$ spectrum to simply replicate the $n \bar{n}$ spectrum, ca. 200-250 MeV higher in mass. There is already considerable evidence that this is not the case. First, the near complete mixing of $n \bar{n}$ and $s \bar{s}$ states in the $\eta$ and $\eta^{\prime}$ is very well established. Second, there is circumstantial evidence that states in the scalar sector experience important $n \bar{n} \leftrightarrow G \leftrightarrow s \bar{s}$ 
mixing, specifically in the unusual decay branching fractions of the three states $f_{0}(1370), f_{0}(1500)$ and $f_{0}(1710)$. Similarly, the two known isosinglet $2^{-+}$states $\eta_{2}(1645)$ and $\eta_{2}(1870)$ are both observed in central production by WA102 [22], with comparable cross sections into the nonstrange final state $\pi a_{2}$. This suggests strong $n \bar{n} \leftrightarrow s \bar{s}$ mixing in the $2^{-+}$sector as well. Thus we may find that the spectrum of states with hidden strangeness is rather more complicated than a simple unmixed $s \bar{s}$ picture would suggest, due to channel-dependent annihilation couplings of $n \bar{n}$ and $s \bar{s}$ basis states.

\section{$3.2 \quad 1 S$ States}

There is a well-known problem with the decays of the lightest mesons that have allowed $1 \mathrm{~S} \rightarrow 1 \mathrm{~S}+1 \mathrm{~S}$ strong transitions, such as $\rho \rightarrow \pi \pi, K^{*} \rightarrow \pi K$ (and here, $\phi \rightarrow K K$ ); if we use parameter values fitted to a representative set of higher-mass decays [5, 21], these $1^{3} \mathrm{~S}_{1} \rightarrow 1^{1} \mathrm{~S}_{0}+1^{1} \mathrm{~S}_{0}$ partial widths are clearly underestimated. For $\phi \rightarrow K K$ the predicted and observed widths (Table S1) are $\Gamma_{t h y}=2.47 \mathrm{MeV}$ and $\Gamma_{\text {expt }}=4.26 \pm 0.05 \mathrm{MeV}$ 近, so $\Gamma_{\text {thy }} / \Gamma_{\text {expt }}=0.58$. Similarly, for the $\mathrm{SU}(3)$ partner decays $K^{*} \rightarrow \pi K$ and $\rho \rightarrow \pi \pi$ we find $\Gamma_{t h y} / \Gamma_{\text {expt }}=0.42$ and 0.32 respectively. (These results follow from our standard parameter set $\gamma=0.4$ and $\beta=0.4 \mathrm{GeV}$.) The reason for this discrepancy relative to the decays of higher-mass states is not known; one possibility involving reverse time-ordered "Z-graph" diagrams has been discussed by Page, Swanson and Szczepaniak [23].

\section{$3.3 \quad 2 \mathrm{~S}$ States}

\subsection{1 $\phi(1680)$}

The $\phi(1680)$ is a natural candidate for the $s \bar{s}$ radial excitation of the $\phi(1019)$, given its mass of ca. $250 \mathrm{MeV}$ above the $2^{3} \mathrm{~S}_{1} n \bar{n}$ candidates $\rho(1465)$ and $\omega(1419)$ and the absence of an $\omega \pi \pi$ mode [4]. The observation of the $\phi(1680)$ in $K K$ and $K K^{*}$ is sometimes cited as evidence that this state is $s \bar{s}$. Of course this evidence is ambiguous, since $n \bar{n}$ states also populate these modes; indeed, there is a danger of confusion of a $\phi(1680)$ with an $n \bar{n}$ state such as the $\omega(1649)$ if one considers only openstrangeness decay modes. True evidence for $s \bar{s}$ would be the observation of large branching fractions to hidden strangeness modes such as $\eta \phi$, or weak branching fractions to all accessible nonstrange modes.

Historically there has been considerable confusion about the $\phi(1680)$, due in part to this ambiguity regarding the $n \bar{n}$ versus $s \bar{s}$ origin of neutral $K K$ final states [24]. The first report of this state was by the DM1 collaboration at DCI, in $e^{+} e^{-} \rightarrow K_{L} K_{S}$ [25], in which a rapid fall of the cross section was interpreted as due to a new vector, the $\phi(1650)$. Similar behavior in $e^{+} e^{-} \rightarrow K^{+} K^{-}$ was also speculatively attributed to a possible new vector meson by DM1 [26], and an excess of events in $e^{+} e^{-} \rightarrow K^{+} K^{-}$above $1.15 \mathrm{GeV}$ invariant mass was noted by the VEPP-2M collaboration in Novosibirsk [27]. This $e^{+} e^{-} \rightarrow K^{+} K^{-}$reaction was subsequently studied with slightly better statistics by DM2 [28], who assumed a $\phi(1680)$ to fit the cross section.

Observation of a much larger signal in $e^{+} e^{-} \rightarrow K_{S} K^{ \pm} \pi^{\mp}$ [29] motivated fits with interference between a $\phi(1680)$ and a $\rho^{\prime}$ (which was needed to explain the dominance of neutral over charged $K K^{*}$ states in this channel); the fitted $\phi(1680)$ parameters were $\mathrm{M}=1677 \pm 12 \mathrm{MeV}$ and $\Gamma=$ $102 \pm 36 \mathrm{MeV}$. A subsequent global fit by DM1 to these $K K$ and $K K \pi$ channels together with data on $e^{+} e^{-} \rightarrow \omega \pi \pi, \rho \pi, \rho \eta$ and $\rho \pi \pi$ in a $\omega^{\prime}-\rho^{\prime}-\phi^{\prime}$ model with interference gave $\phi(1680)$ resonance parameters $\mathrm{M}=1680 \pm 10 \mathrm{MeV}$ and $\Gamma=185 \pm 22 \mathrm{MeV}$ [30]. DM2 next studied the reactions $e^{+} e^{-} \rightarrow K K \pi$ [31] and $e^{+} e^{-} \rightarrow \omega \pi^{+} \pi^{-}$[32] with improved statistics, and generally confirmed the DM1 results. Their fitted $\phi(1680)$ parameters were $\mathrm{M}=1657 \pm 27 \mathrm{MeV}$ and $\Gamma=146 \pm 55 \mathrm{MeV}$, 
and interference between this state and a $\rho^{\prime}$ nearby in mass was again used to explain the dominance of neutral over charged $K K^{*}$ modes. A corresponding $\omega^{\prime}$ near $1650 \mathrm{MeV}$ was clearly evident in the $e^{+} e^{-} \rightarrow \omega \pi^{+} \pi^{-}$cross section (see Fig.6 of Ref.[32]). The only reported relative strong branching fraction for the $\phi(1680) e^{+} e^{-}$state, from DM1 [30], is

$$
B_{\phi(1680) \rightarrow K K / K K^{*}}=0.036 \pm 0.004 / 0.49 \pm 0.05 .
$$

The PDG quote this as $B_{\phi(1680) \rightarrow K K / K K^{*}}=0.07 \pm 0.01$.

Photoproduction experiments have reached rather different conclusions regarding the " $\phi(1680)$ ". The CERN Omega Spectrometer [33] found a $K^{+} K^{-}$enhancement in $\gamma p \rightarrow K^{+} K^{-} p$ centered at $\mathrm{M}_{K^{+} K^{-}} \approx 1.75 \mathrm{GeV}$ (see their Fig.4). A single Breit-Wigner fit gave the parameters $\mathrm{M}=1748 \pm$ $11 \mathrm{MeV}$ and $\Gamma=80 \pm 33 \mathrm{MeV}$. They noted however that interference effects can modify fitted resonance parameters, and in a model including interference with light vector meson tails a lower mass was found, $\mathrm{M}=1690 \pm 10 \mathrm{MeV}$ and $\Gamma=100 \pm 40 \mathrm{MeV}$. A second Omega Spectrometer study of this process by WA57 [34] advocated a single Breit-Wigner fit without interference, which gave a mass and width of $\mathrm{M}=1760 \pm 20 \mathrm{MeV}$ and $\Gamma=80 \pm 40 \mathrm{MeV}$, consistent with the earlier photoproduction result. Fermilab photoproduction experiment E401 [35] studied photoproduction of $K^{+} K^{-}$pairs at somewhat higher photon energies, and confirmed the $\approx 1750 \mathrm{MeV}$ enhancement; a Breit-Wigner fit gave the parameters $\mathrm{M}=1726 \pm 22 \mathrm{MeV}$ and $\Gamma=121 \pm 47 \mathrm{MeV}$. Finally, the FOCUS collaboration at Fermilab very recently reported a high-statistics study of diffractive photoproduction of $K^{+} K^{-}$ [36], and see a clear enhancement with a fitted mass and width of $\mathrm{M}=1753.5 \pm 1.5 \pm 2.3 \mathrm{MeV}$ and $\Gamma=122.2 \pm 6.2 \pm 8.0 \mathrm{MeV}$, again consistent with previous photoproduction experiments but with much smaller errors. The $K K^{*}$ channel is also studied, and there is no evidence for the $1750 \mathrm{MeV}$ enhancement; in the neutral $K K^{*}$ channel a limit of

$$
\frac{\Gamma_{X(1750) \rightarrow K^{o} K^{* o} \rightarrow \pi^{+} K^{-} K_{S}+h . c .}}{\Gamma_{X(1750) \rightarrow K^{+} K^{-}}}<0.065 \quad 90 \% \text { c.l. }
$$

is reported. Note that this is in striking disagreement with the $K K^{*}$ dominance found for the $\phi(1680)$ state seen in $e^{+} e^{-}$.

In summary, $e^{+} e^{-}$and photoproduction experiments typically find " $\phi(1680)$ " enhancements at masses that differ by $\approx 50-100 \mathrm{MeV}$, with $e^{+} e^{-}$reporting $K K^{*}$ dominance and photoproduction reporting $K K$ dominance. This may constitute evidence for two distinct states, although interference with $n \bar{n}$ vectors may complicate a comparison of these two processes. This issue can be addressed by studying channels in which interference with $n \bar{n}$ vectors is expected to be unimportant, notably $\eta \phi$, and by comparing the relative branching fractions to charged versus neutral modes in decays to $K K$ and $K K^{*}$.

In our decay calculations (Table S2) we find that $K K^{*}$ is predicted to be the dominant $2^{3} \mathrm{~S}_{1} s \bar{s}$ decay mode, as is observed for the $e^{+} e^{-}$state $\phi(1680)$. We actually predict a $K K / K K^{*}$ branching fraction ratio of $B_{\phi(1680) \rightarrow K K / K K^{*}} \approx 0.35$, rather larger than the experimental ratio $0.07 \pm 0.01$. Our $2^{3} \mathrm{~S}_{1} s \bar{s}$ decay predictions are in clear disagreement with the $K K$ dominance reported by FOCUS for the $X(1750)$; evidently this state is not consistent with the ${ }^{3} \mathrm{P}_{0}$ model predictions for a $2^{3} \mathrm{~S}_{1} s \bar{s}$ radial excitation.

The $\eta \phi$ mode should be useful in establishing the true mass and width of the $2^{3} \mathrm{~S}_{1} s \bar{s}$ state, since interference with nonstrange vectors should be unimportant in this channel. Our prediction of a branching fraction ratio of $B_{\phi(1680) \rightarrow \eta \phi / K K^{*}} \approx 0.18$ should be reliable, since these decays are controlled by the same amplitude, have similar phase space, and differ mainly through a flavor 
factor. We strongly encourage the study of the $\eta \phi$ channel in searches for evidence of a $2^{3} \mathrm{~S}_{1} s \bar{s}$ state in the " $\phi(1680) "$ region.

\subsection{2 $\quad 2^{1} \mathrm{~S}_{0} s \bar{s}$ and the $\eta(1440)$ region}

The $2{ }^{1} \mathrm{~S}_{0} s \bar{s}$ state should theoretically have quite simple strong decay properties, assuming that $\eta$ $\eta^{\prime}$ type flavor mixing is unimportant in the radially-excited states. The only open-flavor mode is $K K^{*}$, which is a $\mathrm{P}$-wave decay (Table $\mathrm{S} 2$ ). Since the $2^{1} \mathrm{~S}_{0} s \bar{s}$ state is presumably rather close to $K K^{*}$ threshold, we find a total width that varies strongly with mass; between $\mathrm{M}=1415 \mathrm{MeV}$ and $1500 \mathrm{MeV}$ the predicted width increases from 11 to $100 \mathrm{MeV}$. Since the experimental $\eta(1440)$ is reported to have an important $K K^{*}$ mode and has a total width (PDG estimate) of $50-80 \mathrm{MeV}$, it appears plausible as a $2^{1} \mathrm{~S}_{0} s \bar{s}$ candidate.

Unfortunately the $\eta(1440)$ suffers from many complications in the determination of its resonance parameters. One problem is that the S-wave $f_{1}(1426)$ signal is typically present in the same reactions, and the $0^{-+}$and $1^{++}$contributions are difficult to separate. Another problem is the strong $K K$ final state interaction, which distorts the $\eta(1440) \rightarrow K K^{*} \rightarrow K K \pi$ invariant mass distribution and leads to a low-mass $K K$ peak, which may be misidentified as a decay to $\pi a_{0}(980)$ [37. If there actually is a strong $\pi a_{0}(980)$ mode, what this tells us about the $\eta(1440)$ is unclear because the $a_{0}(980)$ itself is not well understood. Finally, there are suggestions of several $0^{-+}$isosinglet states near this mass, because fits to the $\eta \pi \pi$ and $K K \pi$ final states give somewhat different masses for the parent resonances [4]. Of course this might also be due to final state interactions or interferences that vary between channels. The recent evidence from E852 (BNL) 38] for two resonances $\eta(1415)$ and $\eta(1485)$ in the same decay channel, $K K^{*}$, may be more significant. If this is correct, the existence of the three states $\eta(1295)$, $\eta(1415)$ and $\eta(1485)$ suggests the presence of additional degrees of freedom beyond the two $\mathrm{I}=02^{1} \mathrm{~S}_{0}$ $q \bar{q}$ quark model states expected in this mass range.

The $\eta(1440)$ confusion may be dispelled through the study of different production mechanisms and decay modes. Possibilities include $\gamma \gamma$ production (these rates can be calculated in the quark model, and checked against well-established $q \bar{q}$ states in this mass region) and flavor-tagging radiative decays such as $\eta(1440) \rightarrow \gamma \rho^{o}, \gamma \omega$ and $\gamma \phi$.

There is a recent report from L3 [39] of a signal consistent with the $\eta(1440)$ in $\gamma \gamma \rightarrow K_{S} K^{ \pm} \pi^{\mp}$, with a two-photon width of

$$
\Gamma_{\gamma \gamma}(\eta(1440)) \cdot B_{K K \pi}=212 \pm 50 \pm 23 \mathrm{eV}[39]
$$

which is comparable to the larger of the theoretical expectations for the two-photon width of a $2{ }^{1} \mathrm{~S}_{0}$ $s \bar{s}$ state. (Scaling the Ackleh-Barnes result $\Gamma_{\gamma \gamma}(\pi(1300))=0.43-0.49 \mathrm{KeV}$ 40] by $2 / 9$ for flavor and $(1.44 / 1.3)^{3}$ for phase space gives $\Gamma_{\gamma \gamma}\left(2^{1} \mathrm{~S}_{0} s \bar{s}\right) \approx 140 \mathrm{eV}$. Similarly scaling the Münz $\pi(1300)$ results, which use three different models 41 , gives $\Gamma_{\gamma \gamma}\left(2^{1} \mathrm{~S}_{0} s \bar{s}\right) \approx 30-100 \mathrm{eV}$.)

Although little is known experimentally about the radiative transitions of any higher-mass states, there is an early Mark III report of a large $\eta(1440) \rightarrow \gamma \rho^{\circ}$ partial width [42 that, if confirmed, would invalidate the assumption that this is a relatively pure $s \bar{s}$ state. Measurements of the radiative partial widths of the $\eta(1440)$ and other states through high-statistics studies of $J / \psi \rightarrow \gamma \gamma V\left(V=\rho^{o}, \omega\right.$ and $\phi)$ would be very important experimental contributions, which should be feasible at CLEO-c.

Until such data become available, we can summarize the status of the $\eta(1440)$ (assuming that this is indeed a single state) by noting that the reported total width and two-photon partial width appear consistent with expectations for a $2^{1} \mathrm{~S}_{0} s \bar{s}$ state decaying dominantly to $K K^{*}$, but final state interactions may invalidate this agreement. 


\subsection{S States}

\subsubsection{The unobserved $\phi(2050)$}

The $3{ }^{3} \mathrm{~S}_{1} s \bar{s}$ vector state, to which we assign an estimated mass of $2.05 \mathrm{GeV}$, is not known at present. This state should be important in future spectroscopic studies, because with $1^{--}$quantum numbers it can be made both in diffractive photoproduction and in $e^{+} e^{-}$annihilation. A hybrid with the same quantum numbers and a similar mass is predicted by the flux-tube model [43, 44, so overpopulation of this sector may be anticipated.

The ${ }^{3} \mathrm{P}_{0}$ model predicts that this will be a rather broad state, $\Gamma_{t o t} \approx 380 \mathrm{MeV}$ (Table S3). In fluxtube decay models the corresponding $s \bar{s}$-hybrid is predicted to be much narrower, $\Gamma_{\text {tot }} \approx 100-150 \mathrm{MeV}$ [23, 45]. The dominant decay modes of the $3^{3} \mathrm{~S}_{1}$ state are predicted to be $K^{*} K^{*}, K K^{*}(1414)$ and $K K_{1}(1273)$, in order of decreasing branching fraction. All these lead to important $K K \pi \pi$ final states. The large branching fraction for the $3 \mathrm{~S} \rightarrow 1 \mathrm{~S}+2 \mathrm{~S}$ transition to $K K^{*}(1414)$ may appear surprising, since this decay amplitude has three nodes. These however are at $x=\left|\vec{p}_{f}\right| / \beta \approx 2.4,4.5$ and 7.5 , rather far from the physical $x \approx 0.4$, so there is no dramatic nodal suppression. Assuming that the decay model is accurate, it will be very interesting to see whether the problematical $K^{*}(1414)$ is indeed produced copiously in $\phi(2050)$ decay, as expected if the $K^{*}(1414)$ is the $2^{3} \mathrm{~S}_{1}$ state. Finally, the $K K$ mode is near a node in the ${ }^{3} \mathrm{P}_{0}$ decay amplitude, and so is predicted to be very weak.

A study of the $s \bar{s}$-signature modes $\eta \phi$ and $\eta^{\prime} \phi$ may be an effective experimental strategy for identifying this state. A $3{ }^{3} \mathrm{~S}_{1} s \bar{s} \phi(2050)$ is predicted to have significant branching fractions to both of these final states, whereas the decay couplings of any $n \bar{n}$ state to anything $+\phi$ should be weak. Close and Page [45] anticipate that the $s \bar{s}$-hybrid vector should also have a large $\eta \phi$ branching fraction, although the $\eta^{\prime} \phi$ mode of the hybrid should be weak.

We note in passing that since the $K^{*}$ and $\bar{K}^{*}$ are antiparticles, neutral $\left(K^{*} \bar{K}^{*}\right)^{o}$ final states of definite isospin have diagonal C-parity,

$$
C\left|K^{*} \bar{K}^{*}\right\rangle_{L, S, I}=(-)^{L+S+I}\left|K^{*} \bar{K}^{*}\right\rangle_{L, S, I}
$$

C-parity conservation forbids many transitions to VV states that one might expect to appear in the decay amplitude tables based on angular momentum alone. The two C-forbidden amplitudes here are $\phi(2050) \rightarrow K^{*} K^{*}\left({ }^{5} \mathrm{P}_{1}\right)$ and $\phi(2050) \rightarrow K^{*} K^{*}\left({ }^{5} \mathrm{~F}_{1}\right)$.

\subsubsection{The unobserved $\eta_{s}(1950)$}

The ${ }^{3} \mathrm{P}_{0}$ decay model predicts a relatively narrow ${ }^{1} \mathrm{~S}_{0} s \bar{s}$ state, with $\Gamma_{\text {tot }} \approx 175 \mathrm{MeV}$, decaying dominantly to $K K^{*}$ and $K^{*} K^{*}$ (Table S3). Experimental confirmation of this state may be difficult despite the moderate width, due to small production cross sections and the absence of characteristic $s \bar{s}$-signature decay modes such as $\eta \phi$. Nondiffractive photoproduction of this $\mathrm{C}=(+)$ state is expected to be weak, since $\gamma \rightarrow V$ followed by nonstrange t-channel $\mathrm{C}=(-)$ meson exchange does not lead to $s \bar{s}$ states (assuming the OZI rule). As an $s \bar{s}$ state, the $\eta_{s}(1950)$ will also have a small $\gamma \gamma$ coupling.

Radiative transitions from the $J / \psi$ may be a more appropriate technique for identifying the $\eta_{s}(1950)$, since $J / \psi \rightarrow \gamma \eta$ and $\gamma \eta^{\prime}$ are both known to have relatively large branching fractions, and no important $s \bar{s}$ suppression is expected in this process. Hadronic production of this state may also be effective in reactions with significant $s \bar{s}$ production cross sections. 


\begin{tabular}{|c|c|c|c|c|}
\hline mode: $\Gamma_{i}(\mathrm{MeV})$ & $K K$ & $K K^{*}$ & $\eta \eta$ & $\pi \pi$ \\
\hline \hline$f_{2}^{\prime}(1525)($ expt $)$ & $65_{-4}^{+5}$ & - & $7.6 \pm 2.5$ & $0.60 \pm 0.12$ \\
\hline$f_{2}^{\prime}(1525)$ (thy) & 61 & 8.6 & 10.4 & 0 \\
\hline
\end{tabular}

Table 2: Experimental and theoretical partial widths of the $f_{2}^{\prime}(1525)$. Note the unreported $K K^{*}$ mode.

\section{$3.51 P$ States}

3.5.1 $f_{2}^{\prime}(1525)$

This state is almost universally accepted as the $s \bar{s}$ member of the $1^{3} \mathrm{P}_{2} q \bar{q}$ flavor nonet, together with the $a_{2}(1318), f_{2}(1275)$ and $K_{2}^{*}(1429)$. Although $n \bar{n} \leftrightarrow s \bar{s}$ mixing is allowed in principle, in practice the $f_{2}^{\prime}(1525)$ appears to be close to pure $s \bar{s}$; the mixing angle is strongly constrained by the experimental $f_{2}^{\prime}(1525) \gamma \gamma$ coupling, which limits the $n \bar{n}$ content to a few percent.

Our decay model predictions are in good agreement with the reported total width of $76 \pm 10 \mathrm{MeV}$ (we predict $80 \mathrm{MeV}$ ) and the known partial widths, shown in Tables 2 and S4. There is a difficulty with this comparison, however, which is that the PDG gives partial widths assuming that only the modes $K K, \eta \eta$ and $\pi \pi$ contribute significantly. We find that the neglected mode $K K^{*}$ should actually be about as large as $\eta \eta$. There is only a weak experimental constraint on this mode at present, $B_{K K^{*}}<0.35$ at $95 \%$ c.l. [1].

\subsection{2 $f_{1}(1426), f_{1}(1510)$}

The status of axial-vector states in this mass region has long been confused, largely due to the overlap of important $0^{-+}, 1^{++}$and $1^{+-}$amplitudes in $K K \pi$ hadroproduction near $K K^{*}$ threshold. Although some studies of phase motion of these amplitudes have been reported [38, 46], the statistics to date have not been sufficient to extract convincing individual resonance phase shifts in the pseudoscalar or axial-vector channels.

Three light, $\mathrm{C}=(+)$ axial-vector isosinglets have been claimed experimentally, the $f_{1}(1285)$, $f_{1}(1426)$ and $f_{1}(1510)$. There is also evidence for the $f_{1}(1285)$ and $f_{1}(1426)$ in $J / \psi$ radiative decays and $\gamma \gamma^{*}$, and some rather more controversial evidence in $J / \psi$ hadronic decays. The various reports of axial-vector signals were summarized recently by Close and Kirk [47], who expressed skepticism regarding the existence of an $f_{1}(1510)$, and speculated that there might be significant $n \bar{n} \leftrightarrow s \bar{s}$ flavor mixing between the $f_{1}(1285)$ and $f_{1}(1426)$.

The historically confused experimental status of light axial vectors has improved considerably with high-statistics central production experiments on $\eta \pi \pi, K K \pi$ and $4 \pi$ states by WA102 (CERN) [48, 49] and $K K \pi$ by E690 (Fermilab) [50]. Central production of $K K \pi$ and $\eta \pi \pi$ in this mass region has been found to favor axial-vector quantum numbers strongly, and very clear $f_{1}(1285)$ and $f_{1}(1426)$ states are observed. There is no evidence of an $f_{1}(1510)$ in central production.

In view of their masses, the obvious assumption is that the $f_{1}(1285)$ is the light, dominantly $n \bar{n}$ $1^{3} \mathrm{P}_{1}$ state, and the $f_{1}(1426)$ is its dominantly $s \bar{s} 1^{3} \mathrm{P}_{1}$ partner. Since there is a controversy over the identification of the $f_{1}(1426)$ or the $f_{1}(1510)$ as the $1^{3} \mathrm{P}_{1} s \bar{s}$, in Fig. 1 we show the ${ }^{3} \mathrm{P}_{0}$-model total width prediction for a range of $1^{3} \mathrm{P}_{1} s \bar{s}$ masses. (The only open-flavor two-body mode below $1.77 \mathrm{GeV}$ is $K K^{*}$.) The nominal threshold is $1390 \mathrm{MeV}$, however as the $f_{1} \rightarrow K K^{*}$ decay is dominantly Swave we find that the width increases rapidly with increasing mass. At $\mathrm{M}=1420 \mathrm{MeV}$ the predicted width is $254 \mathrm{MeV}$, and the resonance envelope would obviously be strongly distorted by the nearby threshold, which is at a $\Delta E<<\Gamma_{\text {tot }}$. Other calculations of the $f_{1}(1426) \rightarrow K K^{*}$ width, also using 


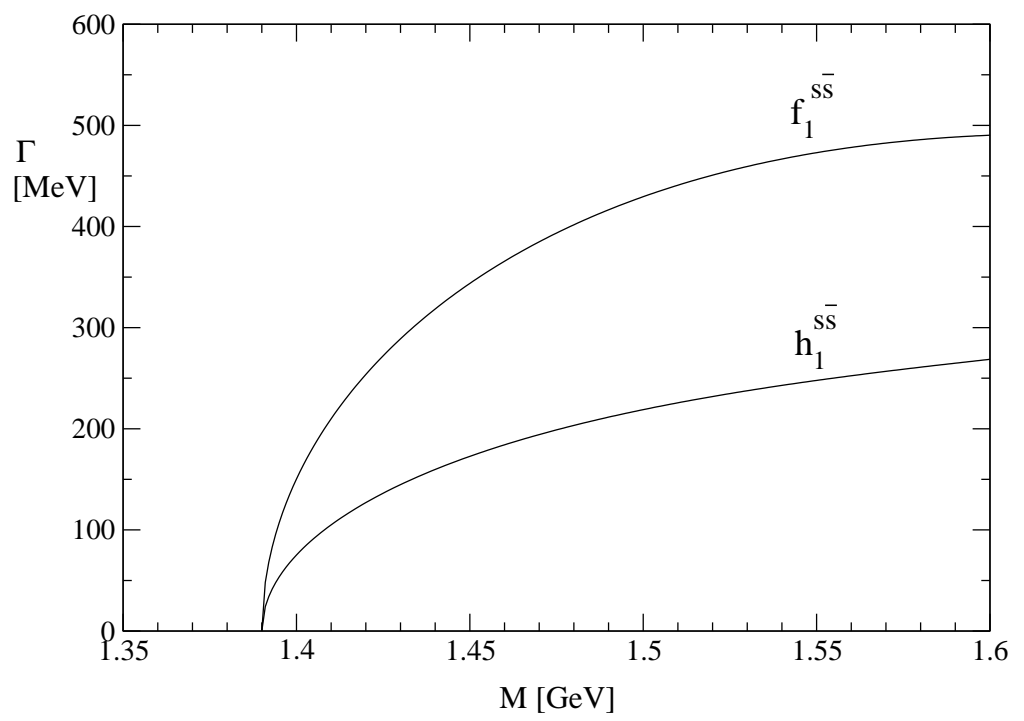

Figure 1. Theoretical $K K^{*}$ widths of $1^{3} \mathrm{P}_{1} f_{1}$ and $1^{1} \mathrm{P}_{1} h_{1} s \bar{s}$ states versus assumed mass.

the ${ }^{3} \mathrm{P}_{0}$ model but taking threshold modification of the Breit-Wigner resonance shape into account, quote effective widths of $\sim 70 \mathrm{MeV}$ [14] and $\sim 120 \mathrm{MeV}$ [9]. Thus the ${ }^{3} \mathrm{P}_{0}$ model is roughly consistent with the reported width of the $f_{1}(1426)$, given the uncertainties in modeling the effect of the nearby $K K^{*}$ threshold.

At the mass of $1530 \mathrm{MeV}$ reported by LASS [51], the theoretical width of a $1^{3} \mathrm{P}_{1} s \bar{s} f_{1}(1530)$ is a very large $459 \mathrm{MeV}$. Assuming the decay model is realistic for this channel, the relatively small reported width of $\Gamma=100 \pm 40 \mathrm{MeV}$ makes the $f_{1}(1530)$ appear implausible as a $1^{3} \mathrm{P}_{1} s \bar{s}$ state.

As the decay model predicts a quite strong coupling between the bare quark model $1^{3} \mathrm{P}_{1} s \bar{s}$ state and the $K K^{*}$ decay channel, and these are close to degenerate, it may be necessary to treat this as a coupled $s \bar{s}, n \bar{n}$ and $K K^{*}$ system. This concern applies to the $1^{1} \mathrm{P}_{1} s \bar{s}$ sector as well.

In future experimental work it will be important to test the expected resonant phase motion of the $f_{1}(1426)$ in channels in which this state and others are present with comparable amplitudes. This is especially important here because of the possibility of misinterpreting a nonresonant threshold enhancement as a resonant state.

Future accurate measurements of radiative transition rates of the $f_{1}(1426)$ and the other axial vectors will be of great importance in testing candidate $q \bar{q}$ assignments [52, 53, 54]. Transitions such as $f_{1}(1426) \rightarrow \gamma \rho$ and $\gamma \phi$ are flavor tagging, and will allow determinations of the amount of flavor mixing in the parent axial vectors. (This is especially interesting because Close and Kirk [47] cite evidence of important $n \bar{n} \leftrightarrow s \bar{s}$ mixing in the axial vector system.) The absolute radiative transition rates are among the simplest and presumably most reliable quark model predictions for $q \bar{q}$ mesons, so a set of accurate measurements of radiative partial widths to $\gamma \omega, \gamma \rho$ and $\gamma \phi$ could be definitive in establishing the nature of the axial vectors and other states in this mass region. A first measurement of the radiative transition $f_{1}(1426) \rightarrow \gamma \phi$ has been reported by WA102 [48], who quote a relative branching fraction of $B_{f_{1}(1426) \rightarrow \gamma \phi / K K \pi}=0.003 \pm 0.001 \pm 0.001$, corresponding to $\Gamma\left(f_{1}(1426) \rightarrow \gamma \phi\right) \sim 150 \mathrm{keV}$ (but clearly not yet well determined). Given the large errors, this may be consistent with the theoretical expectation of $\Gamma_{t h y}\left(f_{1}(1426) \rightarrow \gamma \phi\right) \approx 50 \mathrm{keV}$ for pure $s \bar{s}$ initial and final mesons [55]. Evidently experimental accuracies of $c a .10 \mathrm{keV}$ will be required for definitive radiative transition tests of $s \bar{s}$ quark model assignments. 


\begin{tabular}{|l|l||c|c|c|c||c|}
\hline State & Experiment & $\pi \pi$ & $K K$ & $\eta \eta$ & $\eta \eta^{\prime}$ & $4 \pi$ \\
\hline \hline$f_{0}(1500)$ & WA102 & 1 & $0.33 \pm 0.07$ & $0.18 \pm 0.003$ & $0.096 \pm 0.026$ & $1.36 \pm 0.15$ \\
& CBar & 1 & $0.184 \pm 0.025$ & $0.08 \pm 0.04$ & $0.065 \pm 0.008$ & $1.62 \pm 0.18$ \\
\hline$f_{0}(1710)$ & WA102 & 1 & $5.0 \pm 0.7$ & $2.4 \pm 0.6$ & $<0.18$ & $<5.4$ \\
\hline
\end{tabular}

Table 3: Experimental branching ratios for $f_{0}(1500)$ and $f_{0}(1710)$ from the WA102 [59] and Crystal Barrel [60] experiments, normalized to the $\pi \pi$ branching ratio.

\subsection{3 $f_{0}(1500)$ and $f_{0}(1710)$}

The scalar sector is of great interest, since LGT predicts that the lightest glueball is a scalar with a mass near $1.7 \mathrm{GeV}$ [56] (neglecting decays and mixing with quarkonia). We also expect $1^{3} \mathrm{P}_{0} n \bar{n}$ and $s \bar{s}$ quark model scalars at masses of $\sim 1.4 \mathrm{GeV}$ and $\sim 1.6 \mathrm{GeV}$ respectively, so the $\mathrm{I}=00^{++}$ sector may be expected to show evidence of overpopulation relative to the $q \bar{q}$ quark model in this mass region.

Ideally we might hope to distinguish a glueball from quarkonia through anomalous decay or production amplitudes. Assuming unmixed $f_{0} q \bar{q}$ states, we would expect the $\pi \pi$ decay mode to identify the $n \bar{n}$ state, whereas $K K$ and $\eta \eta$ final states should be populated by both $n \bar{n}$ and $s \bar{s}$. To illustrate this, in Table $\mathrm{S} 4$ we show the ${ }^{3} \mathrm{P}_{0}$-model predictions for the decays of a pure $s \bar{s} f_{0}(1500)$; a total width of $\Gamma_{t o t}=279 \mathrm{MeV}$ is predicted, with branching fractions of $B_{K K}=76 \%$ and $B_{\eta \eta}=24 \%$.

In contrast to $s \bar{s}$, the flavor-singlet decay amplitudes naively expected for an unmixed glueball should populate both $\pi \pi$ and $K K$ modes. The relative flavor-singlet branching fractions (with phase space removed) are

$$
\begin{array}{r}
B(\underline{\mathbf{1}}) / p . s .\left(\pi \pi: K K: \eta \eta: \eta \eta^{\prime}: \eta^{\prime} \eta^{\prime}\right)= \\
3: 4: 1: 0: 1 .
\end{array}
$$

Three $\mathrm{I}=0$ scalar states are known in this mass region, the $f_{0}(1370), f_{0}(1500)$ and $f_{0}(1710)$; the experimental status of these states was summarized recently by Amsler [57. The branching fraction ratios reported by the Crystal Barrel and WA102 Collaborations for the $f_{0}(1500)$ and $f_{0}(1710)$ are given in Table 3. Neither of the higher-mass states shows the flavor-singlet decay pattern expected for a scalar glueball; instead the $f_{0}(1500)$ strongly favors $\pi \pi$ over $K K$, whereas the $f_{0}(1710)$ favors $K K$ over $\pi \pi$. Since the observed branching fractions of these states do not match the expectations for decays of unmixed states, several studies of $3 \times 3$ mixing models have been carried out in which the scalars are allowed $|n \bar{n}\rangle,|s \bar{s}\rangle$ and $|G\rangle$ components; see for example Refs. [15, 58]. In these studies Amsler and Close [15, 57] concluded that the $f_{0}(1370), f_{0}(1500)$ and $f_{0}(1710)$ are dominantly $n \bar{n}, G$ and $s \bar{s}$ respectively. In contrast, Weingarten et al. [58] prefer the assignments $f_{0}(1500) \approx s \bar{s}$ and $f_{0}(1710) \approx G$.

There is evidence of an additional complication, which is that the intrinsic strong decay amplitudes of the basis states themselves are strongly model- and parameter-dependent. Determination of the state mixing matrix from decay branching fractions, assuming slowly varying decay amplitudes as is done in the mixing models may therefore lead to inaccurate results. One concern is that the ${ }^{3} \mathrm{P}_{0}$-model decay amplitude for $f_{0}^{q \bar{q}} \rightarrow$ PsPs has a node at $\left|\vec{p}_{f}\right|=(3 / \sqrt{2}) \beta \approx 0.8 \mathrm{GeV}$. This is close enough to the physical momenta of final pseudoscalars to invalidate the use of simple relative flavor factors, especially in $f_{0}(1710)$ decays. In addition, Ackleh et al. [5] found that the usually neglected OGE decay amplitude is anomalously large in $f_{0}^{q \bar{q}} \rightarrow \mathrm{PsPs}$, so the ${ }^{3} \mathrm{P}_{0}$ decay amplitude may not be dominant in scalar decays. Finally, there is evidence from LGT of violation of the naive flavor-singlet G-PsPs coupling amplitude often assumed for a pure glue state; see Sexton et al. 61]. 
Since these states may well have important flavor mixing, and the strong decay amplitudes for scalars may have strong momentum dependence, other aspects of these states should be studied for information regarding their Hilbert space decomposition. In particular, radiative transitions may be a more appropriate approach for the identification of the $n \bar{n}$ and $s \bar{s}$ components of these states, since one-photon transitions from $n \bar{n}$ basis states will populate $\gamma \omega$ and $\gamma \rho^{o}$, whereas $s \bar{s}$ will populate $\gamma \phi$ [62]. A simple study of the invariant mass distributions of $\gamma \omega$ and $\gamma \phi$ should tell us a great deal about flavor mixing in the scalar sector.

The two-photon couplings of these states may similarly be effective in identifying their $q \bar{q}$ components, since the $1^{3} \mathrm{P}_{0} n \bar{n}$ scalar is predicted to have a larger $\gamma \gamma$ width than any other $q \bar{q}$ state. An $s \bar{s}$ state should naively have a two-photon width about $2 / 25$ as large as its $\mathrm{I}=0 \mathrm{n} \bar{n}$ partner, whereas a glueball should have a weak $\gamma \gamma$ coupling. (Vector dominance may modify this simple picture, for example if a glueball has a large $\rho \rho$ coupling.) The recent strong L3 limit on the $\gamma \gamma$ partial width of the $f_{0}(1500)$ 39 may constitute evidence that the $n \bar{n}$ component of this state is rather small. In contrast, the $f_{0}(1710)$ may have been seen in $\gamma \gamma \rightarrow K_{S} K_{S}$ by L3 [63, 64] and Belle [65]. (There is some disagreement between these experiments; L3 favors dominance of $K_{S} K_{S}$ by $\mathrm{J}=2$, whereas Belle favors $J=0$.) Future experimental studies of two-photon widths should prove very interesting as tests of the nature of the scalar states.

\subsection{4 $h_{1}(1386)$}

The $h_{1}(1386)$ has been reported by only two experiments, LASS [51] and Crystal Ball [66]. It is nonetheless a convincing candidate for the $s \bar{s}$ partner of the $1^{1} \mathrm{P}_{1}$ states $h_{1}(1170)$ and $b_{1}(1230)$, in view of its mass and dominant decay to $K K^{*}$. ( $K K^{*}$ is the only open-flavor decay channel available to a $1^{+-} s \bar{s}$ state at this mass.) The total width of $91 \pm 30 \mathrm{MeV}$ reported by the PDG is problematic because the state lies at $K K^{*}$ threshold, so the $K K^{*}$ mass distribution and effective width will not be well described by a Breit-Wigner form. We may compare the reported total width with expectations for a $1^{1} \mathrm{P}_{1} s \bar{s}$ state in a qualitative manner by varying the assumed $h_{1}$ mass. As we increase the mass from 1390 to $1440 \mathrm{MeV}$ (by roughly $\Gamma_{\text {expt }} / 2$ ), the predicted width varies from 0 to $160 \mathrm{MeV}$ (Fig.1). Since this range is qualitatively similar to the experimental $91 \pm 30 \mathrm{MeV}$, the assignment of this state to $1^{1} \mathrm{P}_{1} s \bar{s}$ appears plausible.

Theoretical modeling of the $s \bar{s}$ state and $K K^{*}$ continuum as a coupled-channel problem, including the effect of the nonzero $K^{*}$ width, should allow predictions of the expected $K K^{*}$ distributions for both resonant $\left(s \bar{s} \leftrightarrow K K^{*}\right)$ and non-resonant ( $K K^{*}$ threshold enhancement) descriptions of the $h_{1}(1386)$.

\section{$3.6 \quad 2 \mathrm{P}$ States}

\subsection{1 $f_{2}(2000)$}

The $2^{3} \mathrm{P}_{2} s \bar{s}$ tensor $f_{2}(2000)$ is predicted to be a broad state, with a total width near $400 \mathrm{MeV}$, decaying dominantly to $K K^{*}$ and $K^{*} K^{*}$. (See Table $\mathrm{S} 7$ for decays of $2^{3} \mathrm{P}_{\mathrm{J}}$ states.) The $K^{*} K^{*}$ mode has three nonzero amplitudes, and the ${ }^{3} \mathrm{P}_{0}$ model anticipates nontrivial relative strengths; the dominant S- and D-wave spin-quintet amplitudes are predicted to be comparable, ${ }^{5} \mathrm{D}_{2} /{ }^{5} \mathrm{~S}_{2}=-0.59$, and the quintet and singlet $\mathrm{D}$-wave amplitudes are in the ratio ${ }^{5} \mathrm{D}_{2} /{ }^{1} \mathrm{D}_{2}=-\sqrt{7}$. (The spin-triplet amplitude ${ }^{3} \mathrm{D}_{2}$ is identically zero due to C-parity.)

Unfortunately there are no $s \bar{s}$-signature modes open to this state, given our assumed mass of $2000 \mathrm{MeV}$. However for this broad state we would expect to observe some coupling to the $s \bar{s}$-signature mode $\phi \phi$ above threshold, and the intrinsic strength of this mode is quite large; at a mass of $2100 \mathrm{MeV}$, 
the theoretical partial width is $\Gamma_{\phi \phi}=143 \mathrm{MeV}$. The ${ }^{5} \mathrm{~S}_{2}$ amplitude is dominant in $f_{2}(2100) \rightarrow \phi \phi$, however the D-waves should be observable $\left({ }^{5} \mathrm{D}_{2} /{ }^{5} \mathrm{~S}_{2}=-0.12\right.$ given this mass $)$ and have the same characteristic pattern as in $K^{*} K^{*},{ }^{1} \mathrm{D}_{2}:{ }^{5} \mathrm{D}_{2}=1:-\sqrt{7} \cdot\left({ }^{3} \mathrm{D}_{2}\right.$ is forbidden to $\phi \phi$ states by Bose symmetry.)

Since the experimental spectrum at this high mass is poorly established, it is not possible to identify clear experimental candidates for this state. There is a LASS report 67 of a resonance in $K^{*} K^{*}$ with a mass and width of $\mathrm{M}=1950 \pm 15 \mathrm{MeV}$ and $\Gamma=250 \pm 50 \mathrm{MeV}$, which might be this $2^{3} \mathrm{P}_{2} s \bar{s}$ state. However, little is known about this state at present; possible $\mathrm{J}^{\mathrm{PC}}$ quantum numbers include $1^{+-}$and $2^{-+}$in addition to $2^{++}$, and the isospin has not yet been determined.

In view of the predicted strong coupling of the $2^{3} \mathrm{P}_{2} s \bar{s}$ state to $\phi \phi$, the signals reported in this channel in previous glueball searches should be assessed as possibly due to this state. (The other $2^{++}$ $s \bar{s}$ state expected near this mass, the $1^{3} \mathrm{~F}_{2} f_{2}(2200)$, is predicted to have a very weak $\phi \phi$ coupling.) The reaction $\pi^{-} p \rightarrow \phi \phi n$ was studied at BNL [68], and evidence for three $2^{++}$states at masses of $2011_{-76}^{+62}, 2297 \pm 28$ and $2339 \pm 55 \mathrm{MeV}$ was reported. The first of these BNL states is an obvious candidate for the theoretical $2^{3} \mathrm{P}_{2} s \bar{s} f_{2}(2000)$. The experimental $f_{2}(2011)$ was found to have a $\phi \phi$ partial width decomposition of $B\left({ }^{5} \mathrm{~S}_{2}\right)=98_{-3}^{+1} \%, B\left({ }^{5} \mathrm{D}_{2}\right)=0^{+1} \%$ and $B\left({ }^{1} \mathrm{D}_{2}\right)=2_{-1}^{+2} \%$ 68]. The S-wave is clearly dominant as predicted for $2^{3} \mathrm{P}_{2} s \bar{s}$, however this is unsurprising given the lack of phase space. One might test a $2^{3} \mathrm{P}_{2} s \bar{s}$ assignment for the $f_{2}(2011)$ by searching for this state in $K^{*} K^{*}$ and $K K^{*}$ final states.

\subsubsection{The unobserved $f_{1}(1950)$}

The $2^{3} \mathrm{P}_{1} s \bar{s}$ state is predicted to be moderately broad, with $\Gamma_{\text {tot }} \approx 300 \mathrm{MeV}$. It may be most easily identified in the $K K^{*}$ mode, in which it has a very characteristic dominance of D-wave $K K^{*}$ final states over S-wave. Evidence for this unusual amplitude ratio has been reported for the $a_{1}(1700)$ [69, 70], which is a candidate $2^{3} \mathrm{P}_{1} \mathrm{I}=1$ partner of the $f_{1}(1950)$.

A nonexotic $n \bar{n}$-hybrid with $\mathrm{J}^{\mathrm{PC}}=1^{++}$is predicted at a similar mass in the flux-tube model [43, 44]. The Isgur-Paton flux-tube decay model predicts that this will be a very broad state [45], however a ${ }^{3} \mathrm{~S}_{1}$ variant of the flux-tube decay model studied by Page, Swanson and Szczepaniak [23] suggests that this hybrid might be rather narrow. In the latter case overpopulation of the $1^{++}$sector of the quark model near this mass might easily be confirmed. The hybrid, unlike the $2^{3} \mathrm{P}_{1} s \bar{s}$ state, is predicted by Page et al. [23] to have a dominant S-wave amplitude in its $K K^{*}$ decay mode.

\subsubsection{The unobserved $f_{0}(2000)$}

The $2^{3} \mathrm{P}_{0} s \bar{s} f_{0}(2000)$ is predicted to be very broad, with a total width of $\Gamma_{t o t} \approx 800 \mathrm{MeV}$. This is the largest total width predicted for any of the states considered in this paper. The dominant mode is expected to be $K K_{1}(1273)$; this mode is also predicted to dominate the decays of another broad state, the $1^{3} \mathrm{D}_{1} s \bar{s} \phi(1850)$. The $f_{0}(2000)$ theoretically has sufficiently strong couplings to $K K$ and $K^{*} K^{*}$ to be identified in those channels, especially if the coupling to $K K_{1}(1273)$ and resulting very large total width are overestimated by the ${ }^{3} \mathrm{P}_{0}$ decay model. Unfortunately there are no characteristic $s \bar{s}$-signature modes open to this state, with the possible exception of the very problematical channel $\eta \eta_{s}(1415)$.

\subsubsection{The unobserved $h_{1}(1850)$}

Unlike the other $2 \mathrm{P} s \bar{s}$ states, the $2{ }^{1} \mathrm{P}_{1} h_{1}(1850)$ is predicted to be moderately narrow, with $\Gamma_{\text {tot }}=193$ $\mathrm{MeV}$. (See Table S8.) Only four open-flavor modes are accessible to the $h_{1}(1850)$, and of these one 
( $K K_{1}(1273)$ ) is predicted to be numerically unimportant. The modes $K K^{*}$ and $K^{*} K^{*}$ are largest, but the relatively large branching fraction predicted to the $s \bar{s}$-signature mode $\eta \phi\left(B_{\eta \phi} \approx 15 \%\right)$ and the smaller backgrounds expected in this channel suggest that $\eta \phi$ should be ideal for identifying the $h_{1}(1850)$.

The large photoproduction cross section reported for the $1^{1} \mathrm{P}_{1} h_{1}(1170)$ [71] makes the $2^{1} \mathrm{P}_{1}$ $h_{1}(1850)$ an attractive target for diffractive photoproduction. Since the flux-tube model predicts nonexotic hybrids with these quantum numbers nearby in mass [43, 44], it will be important to identify this state as a "background" quarkonium resonance.

\section{7 $1 \mathrm{D}$ States}

\subsection{1 $\phi_{3}(1854)$}

The $\phi_{3}(1854)$ was first reported in $K^{-} p \rightarrow \phi_{3} \Lambda$ in a 1981 CERN bubble-chamber experiment [72]. It was reported in $K K$ and $K K^{*}$, with a total width of $50-120 \mathrm{MeV}$ and a relative branching fraction of $B_{K K^{*} / K K}=0.8 \pm 0.4$. Subsequently in 1982 the Omega Spectrometer Collaboration 73 at CERN observed the $\phi_{3}$ in $K^{+} K^{-}$, and reported a mass and width of $\mathrm{M}=1850-1900 \mathrm{MeV}$ and $\Gamma=110-250$ $\mathrm{MeV}$. More recently the LASS Collaboration [74] observed the $\phi_{3}$ in $K^{+} K^{-}$and $K_{S} K^{ \pm} \pi^{\mp}$, and in several fits found masses and widths of $\mathrm{M} \approx 1855 \mathrm{MeV}$ and $\Gamma \approx 60 \pm 30 \mathrm{MeV}$. The PDG gives averaged masses and widths of $\mathrm{M}=1854 \pm 7 \mathrm{MeV}$ and $\Gamma=87_{-23}^{+28} \mathrm{MeV}$ 近. A branching ratio of $B_{K K^{*} / K K}=0.55_{-0.45}^{+0.85}$ was quoted by LASS.

In the ${ }^{3} \mathrm{P}_{0}$ model with our parameters we predict a total $\phi_{3}(1854)$ width of $104 \mathrm{MeV}$ and a $B_{K K^{*} / K K}$ branching fraction of 0.52 (Table S9), consistent with experimental estimates. We also predict a large $K^{*} K^{*}$ mode, with a relative $B_{K^{*} K^{*}} / B_{K K}$ branching fraction of 0.70 . The $K^{*} K^{*}$ mode is interesting in that four independent amplitudes are allowed; the ${ }^{3} \mathrm{P}_{0}$ model predicts the ${ }^{5} \mathrm{P}_{3}$ $K^{*} K^{*}$ amplitude to be dominant and ${ }^{5} \mathrm{H}_{3}$ to be zero. (Decay to the ${ }^{3} \mathrm{~F}_{3} K^{*} K^{*}$ state is forbidden by C-parity.)

\subsubsection{The unobserved $\phi_{2}(1850)$}

The identification of this state would be very interesting, as no $2^{--}$states are known at present. The $\phi_{2}(1850)$ is attractive experimentally because the mass of the 1D $s \bar{s}$ multiplet is well established by the $\phi_{3}(1854)$, and the total width is predicted to be relatively small, $\Gamma_{t o t}=214 \mathrm{MeV}$. Only two decay modes are predicted to have large branching fractions, $K K^{*}$ and $\eta \phi$. The latter is a very attractive $s \bar{s}$-signature mode, which we expect to coupling strongly only to states with large $s \bar{s}$ components.

The $\phi_{2}(1850)$ can be diffractively photoproduced, although the strength of the $2^{--}$photoproduction amplitude is not known. The dominant $K K^{*}$ and $\eta \phi$ final states will allow tests of the ${ }^{3} \mathrm{P}_{0}$ model, since these modes are predicted to have significant ${ }^{3} \mathrm{P}_{2}$ and ${ }^{3} \mathrm{~F}_{2}$ amplitudes. We predict ${ }^{3} \mathrm{~F}_{2} /{ }^{3} \mathrm{P}_{2}$ amplitude ratios of -0.34 for $K K^{*}$ and -0.19 for $\eta \phi$ (Table S9). A measurement of this ratio in either decay would provide an important test of the ${ }^{3} \mathrm{P}_{0}$ model in a new angular channel; the existing accurate amplitude ratio tests have only considered decays of $\mathrm{L}=1$ mesons.

\subsubsection{The unobserved $\phi(1850)$}

The $1^{3} \mathrm{D}_{1} s \bar{s} \phi(1850)$ is predicted to be a very broad resonance, $\Gamma_{t o t} \approx 650 \mathrm{MeV}$, due to a very large Swave coupling to the $K K_{1}(1273)$ decay channel. Although this appears discouraging experimentally, one should note that there has been no experimental confirmation of the theoretically very large $1^{3} \mathrm{D}_{1} \rightarrow 1^{1} \mathrm{~S}_{0}+1^{3} \mathrm{P}_{1}$ and $1^{3} \mathrm{D}_{1} \rightarrow 1^{1} \mathrm{~S}_{0}+1^{1} \mathrm{P}_{1}$ decay amplitudes in any flavor channel; if the ${ }^{3} \mathrm{P}_{0}$ 
model has significantly overestimated these amplitudes, the $\phi(1850)$ might be considerably narrower. Rather smaller couplings to $K K$ and $K K^{*}$ are predicted, with branching fractions of $\approx 10 \%$. The branching fraction to the $s \bar{s}$-signature mode $\eta \phi$ is expected to be $\approx 5 \%$.

The very strong coupling predicted to $K K_{1}(1273)$ may be tested independently, assuming that the $\omega(1649)$ and $\rho(1700)$ are the $\mathrm{I}=0$ and $\mathrm{I}=11^{3} \mathrm{D}_{1} n \bar{n}$ partners of the hypothetical $\phi(1850)$. These $n \bar{n}$ states are predicted to have analogously large decay amplitudes in $\omega(1649) \rightarrow \pi b_{1}$ and $\rho(1649) \rightarrow$ $\pi a_{1}, \pi h_{1}$, which will presumably be studied in $e^{+} e^{-}$at VEPP and DAPHNE.

Determination of the excited vector spectrum is of interest in part because the flux-tube model anticipates vector hybrids [43, 44], which the existence of the $\pi_{1}(1600)$ suggests may be in this mass region. The vector $s \bar{s}$-hybrid is predicted to have a rather smaller total width than this $s \bar{s}$ quark model state [23, 45].

\subsection{4 $\eta_{2}(1850)$}

Assuming a mass of $1850 \mathrm{MeV}$ for the $1^{1} \mathrm{D}_{2} s \bar{s}$ state, only three open-flavor modes are accessible given our nominal masses, $K K^{*}, K^{*} K^{*}$ and $K K_{1}(1273)$ (see Table S10). $K K^{*}$ is predicted to be dominant, with a branching fraction of $\approx 90 \%$ and a rather large $\mathrm{F}$-wave component, ${ }^{3} \mathrm{~F}_{2} /{ }^{3} \mathrm{P}_{2}=+0.52$. The remaining decays are expected to populate $K^{*} K^{*}$ almost exclusively. The predicted total width is rather small, $\Gamma_{\text {tot }}=129 \mathrm{MeV}$, due to few open modes, limited phase space, and the centrifical barriers present in all cases.

Experimentally there are two known resonances with these quantum numbers, the $\eta_{2}(1617)$ and $\eta_{2}(1842)$. In view of the mass of the $\mathrm{I}=1 \pi_{2}(1670)$, these two $\eta_{2}$ states would appear to be $n \bar{n}$ and $s \bar{s} 1^{1} \mathrm{D}_{2}$ candidates, although the total width of the $\eta_{2}(1842), \Gamma_{\text {expt }}=225 \pm 14 \mathrm{MeV}$, is somewhat larger than our estimate for the $1^{1} \mathrm{D}_{2} s \bar{s}$ state. Although LASS did not claim an isoscalar $2^{-+}$ resonance, their data suggest an enhancement at $1.8-1.9 \mathrm{GeV}$ in the $2^{-+} K_{S}^{0} K^{ \pm} \pi^{\mp}$ partial wave in $K^{-} p \rightarrow K_{S}^{0} K^{ \pm} \pi^{\mp} \Lambda$ (see Fig.2e of Ref. [51]). Since this production process enhances $s \bar{s}$ relative to $n \bar{n}$, LASS may have evidence that the higher-mass $\eta_{2}$ is dominantly an $s \bar{s}$ state. The $K_{S}^{0} K^{ \pm} \pi^{\mp}$ final state can arise from $K K^{*}$, which we predict to be the principal decay mode of the $1^{1} \mathrm{D}_{2} s \bar{s}$ state.

There are problems with unmixed $n \bar{n}$ and $s \bar{s} 2^{-+}$assignments. The $\eta_{2}(1842)$ has only been reported in $4 \pi$ and $\eta \pi \pi$ modes, which are inaccessible to pure $s \bar{s}$ states in the ${ }^{3} \mathrm{P}_{0}$ decay model. Both $\eta_{2}$ states were reported in double diffraction to $\pi a_{2}$ by WA102, with comparable strengths (see Fig.3e of Ref. [22]), which suggests important $n \bar{n} \leftrightarrow s \bar{s}$ mixing if both states are indeed $q \bar{q}$. The lighter $\eta_{2}(1617)$ has been reported by WA102 in both $\pi a_{2}$ and $K K \pi$ [48, and the experimental ratio $B_{K K \pi / \pi a_{2}}=0.07 \pm 0.03$ is not far from our prediction of 0.14 for a pure $n \bar{n} 1^{1} \mathrm{D}_{2}$ state 21]. This suggests that flavor mixing in the $\eta_{2}$ system is not very large, contrary to what is implied by the relative $\pi a_{2}$ strengths.

We can test the possibility of significant $n \bar{n} \leftrightarrow s \bar{s}$ flavor mixing in the $\eta_{2}$ system by generalizing our ${ }^{3} \mathrm{P}_{0}$ decay calculations to mixed initial states

$$
\left|\eta_{2}(1617)\right\rangle=\cos (\phi)|n \bar{n}\rangle_{0}-\sin (\phi)|s \bar{s}\rangle
$$

and

$$
\left|\eta_{2}(1842)\right\rangle=\sin (\phi)|n \bar{n}\rangle_{0}+\cos (\phi)|s \bar{s}\rangle
$$

where we have assigned these the PDG experimental masses. The resulting decay amplitudes and partial widths are given in Tables S11 and S12. The partial widths of the $\eta_{2}(1842)$ to the three important modes $\pi a_{2}, K K^{*}$ and $\rho \rho$ are shown in Fig.2 as functions of the flavor mixing angle $\phi$. 


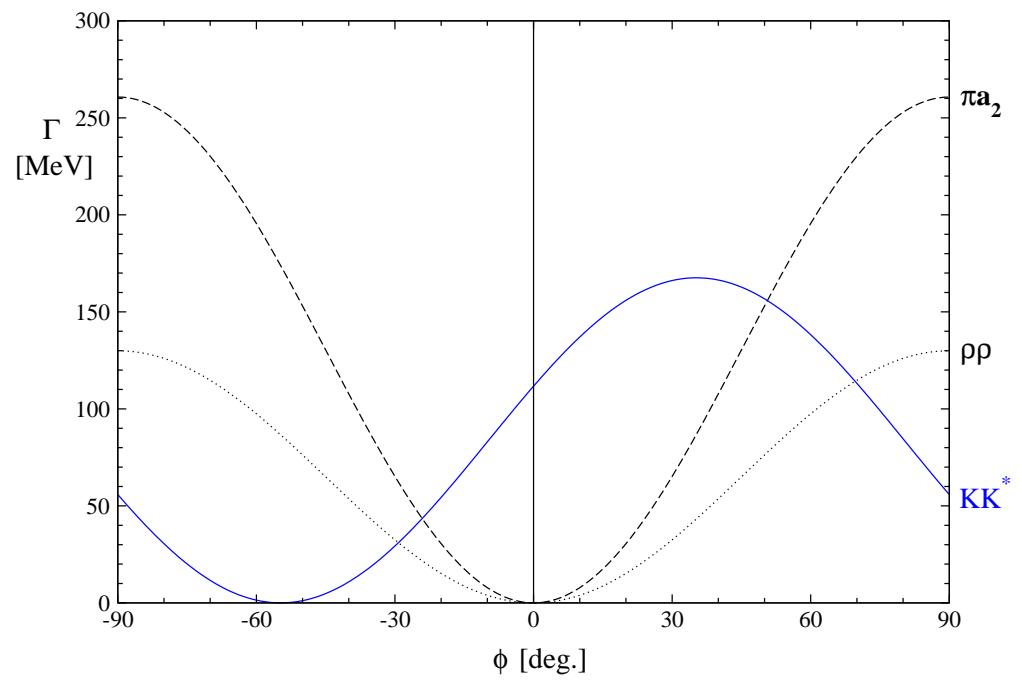

Figure 2. Theoretical widths of the three leading modes of a flavor-mixed $1^{1} \mathrm{D}_{2} \eta_{2}(1842)$.

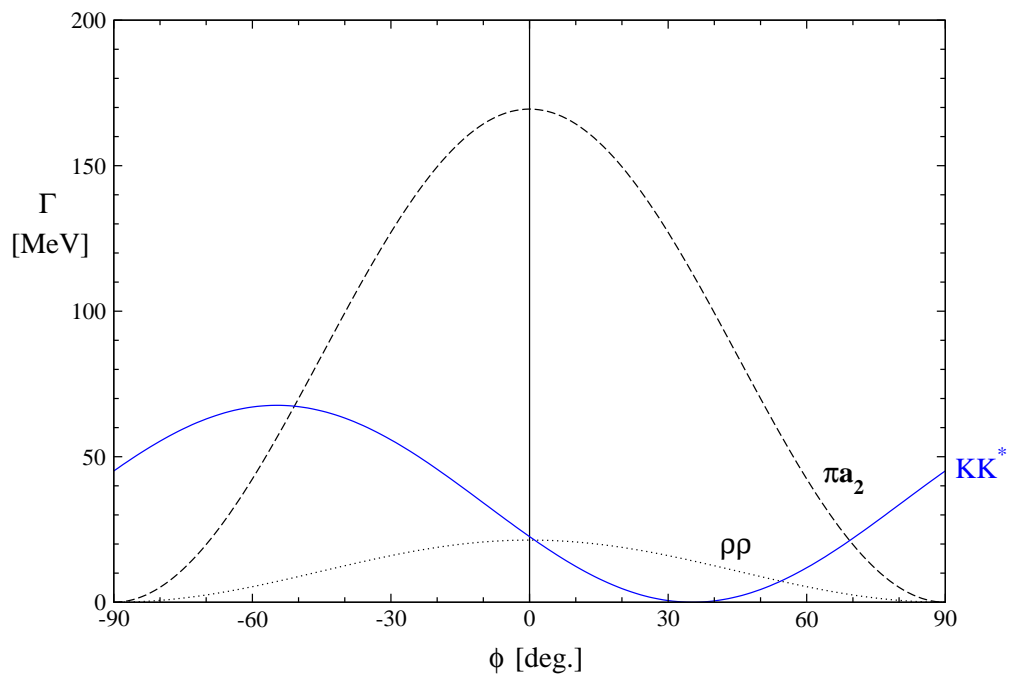

Figure 3. Theoretical widths of the orthogonal partner $1^{1} \mathrm{D}_{2} \eta_{2}(1617)$. 
Evidently, large couplings to $\pi a_{2}$ and $\rho \rho$ follow from moderate mixing, which could explain the WA102 observation of the $\eta_{2}(1842)$ in $\eta \pi \pi$ and $4 \pi$. Since the ratio $B_{K K^{*}} / B_{\pi a_{2}}$ is strongly dependent on the flavor mixing angle $\phi$, this ratio may be useful in determining $\phi$ if the $\eta_{2}(1842)$ is indeed a quark model state.

At present however the assignment of the $\eta_{2}(1617)$ and $\eta_{2}(1842)$ to a mixed-flavor quark model pair appears implausible, due to the $K K^{*}$ final state. The dominant decay modes of an orthogonal partner state $\eta_{2}(1617)$ are shown in Fig. 3 and given in Table S12. The facts that the $\eta_{2}(1617) \rightarrow K K^{*}$ branching fraction observed by WA102 is rather small (see Fig.2e of Ref. 48), $B_{\eta_{2}(1617) \rightarrow K K \pi / \pi a_{2}}=$ $0.07 \pm 0.03$, and that there is no indication of the $\eta_{2}(1842)$ in this data, argues against assigning both reported states to an $n \bar{n} \leftrightarrow s \bar{s}$ mixed pair; our Fig.2 and Fig.3 show that there should be a fairly large $K K^{*}$ mode evident in the combined $\eta_{2}(1617)$ and $\eta_{2}(1842)$ signals, whatever the mixing angle $\phi$. Only the quite weak $\eta_{2}(1617) \rightarrow K K^{*}$ transition is evident.

An alternative possibility is that the higher-mass WA102 state $\eta_{2}(1842)$ is an $n \bar{n}$-hybrid rather than a mixed $n \bar{n} \leftrightarrow s \bar{s}$ quark model state, and the PsV coupling of the hybrid is rather small; for some reason the PsT mode $\pi a_{2}$ is preferred. Assuming the hybrid assignment, we would expect to find evidence of an $\mathrm{I}=12^{-+}$partner hybrid at a similar mass. There have been several reports of possible $\pi_{2}$ states in this mass region, notably a D-wave $\pi f_{2}$ signal reported by ACCMOR in 1981 [75] that peaks near $1850 \mathrm{MeV}$. Several other possible higher-mass $\pi_{2}$ signals are discussed in Ref. 21]. Quite recently a state with these quantum numbers and resonant phase motion was reported by the E852 Collaboration in $\rho^{-} \omega$ [76], with a mass and width of $\mathrm{M}=1890 \pm 10 \pm 26 \mathrm{MeV}$ and $\Gamma=350 \pm 22 \pm 55 \mathrm{MeV}$. This exciting result may imply that a flavor nonet of nonexotic $2^{-+}$ hybrids exists at a mass of $1.8-1.9 \mathrm{GeV}$ (for $n \bar{n}$ flavor), just as anticipated by the flux tube model 443, 44].

\section{$3.8 \quad 1 F$ States}

\subsubsection{F $s \bar{s}$ and the " $\xi(2230)$ " region}

The $1 \mathrm{~F} s \bar{s}$ multiplet has long been of interest because of the Mark III [77] and BES [78] reports of a possible very narrow $\xi(2230)$ in $J / \psi$ radiative decays. This evidence is controversial because DM2 [79] did not see this state, although they had slightly better statistics than Mark III. The JETSET Collaboration studied $K_{S} K_{S}$ 80] and $\phi \phi$ [81 final states in $p \bar{p}$ annihilation at LEAR, and found no evidence for a narrow resonance with the reported $\xi(2230)$ mass and width. The Crystal Barrel Collaboration [82] also saw no evidence for this narrow state in $p \bar{p} \rightarrow \eta \eta$, although the BES results on $p \bar{p}$ and $\eta \eta$ imply that they should have seen a large signal. The most recent experimental developments are extremely strong limits on a narrow $\xi(2230)$ in $\gamma \gamma \rightarrow K_{S} K_{S}$ from L3 [64] and Belle [65],

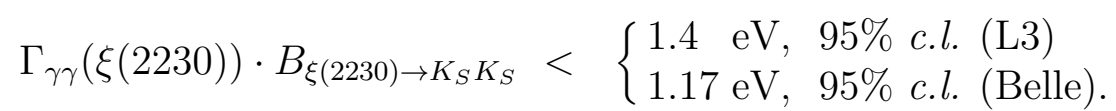

Motivated by the original Mark III results, Godfrey et al. $\llbracket 19$ calculated a subset of $1^{3} \mathrm{~F}_{2}$ and $1^{3} \mathrm{~F}_{4} s \bar{s}$ decay modes (thought to be all the important ones), and found relatively small total widths for these states. (These particular $s \bar{s}$ states were considered because the reported signal had a mass consistent with expectations for the $1 \mathrm{~F} s \bar{s}$ multiplet, and had to have even ${ }^{++}$quantum numbers since it was reported in $K_{S} K_{S}$.) These results suggested that the surprisingly narrow $\xi(2230)$, if real, might simply be a conventional $s \bar{s}$ meson rather than a more unusual state such as a glueball or hybrid. Subsequent work by Blundell and Godfrey [16] greatly modified these conclusions. In the 
$1^{3} \mathrm{~F}_{2} s \bar{s}$ case an orbitally excited mode that had previously been neglected $\left(K K_{1}(1273)\right)$ was found to be dominant, making this $2^{++}$state rather broad; this eliminated the tensor $s \bar{s}$ option for the $\xi(2230)$, provided that the ${ }^{3} \mathrm{P}_{0}$ decay model is reasonably accurate. The $1^{3} \mathrm{~F}_{4} s \bar{s}$ state was confirmed to couple primarily to $K^{*} K^{*}, K K^{*}$ and $K K$ in the Blundell-Godfrey work, although a total width of over $100 \mathrm{MeV}$ was found. This was an order of magnitude larger than the $\xi(2230)$ widths reported by Mark III and BES, so the explanation of the $\xi(2230)$ as a $1 \mathrm{~F} s \bar{s}$ state now appears implausible.

There is experimental evidence of a somewhat wider state in this mass region. A state with a mass and width of $\mathrm{M}=2231 \pm 10 \mathrm{MeV}$ and $\Gamma=133 \pm 50 \mathrm{MeV}$ (with $\mathrm{J}$ undetermined) was reported in $\phi \phi$ by WA67 (CERN SPS) [83], and the LASS Collaboration reported a $4^{++}$resonance with a mass and width of $\mathrm{M}=2209_{-15}^{+17} \pm 10 \mathrm{MeV}$ and $\Gamma=60_{-57}^{+107} \mathrm{MeV}$ in $K^{-} p \rightarrow K^{-} K^{+} \Lambda$ and $K^{-} p \rightarrow K_{S} K_{S} \Lambda$ [84, 855]. Very recently, E173 (Serpukhov) also reported an enhancement in $K_{S} K_{S}$, with $\mathrm{M}=2257 \mathrm{MeV}$ and $\Gamma=56 \mathrm{MeV} 86$.

\subsection{2 $f_{4}(2200)$}

The $f_{4}(2200)$ is predicted to be the narrowest of the $1 \mathrm{~F} s \bar{s}$ states, with an expected total width of about $150 \mathrm{MeV}$ (Table S13).. Our results for this state are quite similar to those found by Blundell and Godfrey [16] in their variant of the ${ }^{3} \mathrm{P}_{0}$ model using Kokoski-Isgur phase space. We also find that the three important modes are $K K, K K^{*}$ and $K^{*} K^{*}$. Our partial widths for these modes are comparable, although the precise values are rather sensitive to kinematics because $K K^{*}$ and $K K$ are G-wave final states, with a resulting threshold behavior of $\left|\vec{p}_{f}\right|^{9}$. The observation of the $f_{4}(2200)$ in both $K K$ and $K^{*} K^{*}$ would be interesting in part because of the rather inaccurate prediction of the SU(3) partner decay $f_{4}(2040) \rightarrow \pi \pi$ [2]] (which may be due to this strong $\left|\vec{p}_{f}\right|^{9}$ momentum dependence) and the lack of information regarding $f_{4}(2040) \rightarrow \rho \rho$, which is predicted to have a large branching fraction. There are also (relatively weak) analogous $\phi \phi$ and $\eta \eta$ modes, which measure the same decay amplitudes at different momenta and thus would provide useful information. The multiamplitude $\mathrm{VV}$ mode $K^{*} K^{*}$ is predicted unsurprisingly to be dominated by the lowest- $\mathrm{L}$ amplitude, ${ }^{5} \mathrm{D}_{4}$. Nonetheless an experimental study of the three higher-L amplitudes predicted to be weak or zero would provide an interesting test of the ${ }^{3} \mathrm{P}_{0}$ model.

Identification of the $1^{3} \mathrm{~F}_{4} s \bar{s}$ and a determination of its decay parameters would be an important contribution to our understanding of this historically controversial region of the spectrum. The broader experimental states in the $2200 \mathrm{MeV}$ region, which are discussed at the end of the previous section, are possible candidates for the $f_{4}(2200) 1^{3} \mathrm{~F}_{4} s \bar{s}$ state.

\subsubsection{The unobserved $f_{3}(2200)$}

The $f_{3}(2200) 1^{3} \mathrm{~F}_{3} s \bar{s}$ state is predicted to have a total width of about $300 \mathrm{MeV}$, and to decay dominantly to $K K_{2}^{*}(1429)$, with a branching fraction of $\approx 40 \%$. (The VV and PsV modes have an $\mathrm{L}=2$ barrier, whereas the $K K_{2}^{*}(1429)$ mode is dominantly $\mathrm{P}$-wave.) Next in importance is $K K^{*}$, with a branching fraction of $\approx 25 \%$ and a ${ }^{5} \mathrm{G}_{3} /{ }^{5} \mathrm{D}_{3}$ amplitude ratio of -0.62 . The branching fractions to $K^{*} K^{*}, K K_{1}(1273), K^{*} K_{1}(1273)$ and the unusual mode $\eta f_{2}^{\prime}(1525)$ are each $\approx 5-10 \%$. The $K^{*} K^{*}$ channel has two allowed amplitudes, and the ${ }^{3} \mathrm{P}_{0}$ model predicts the ${ }^{5} \mathrm{G}_{3} /{ }^{5} \mathrm{D}_{3}$ amplitude ratio to be +0.51 . Interesting measurements here include the $\mathrm{G} / \mathrm{D}$ ratio in $K K^{*}$, a test of the predicted dominance of $K K_{2}^{*}(1429)$, and the presence of this state in $\eta f_{2}^{\prime}(1525)$, which in the decay model is due to an $(s \bar{s}) \rightarrow(s \bar{s})+(s \bar{s})$ transition. 


\subsubsection{The unobserved $f_{2}(2200)$}

The $f_{2}(2200)$ is predicted to have a very large decay coupling to $K K_{1}(1273)$, which would make this a rather broad state; the expected total width is $425 \mathrm{MeV}$, with $B_{K K_{1}(1273)} \approx 60 \%$. The other decay modes of this state have theoretical branching fractions of $<10 \%$ and are not especially characteristic of $s \bar{s}$ states. It may be possible to identify the $f_{2}(2200)$ in $K K$ or $K K^{*}$, or perhaps in the $\eta \eta$ or $\eta \eta^{\prime}$ modes.

One might hope to identify $s \bar{s}$ states in $\phi \phi$, which has previously been studied experimentally in searches for glueball resonances, notably in $\pi^{-} p \rightarrow \phi \phi n$ at BNL [68]. Three tensor states were reported in $\phi \phi$ at BNL, and the two near our assumed $1 \mathrm{~F} s \bar{s}$ mass of $2200 \mathrm{MeV}$ were at $2297 \pm 28$ and $2339 \pm 55 \mathrm{MeV}$. These a priori appear to be natural candidates for the $1^{3} \mathrm{~F}_{2} s \bar{s}$ quark model state, and the reported $f_{2}(2297) \rightarrow \phi \phi$ strengths in different $\phi \phi$ waves are similar to the pattern predicted for $1^{3} \mathrm{~F}_{2}(s \bar{s}) \rightarrow \phi \phi$; Etkin et al. 68 reported $B\left({ }^{5} \mathrm{~S}_{2}\right)=6{ }_{-5}^{+15} \%, B\left({ }^{1} \mathrm{D}_{2}\right)=69_{-27}^{+16} \%$ and $B\left({ }^{5} \mathrm{D}_{2}\right)=25_{-14}^{+18} \%$, whereas for a $1^{3} \mathrm{~F}_{2} s \bar{s} f_{2}(2300)$ (note the increased mass) we predict $B\left({ }^{5} \mathrm{~S}_{2}\right)=0 \%$, $B\left({ }^{1} \mathrm{D}_{2}\right)=49 \%, B\left({ }^{5} \mathrm{D}_{2}\right)=28 \%$ and $B\left({ }^{5} \mathrm{G}_{2}\right)=23 \%$. The theoretical ratio of $\mathrm{D}$-wave partial widths in $1^{3} \mathrm{~F}_{2}(s \bar{s}) \rightarrow \phi \phi$ is $B\left({ }^{1} \mathrm{D}_{2}\right) / B\left({ }^{5} \mathrm{D}_{2}\right)=7 / 4$.

In the ${ }^{3} \mathrm{P}_{0}$ model however the $1^{3} \mathrm{~F}_{2}(s \bar{s}) \rightarrow \phi \phi$ branching fraction is predicted to be very small $(0.5 \%$ for $\mathrm{M}=2200 \mathrm{MeV})$, and unless this small branching fraction is confirmed, identification of any of the resonances seen in $\phi \phi$ with the $1^{3} \mathrm{~F}_{2} s \bar{s}$ is questionable. Of course one should not eliminate the possibility that this tiny decay coupling may simply be an inaccurate prediction of the ${ }^{3} \mathrm{P}_{0}$ decay model; this predicted small coupling should be checked against the VV decays of other members of the $1^{3} \mathrm{~F}_{2}$ flavor nonet, once these are identified.

\subsubsection{The unobserved $h_{3}(2200)$}

The spin-singlet $h_{3}(2200)$ (Table S4) is predicted to have a moderate total width of $\Gamma_{\text {tot }} \approx 250 \mathrm{MeV}$. This $\mathrm{C}=(-)$ state can be diffractively photoproduced, and the production amplitudes of a higherL state may provide interesting information about the nature of diffraction. The decay modes $K K_{2}^{*}(1429)$ and $K K^{*}$ are predicted to be dominant. The $s \bar{s}$-signature mode $\eta \phi$ is more attractive experimentally; the $h_{3}(2200)$ is predicted to have a rather large, ca. $10 \%$ branching fraction to $\eta \phi$, with comparable strengths in $\mathrm{D}$ - and $\mathrm{G}$-waves. The ratios we find for these amplitudes are ${ }^{3} \mathrm{G}_{3} /{ }^{3} \mathrm{D}_{3}=+0.59$ for $\eta \phi$ and +0.83 for the partner open-strangeness mode $K K^{*}$. 


\section{Kaonia}

\subsection{General aspects}

The kaon sector is interesting for several reasons. One notable feature is that the usual kaon and antikaon states do not have diagonal C-parity, so there are no $\mathrm{J}^{\mathrm{PC}}$-exotics in the kaon spectrum. The kaon-flavor analogues of $n \bar{n}$ and $s \bar{s} \mathrm{~J}^{\mathrm{PC}}$-exotic hybrids should instead appear as a rich overpopulation of states in the conventional excited kaon spectrum.

A detailed comparison between the kaon and $\mathrm{I}=1 n \bar{n}$ spectra may therefore be useful for the identification of hybrids using overpopulation. For this comparison one should specialize to $\mathrm{J}^{\mathrm{P}}=$ $0^{-}, 0^{+}, 1^{-}, 2^{+}, 3^{-} \ldots$, for which one C-partner is exotic. For example, the $\mathrm{J}^{\mathrm{P}}=1^{-}$kaon spectrum will have an overpopulation of states relative to $\mathrm{J}^{\mathrm{PC}}=1^{--} \mathrm{I}=1 n \bar{n}$, due to the presence of both $\mathrm{J}^{\mathrm{PC}}=1^{--}$quarkonium and $\mathrm{J}^{\mathrm{PC}}=1^{-+}$hybrid basis states in the $\mathrm{J}^{\mathrm{P}}=1^{-}$kaon mixing problem.

Not only will there be "too many states" in a given kaon $\mathrm{J}^{\mathrm{P}}$ sector relative to $\mathrm{I}=1 n \bar{n}$, we also anticipate irregularities between the kaon and $\mathrm{I}=1 n \bar{n}$ spectra, due to mass shifts from kaon mixing with $\mathrm{J}^{\mathrm{PC}}$-exotic hybrid basis states that cannot mix in the $\mathrm{I}=1 n \bar{n}$ problem. The anomalously low mass of the $K^{*}(1414)$ relative to the $\rho(1465)$ may be an example of this effect.

The absence of C-parity also implies that the physical $\mathrm{J}^{\mathrm{P}}$ kaon states are admixtures of spin-singlet and spin-triplet $q \bar{q}$ basis states with different $\mathrm{C}$ for $\mathrm{J}^{\mathrm{P}}=1^{+}, 2^{-}, 3^{+} \ldots$, unlike their neutral $\mathrm{I}=1 n \bar{n}$ partners. The $K_{1}$ system is a familiar example of this mixing; the physical $K_{1}(1273)$ and $K_{1}(1402)$ are strongly mixed linear combinations of $\left|1^{1} \mathrm{P}_{1}\right\rangle$ and $\left|1^{3} \mathrm{P}_{1}\right\rangle$ basis states. The precise mechanism of this mixing of different $S_{q \bar{q}}$ states is an interesting open question in the kaon system. Mixing has been attributed to coupling through decay channels (originally by Lipkin [87]) as well as to $q \bar{q}$ spin nonconservation in the OGE spin-orbit interaction (because $m_{s} \neq m_{u, d}$ ), although this effect does not appear large enough to explain the observed 1P mixing angle 88. The dominant mechanism of singlet-triplet mixing has evidently not yet been definitively established, and can presumably be clarified through additional theoretical studies and measurements of the corresponding mixing angles in the $2 \mathrm{P}, 1 \mathrm{D}$ and $1 \mathrm{~F}$ systems. Experience with the $K_{1}$ system suggests that strong decays of the higher-mass states will allow determination of these mixing angles; to assist in this exercise we give the mixing-angle dependence of strong partial widths and decay amplitudes for general mixed states in the decay tables.

The fact that the kaon sector has no valence annihilation may also make it useful for the identification of large mixing effects between $\mathrm{I}=0 n \bar{n}, s \bar{s}$ and glueball basis states. A comparison of the spectra in these different flavor sectors may show irregularities where valence annihilation is important, as may be the case in the scalar sector.

In summary, a comparison between the kaon and $\mathrm{I}=1 n \bar{n}$ spectra should provide evidence for hybrids through kaon overpopulation, and a comparison with the $\mathrm{I}=0 n \bar{n}$ and $s \bar{s}$ spectra may provide evidence of $q \bar{q}$-glueball mixing. Establishing the spectrum of excited kaon states may thus be important for searches for both types of gluonic hadrons expected in the meson spectrum. A determination of singlet-triplet mixing angles in higher-mass kaon states through measurements of branching fractions and decay amplitudes is also an interesting experimental exercise, since these angles have not yet been determined except in the light $K_{1}$ sector, and the mechanism that drives this mixing is not yet understood.

Experimentally there are few plans to study the excited kaon spectrum with improved statistics. This is unfortunate in view of the importance of the kaon spectrum for studies of overpopulation, mixing and valence annihilation effects. Hadronic reactions such as $K^{ \pm} p \rightarrow(K n \pi)^{ \pm} p$ could be explored with a medium-energy RF-separated kaon beam, as is now under construction at Serpukhov. 
This would also be possible at JHF, although this is not part of their current physics program. (At lower beam energies a $K^{+}$beam may be preferable to the usual $K^{-}$, to avoid a large background of schannel strange baryon resonances.) Other possibilities include photoproduction and $e^{+} e^{-}$facilities, which could study higher-mass kaon spectra through the sequential decays of initial $s \bar{s}$ states, and $p \bar{p}$ annihilation in flight at GSI [89]. In $p \bar{p}$ annihilation one may extract higher-mass kaon resonances for example from partial wave analyses of $p \bar{p} \rightarrow K+(K n \pi)$.

Finally, the high-statistics studies of heavy-quark physics and $\mathrm{CP}$ violation at $\mathrm{D}$ and $\mathrm{B}$ factories can contribute to the study of excited kaon spectroscopy, through the identification of resonances in final states with a kaon. The excited kaons already reported in heavy-quark nonleptonic weak decays are the $K_{1}(1273), K_{1}(1402), K_{0}(1412)$ and $K^{*}(1717)$ (in D decays, typically at the $1 \%$ level) [4]. Unfortunately, D decays are limited by phase space to kaon resonances with $M<1.73 \mathrm{GeV}$. B decays to $K n \pi$ final states have ample phase space but are limited by small branching fractions, for example $B_{B^{+} \rightarrow K^{+} \pi^{+} \pi^{-}}=(5.6 \pm 1.0) \cdot 10^{-5}$ 叫. B decays to $J / \psi+K n \pi$ may be more attractive, since they have much larger branching fractions; $B_{B^{+} \rightarrow J / \psi K^{+} \pi^{+} \pi^{-}}=(1.4 \pm 0.6) \cdot 10^{-3}$ and $B_{B^{o} \rightarrow J / \psi K^{+} \pi^{-}}=$ $(1.2 \pm 0.6) \cdot 10^{-3}$ 叫, and the available phase space of $2.18 \mathrm{GeV}$ is adequate for the study of many of the excited kaons discussed here.

There is also interest in the decay systematics of $\mathrm{D}$ and $\mathrm{B}$ mesons to final states such as $\eta K$ and $\eta^{\prime} K$. Lipkin [90, 91] has noted that some heavy-quark weak decay processes involve the strong decay of an intermediate excited kaon, which can lead to unusual branching fractions for these modes. There is already some evidence favoring the resulting selection rules, for example the counterintuitive result $B_{B^{+} \rightarrow \eta^{\prime} K^{+}}=(7.5 \pm 0.7) \cdot 10^{-5}>>B_{B^{+} \rightarrow \eta K^{+}}<6.9 \cdot 10^{-6}, 90 \%$ c.l. (See App.B for a discussion and generalization of Lipkin's results.)

\section{$4.2 \quad 1 S$ States}

The predicted partial width for the transition $K^{*} \rightarrow K \pi$ is somewhat underestimated by the ${ }^{3} \mathrm{P}_{0}$ decay model, as usual for $1^{3} \mathrm{~S}_{1} \rightarrow 1^{1} \mathrm{~S}_{0}+1^{1} \mathrm{~S}_{0}$ decays; this discrepancy was discussed in the section on $1 \mathrm{~S} s \bar{s}$ decays.

\subsection{S States}

\subsection{1 $K^{*}(1414)$, a problematical state}

The $K^{*}(1414)$ is an especially interesting state for future experimental study, since its properties are clearly in disagreement with the expectations of the quark model for a first radial excitation of the $K^{*}(894)$.

This state was first reported at CERN in 1976 as a $1^{-}$enhancement in $\bar{K}^{o} \pi^{+} \pi^{-}$near $1450 \mathrm{MeV}$ [92]. The phase difference between the $1^{-} \pi K^{*}$ and $\rho K$ waves in this mass region was observed to be approximately constant, as required if both arose from a single resonance. However these phases did not show resonant phase motion relative to the clear $2^{+} K_{2}^{*}(1429) \pi K^{*}$ and $\rho K$ amplitudes, which argued against a resonance interpretation of the $1^{-}$enhancement.

A BNL $K^{-} p$ experiment next studied this $1^{-}$enhancement in the final state $K_{S} \pi^{+} \pi^{-}$, again in

the reaction $K^{-} p \rightarrow \bar{K}^{o} \pi^{+} \pi^{-} n$ [93]. Depending on the fit assumptions, the mass and width of the enhancement were found to be $\mathrm{M} \approx 1450-1500 \mathrm{MeV}$ and $\Gamma \approx 170-210 \mathrm{MeV}$. Both $\pi K^{*}$ and $\rho K$ modes were reported, $\pi K^{*}$ being dominant; $B_{\pi K^{*} / \rho K} \sim 5$-7. Possible evidence for resonant phase motion was reported (Fig.12f of Ref. [93]), but the statistics were clearly insufficient for definitive conclusions. 
This was followed by a CERN study of $K^{-} p \rightarrow \bar{K}^{o} \pi^{+} \pi^{-} n$ [94], which confirmed a large $1^{-}$signal in $\pi K^{*}$, and gave a fitted mass and width of $\mathrm{M}=1474 \pm 25 \mathrm{MeV}$ and $\Gamma=257 \pm 65 \mathrm{MeV}$. (See their Table 3; note that this width is reported by the PDG as $275 \mathrm{MeV}$ [1.) This reference concluded that this signal did not seem to be due to a resonance, because the $\rho K$ amplitude did not show the expected resonant phase motion relative to the strong $2^{+} \pi K^{*}$ and $\rho K$ waves. (See Figs. $12 \mathrm{c}$ and 13 of Ref. 94 ).

In 1984 the LASS collaboration also reported a study of $K^{-} p \rightarrow \bar{K}^{o} \pi^{+} \pi^{-} n$ [95]; they found a large $\pi K^{*} 1^{-}$signal with a mass and width of $\mathrm{M}=1412 \pm 9 \pm 2 \mathrm{MeV}$ and $\Gamma=196 \pm 18 \pm 12 \mathrm{MeV}$, and saw no evidence for this state in $\rho K$. The slowly-varying relative $\pi K^{*} 1^{-}$and $2^{+}$phase was attributed to the presence of both $1^{-}$and $2^{+}$resonances, with similar masses and widths. The novel result of this experiment was the lack of a $\rho K K^{*}(1414)$ signal, and it was also noted that the $\pi K$ coupling of the $K^{*}(1414)$ must be very weak. In 1987 LASS reported another study of $K^{-} p \rightarrow \bar{K}^{o} \pi^{+} \pi^{-} n$ [96]; the conclusions regarding the $K^{*}(1414)$ enhancement and the fitted resonance parameters were quite similar to their earlier results in Ref. [95]. A 1988 LASS study of $K^{-} p \rightarrow K^{-} \pi^{+} n$ [97] found that the $K^{*}(1414)$ was weakly coupled to $\pi K$, with a branching fraction of only $(6.6 \pm 1.0 \pm 0.8) \%$. Despite the weak coupling, there was evidence that the $1^{-} \pi^{-} K^{+}$phase motion in this mass region was better described by assuming a $K^{*}$ (1414) resonance (Fig.17 of Ref.[97]). The weak but resonant $\pi K$ coupling of the $K^{*}(1414)$ was also reported by LASS in an unpublished study of $K^{-} p \rightarrow \bar{K}^{o} \pi^{-} n$ [98].

The $K^{*}(1414)$ seems an obvious candidate for the $2{ }^{3} \mathrm{~S}_{1}$ radial excitation of the $K^{*}(894)$, since it is the first strange $1^{-}$vector resonance observed above the $K^{*}$. On closer inspection however there are problems with this identification. First, the $K^{*}(1414)$ mass appears too light if we also accept the $\omega(1419)$ and $\rho(1465)$ as $2{ }^{3} \mathrm{~S}_{1} n \bar{n}$ states; a mass for their strange partner of $c a .1 .55 \mathrm{GeV}$ would appear more plausible. (For example, Godfrey and Isgur 18 found a mass of $1.58 \mathrm{GeV}$ for their $2^{3} \mathrm{~S}_{1}$ kaon.)

A second problem with identifying the $K^{*}(1414)$ with the $2^{3} \mathrm{~S}_{1}$ kaon is that the reported $\pi K$ branching fraction is rather smaller than the ${ }^{3} \mathrm{P}_{0}$-model prediction. In Tables $\mathrm{K} 2$ and $\mathrm{K} 3$ we give predictions for the branching fractions and decay amplitudes of a $2^{3} \mathrm{~S}_{1}$ kaon, assuming masses of 1414 and $1580 \mathrm{MeV}$. The $K^{*}(1414)$ option predicts large and comparable branching fractions to $\pi K, \eta K$, $\rho K$ and $\pi K^{*}$. Although the total width is consistent with that of the $K^{*}(1414)$, the LASS [97] $\pi K$ branching fraction of $(6.6 \pm 1.0 \pm 0.8) \%$ is well below our predicted $28 \%$.

A third problem with identifying the $K^{*}(1414)$ with a $2^{3} \mathrm{~S}_{1}$ kaon, probably the most serious, is the reported strong experimental preference for $\pi K^{*}$ over $\rho K, B_{K^{*}(1414) \rightarrow \rho K / \pi K^{*}}<0.17,95 \%$ c.l. [95]. These are both $2^{3} \mathrm{~S}_{1} \rightarrow{ }^{1} \mathrm{~S}_{0}+{ }^{3} \mathrm{~S}_{1}$ transitions and are within the same $\mathrm{SU}(6)$ multiplets; theoretically these amplitudes are the same function of momenta, and up to phase space corrections these branching fractions should be identical. (Our predicted branching fraction ratio of $B_{K^{*}(1414) \rightarrow \rho K / \pi K^{*}}=0.61$ in Table K2 only departs from unity because of phase space differences.) Although there is a node in this radial transition amplitude, in the ${ }^{3} \mathrm{P}_{0}$ model it is at $\left|\vec{p}_{f}\right|=\sqrt{15 / 2} \beta \approx 1.1 \mathrm{GeV}$, far from the physical final momenta of $\approx 300-400 \mathrm{MeV}$. It is difficult to see how the reported branching fraction ratio can be accommodated given a simple $2^{3} \mathrm{~S}_{1}$ kaon assignment for the $K^{*}(1414)$.

In the tables we also give results for an alternative $K^{*}(1580) 2^{3} \mathrm{~S}_{1}$ state; this higher mass resonance should be rather broad (total width $\approx 350 \mathrm{MeV}$ ), but will again be dominated by decays to $\pi K, \eta K$, $\rho K$ and $\pi K^{*}$, with comparable branching fractions.

This disagreement in mass for the $K^{*}(1414)$ as a $2^{3} \mathrm{~S}_{1}$ kaon is the clearest discrepancy between theory and experiment we find in any of the strange mesons we have considered. If this state is indeed a real $1^{-}$resonance, the low mass may be due to the presence of additional hybrid mixing states, as we noted in the introduction. (The mixing problem for kaons is different from the nonstrange sector 
because of C-parity.) Given the different set of hybrid states available for mixing in the kaon flavor sector, if the mixing is large we would not expect the mass or decay properties to be consistent with the $2^{3} \mathrm{~S}_{1} n \bar{n}$-flavor candidates $\omega(1419)$ and $\rho(1465)$. A comparison of the $\pi K, \pi K^{*}$ and $\rho K$ branching fractions of the $K^{*}(1414)$ with the $\pi \pi$ and $\pi \omega$ branching fractions of the $\rho(1465)$, for example, would be a very interesting test of whether these states appear to belong to the same $\mathrm{SU}(3)$ flavor multiplet. In view of the anomalously low mass of this state, establishing resonant phase motion and accurately determining its decay branching fractions should be a high priority in future experimental studies of the spectrum of strange mesons.

\subsection{2 $K(1460)$}

This state was first reported in 1976 at SLAC by Brandenberg et al. [99] in a PWA of $K^{ \pm} \pi^{+} \pi^{-}$final states produced in $K^{ \pm} p \rightarrow K^{ \pm} \pi^{+} \pi^{-} p$ at $13 \mathrm{GeV}$. The fitted mass and width were $\mathrm{M}=1404 \pm 12 \mathrm{MeV}$ and $\Gamma=232 \pm 16 \mathrm{MeV}$, and the dominant coupling was found to be " $\epsilon K$ ", with some evidence for $\pi K^{*}$ (with a poorly understood $0^{-}$contribution near $1.23 \mathrm{GeV}$ ) and $\rho K$ (which was about $30 \%$ as large in intensity as $\epsilon K$, and peaked at a rather higher mass, about 1.5-1.6 GeV; see their Fig.2c).

This discovery was followed by the ACCMOR analysis of about $200 \mathrm{~K} K^{-} p \rightarrow K^{-} \pi^{+} \pi^{-} p$ events at $63 \mathrm{GeV}$ [75]. The $K^{-} \pi^{+} \pi^{-} 0^{-}$amplitude was fitted assuming the same three modes, $\epsilon K, \pi K^{*}$ and $\rho K$. The estimated mass and width of the $0^{-}$resonance were $M \sim 1.46 \mathrm{GeV}$ and $\Gamma \sim 260$ $\mathrm{MeV}$, with partial widths into each mode of $\Gamma_{\epsilon K}=117 \mathrm{MeV}, \Gamma_{\pi K^{*}}=109 \mathrm{MeV}$ and $\Gamma_{\rho K}=34 \mathrm{MeV}$. Again it was found that that the $\rho K$ signal peaked at a higher mass than $\pi K^{*}$ and $\epsilon K$ (see their Fig.18). Daum et al. noted that the " $\epsilon K$ " mode would also include any contribution from " $\pi \kappa$ ". The Particle Data Group [i] has attributed all of this ACCMOR " $\epsilon K^{\prime}$ " partial width to $\pi K_{0}^{*}(1412)$, although Daum et al. do not make this claim.

The mass of this state is consistent with expectations for a $2^{1} \mathrm{~S}_{0}$ radial excitation of the $K$, assuming that the $\pi(1300)$ is the corresponding pion radial excitation. Similarly, the reported total width of $\sim 250-260 \mathrm{MeV}$ is comparable to the ${ }^{3} \mathrm{P}_{0}$ model expectation of $\Gamma_{\text {tot }} \approx 200 \mathrm{MeV}$ (Table K2).

We have not included the broad $\pi \pi K$ modes " $\epsilon K$ " or " $\pi \kappa$ " in Table K2 because they are closed given our assumed $f_{0}(1370)$ and $K_{0}^{*}(1412)$ masses. (See also Ref. [100] regarding the $\kappa$.) We can test whether these modes are important by assigning a lower mass to the scalars as a width effect. If we assume an $f_{0}(700)$ and a $K_{0}^{*}(1100)$ to model a broad " $\epsilon$ " and " $\kappa$ ", we find very small widths; $\Gamma_{K(1460) \rightarrow \epsilon K}=0.2 \mathrm{MeV}$ and $\Gamma_{K(1460) \rightarrow \pi \kappa}=1.5 \mathrm{MeV}$. There is a node close to the $f_{0}(700) K$ physical point, but the amplitude is nonetheless intrinsically quite small, as is evident from the small $\pi K_{0}^{*}(1100)$ width. Thus the ${ }^{3} \mathrm{P}_{0}$ model is inconsistent with the reports of large $\epsilon K$ or $\pi \kappa$ modes.

The possibility that the " $\epsilon K$ " mode might arise from a nonresonant Deck effect was rejected by Brandenburg et al., as clear resonant phase motion was evident in this channel. A similar situation is found in the decays of the $\pi(1300)$; the ${ }^{3} \mathrm{P}_{0}$ decay model predicts a dominant $\pi \rho$ mode [2]], with $\pi(\pi \pi)_{S}$ only making a small contribution. There actually is a large $0^{-} \pi(\pi \pi)_{S}$ signal in the $1.2 \mathrm{GeV}$ region, which if resonant disagrees with the decay model. The VES collaboration has argued however that the $\pi(\pi \pi)_{S}$ signal may arise from a Deck effect rather than from the $\pi(1300)$ resonance [69].

Daum et al. [75] reported branching fractions of $B_{\pi K^{*}} \sim 42 \%, B_{\rho K} \sim 13 \%$ and $B_{\pi K^{*}} \sim 45 \%$; this preference for $\pi K^{*}$ over $\rho K$ is predicted by the ${ }^{3} \mathrm{P}_{0}$ model, but is expected to be less pronounced. The $\omega K$ mode has not been studied; it would be interesting to study this mode because $\omega K$ is "cleaner" than the modes that have been reported, and the relative strengths of the $\omega K$ and $\rho K$ should be close to the $\mathrm{SU}(3)$ flavor factor of $1 / 3$ of these final states arise dominantly from the $K(1460)$. 


\subsection{S States}

\subsubsection{The unobserved $K^{*}(1950)$}

The masses of the 3S states are not yet well established in any of the light flavor sectors. Here we assume the mass of the experimental $K(1830)$ for our ${ }^{1} \mathrm{~S}_{0}$ state, and with a spin-spin splitting suggested by the $\rho(1465)$ and $\pi(1300) 2 \mathrm{~S}$ candidates we assume a rounded mass of $1950 \mathrm{MeV}$ for the $3^{3} \mathrm{~S}_{1}$ kaon.

This state has many open two-body decay modes, as shown in Table K4. The dominant mode is predicted to be $\rho K^{*}$, with a strong preference for the ${ }^{5} \mathrm{P}_{1}$ final state. The $\omega K^{*}$ mode is also important, suppressed by a flavor factor of $1 / 3$ relative to $\rho K^{*}$. One surprise is that the second mode after $\rho K^{*}$ is predicted to be $\pi K^{*}(1414)$, assuming that this problematical state is indeed the 2S kaon. The very weak $\pi K$ mode is due to a node in the ${ }^{3} \mathrm{P}_{0}$ decay amplitude that is accidentally quite close to the physical point; this also suppresses $\eta K$ and $\eta^{\prime} K$.

The dominance of the $3^{3} \mathrm{~S}_{1} s \bar{s}$ coupling to $\eta^{\prime} K^{*}$ over $\eta K^{*}$ is the consequence of an interesting interference between the $n \bar{n}$ and $s \bar{s}$ components of the $\eta$ and $\eta^{\prime}$, coupled to the spin-one $K^{*}$. This system has a selection rule opposite to that of the more familiar $\eta K$ and $\eta^{\prime} K$ final states, as explained in App.B. With our parameters we predict a branching ratio of $B_{\eta^{\prime} K^{*}} / B_{\eta K^{*}}=24$.

\subsection{2 $K(1830)$}

The ${ }^{3} \mathrm{P}_{0}$ model predicts that this state has a total width of only about $200 \mathrm{MeV}$, and the dominant decay modes are $\rho K^{*}$ and (again rather surprisingly) $\pi K^{*}(1414)$, both with branching fractions of $\approx 20 \%$. A large $\eta K^{*}$ branching fraction is also predicted.

There is an experimental candidate for this state from the CERN Omega Spectrometer [101, reported in their partial wave analysis of the $\phi K$ final state in $K^{-} p \rightarrow K^{+} K^{-} K^{-} p$. A pseudoscalar amplitude with resonant phase motion was observed at a mass and width of $\mathrm{M} \sim 1830 \mathrm{MeV}$ and $\Gamma \sim 250 \mathrm{MeV}$, consistent with our theoretical total width. The predicted branching fraction to this mode is $B_{\phi K}=9 \%$.

\subsection{P States}

\subsection{1 $K_{2}^{*}(1429)$}

Given the success of the ${ }^{3} \mathrm{P}_{0}$ model in describing the strong decays of the $f_{2}(1275)$ [21] and $f_{2}^{\prime}(1525)$, one would expect that the decays of their kaonic partner $K_{2}^{*}(1429)$ would also be well described. This is qualitatively the case; the predicted ordering of partial widths $\pi K>\pi K^{*}>\rho K>\omega K$ is in agreement with experiment, and the predicted and observed values are roughly consistent. (See Table 田)

The detailed agreement with experimental $K_{2}^{*}(1429)$ partial widths however does not appear as impressive as for its $s \bar{s}$ partner $f_{2}^{\prime}(1525)$ (Table 2). This is due to a mismatch between the scales of widths to PsPs and PsV final states that has not been tested in $f_{2}^{\prime}(1525)$ decays, since the partial width for $f_{2}^{\prime}(1525) \rightarrow K K^{*}$ has not been measured.

Note that the partial width to $\eta K$ is very small. This mode is suppressed by destructive interference between the $n \bar{n}$ and $s \bar{s}$ components of the $\eta$, due to the comparable size and opposite sign of the $K_{2}^{*} \rightarrow(n \bar{n}) K$ and $K_{2}^{*} \rightarrow(s \bar{s}) K$ transition amplitudes. The coupling to $\eta^{\prime} K$ in contrast has constructive interference and should be large; unfortunately this mode has no phase space in $K_{2}^{*}(1429)$ decays. Observation of both these modes is possible in decays of higher-mass excited kaons, and the dominant mode depends on the angular quantum numbers of the initial kaon. (See App.B). 


\begin{tabular}{|l|c|c|c|c|c|c|}
\hline mode: $\Gamma_{i}(\mathrm{MeV})$ & $\pi K$ & $\eta K$ & $\rho K$ & $\omega K$ & $\pi K^{*}$ & $\pi \pi K^{*}$ \\
\hline \hline$K_{2}^{*}(1429)($ expt) & $49.1 \pm 1.8$ & $0.15_{-0.10}^{+0.33}$ & $8.5 \pm 0.8$ & $2.9 \pm 0.8$ & $24.3 \pm 1.6$ & $13.2 \pm 2.2$ \\
\hline$K_{2}^{*}(1429)$ (thy) & 56 & 0.57 & 4.4 & 1.2 & 12.8 & - \\
\hline
\end{tabular}

Table 4: Experimental and theoretical partial widths of the $1^{3} \mathrm{P}_{2}$ tensor kaon $K_{2}^{*}(1429)$.

\subsection{2 $\quad K_{0}^{*}(1412)$}

This state is especially interesting due to the controversial status of light scalar mesons in other flavor channels. The $K_{0}^{*}(1412)$ has only been observed in the $\pi K$ mode. The LASS Collaboration [97 found a mass and width of $\mathrm{M}=1412 \pm 6 \mathrm{MeV}$ and $\Gamma=294 \pm 23 \mathrm{MeV}$, and determined a branching fraction of $B_{\pi K}=0.93 \pm 0.04 \pm 0.09$ by assuming that the reaction $K^{-} p \rightarrow \pi^{+} K^{-} n$ was dominated by one pion exchange. This branching fraction is consistent with the ${ }^{3} \mathrm{P}_{0}$ model, which predicts that the other open channel, $\eta K$, has a branching fraction of $\approx 5 \%$.

There is also evidence for the $K_{0}^{*}(1412)$ in $p \bar{p}$ annihilation at rest to $K K \pi$ in several channels, $K_{L} K_{L} \pi^{o}$ [102], $K_{L} K^{ \pm} \pi^{\mp}$ [103], $K^{+} K^{-} \pi^{o}$ [104] and $K_{S} K^{ \pm} \pi^{\mp}$ [105], as summarized in Table 2 of Ref. 104]. Fits to the $K_{0}^{*}$ resonance parameters [102, 103] (specifically T-matrix poles) gave a mass and width of $M \approx 1.42-1.43 \mathrm{GeV}$ and $\Gamma \approx 0.28 \mathrm{GeV}$, very close to the LASS results. It is interesting that the fitted $K K_{0}^{*}(1412)$ contribution to the $p \bar{p} \rightarrow K K \pi$ Dalitz plots is comparable to the $K K^{*}$ contribution; this suggests that $p \bar{p}$ annihilation could be an effective approach for the production of higher-mass excited kaon states, perhaps in future annihilation in flight experiments at GSI 89.].

Our predicted total $K_{0}^{*}(1412)$ width is rather smaller than is observed, $\Gamma_{\text {thy }} \approx 120 \mathrm{MeV}$ versus the LASS result $\Gamma_{\text {expt }}=294 \pm 23 \mathrm{MeV}$ [97]. The amplitude for $1{ }^{3} \mathrm{P}_{0} \rightarrow 1^{1} \mathrm{~S}_{0}+1^{1} \mathrm{~S}_{0}$ however varies rapidly with wavefunction parameter $\beta$ and has a node near $\beta=0.3 \mathrm{GeV}$, so this disagreement is rather sensitive to parameters. We also note that the OGE decay amplitude was found to be especially large in this channel [5]. Since the ${ }^{3} \mathrm{P}_{0}$-model decay amplitude may not be dominant in the decays of light scalar mesons, a comparison to experiment may not be justified in this case.

It is also notable that the $K_{0}^{*}(1412)$ was observed in charmed meson nonleptonic decays by E691 [106] and E687 [107], with a relatively large branching fraction. The PDG [4] reports $B_{D^{+} \rightarrow K_{0}^{*}(1412) \pi^{+}}=$ $(3.7 \pm 0.4) \%$, compared for example to a total $K^{-} \pi^{+} \pi^{+}$branching fraction of $B_{D^{+} \rightarrow K^{-} \pi^{+} \pi^{+}}=$ $(9.1 \pm 0.6) \%$, most of which is nonresonant. In $D^{o} \rightarrow K^{-} \pi^{+} \pi^{o}$ a recent CLEO study found that the largest kaon isobar contribution above the $K^{*}$ was due to the $K_{0}^{*}(1412)$ [100 (see their Table VIII). Of all the excited kaon states above the $K^{*}$ only the low-spin states $K_{0}^{*}(1412), K_{1}(1273), K_{1}(1402)$ and the $K^{*}(1717)$ have been reported in D and B decays; presumably the restriction of the final state to total $\mathrm{J}=0$ suppresses higher- $\mathrm{L}_{q \bar{q}}$ strange states.

\subsection{3 $K_{1}(1273) / K_{1}(1402)$}

The axial kaons $K_{1}(1273)$ and $K_{1}(1402)$ are among the most interesting states in the kaon spectrum. Unlike their $n \bar{n}$ and $s \bar{s}$ flavor partners, the kaons do not have diagonal C-parity, so the spin-singlet $n^{1} P_{1}$ and spin-triplet $n^{3} P_{1}$ basis states mix. This leads to a nontrivial mixing angle $\theta$ for each $n, L$ kaon multiplet, which for $1 \mathrm{P}$ we define by

$$
\left|K_{1}(1273)\right\rangle=+\cos (\theta)\left|1^{1} \mathrm{P}_{1}\right\rangle+\sin (\theta)\left|1^{3} \mathrm{P}_{1}\right\rangle
$$

and

$$
\left|K_{1}(1402)\right\rangle=-\sin (\theta)\left|1^{1} \mathrm{P}_{1}\right\rangle+\cos (\theta)\left|1^{3} \mathrm{P}_{1}\right\rangle
$$




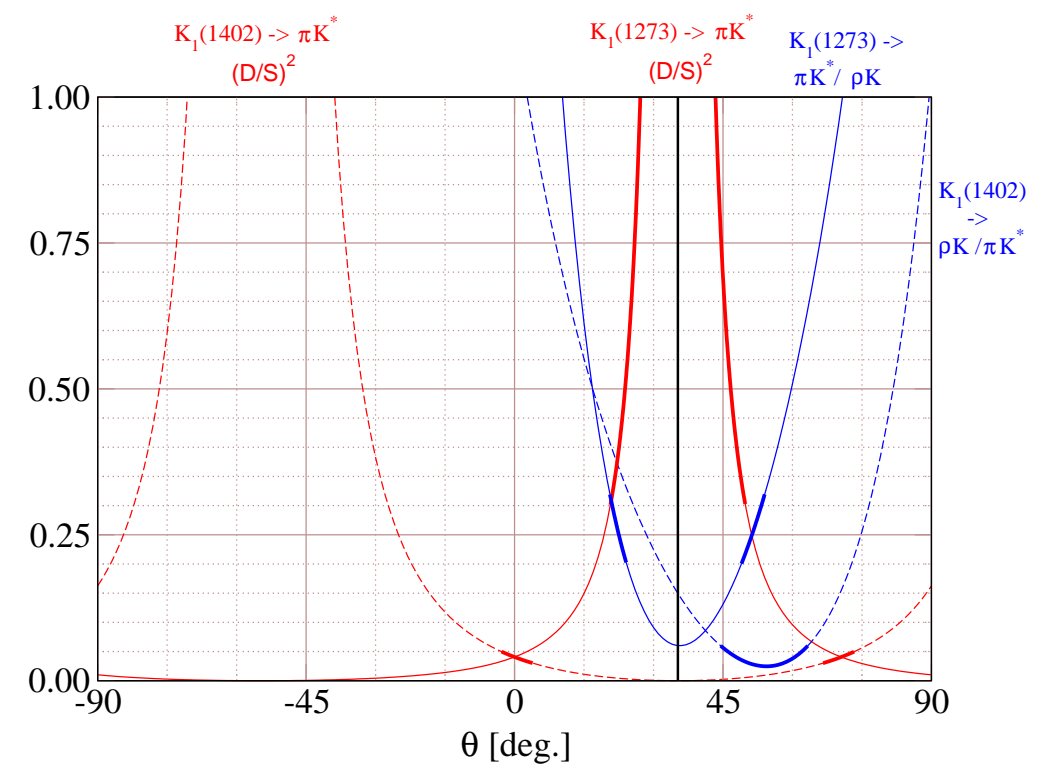

Figure 4. Mixing angle dependence of some $K_{1}$ decay ratios. Regions within $\pm 1 \sigma$ of experiment are indicated by thick lines, and the HQET points $\theta \approx+35.3^{\circ}$ and $\theta \approx-54.7^{\circ}$ are shown as dark verticals.

Although an apparently equivalent mixing angle formula is quoted by Blundell and Godfrey (Eq.(10) of Ref. [17]), our angles are actually opposite in sign because their definition assumes a heavy quark (hence $s \bar{n}=\bar{K}_{1}$, antikaons), whereas we assume this mixing matrix for kaons $\left(n \bar{s}=K_{1}\right)$. This implies opposite signs for $\theta$ in the two conventions because the charge conjugation operator $\mathcal{C}$ gives opposite phases when applied to $\left|{ }^{1} \mathrm{P}_{1}\right\rangle$ and $\left|{ }^{3} \mathrm{P}_{1}\right\rangle$ basis states.

In Tables K6 and K7 we give results for decay amplitudes and widths of the two $K_{1}$ states as functions of $c=\cos (\theta)$ and $s=\sin (\theta)$. Clearly the decay amplitudes and branching fractions depend strongly on this mixing angle. Since the ${ }^{3} \mathrm{P}_{0}$ model is known to give reasonably accurate results for the decay amplitudes of nominally pure ${ }^{3} \mathrm{P}_{1}$ and ${ }^{1} \mathrm{P}_{1}$ states (specifically $a_{1} \rightarrow \rho \pi$ and $b_{1} \rightarrow \omega \pi$, both of which have nontrivial $\mathrm{D} / \mathrm{S}$ amplitude ratios), we can apply the ${ }^{3} \mathrm{P}_{0}$ model to the determination of this mixing angle with some confidence.

There is no theoretical consensus regarding the origin of the mixing angle $\theta$. One speculation, originally due to Lipkin [87, is that it might be determined by the coupling of the two $\left|K_{1}\right\rangle$ states through their decay channels. With sufficiently strong decay couplings the physical resonances can be driven into near "mode eigenstates", which would explain the separation into " $\rho K$ " and " $\pi K^{*}$ " resonances. Under certain simplifying assumptions this picture suggests a singlet-triplet mixing angle of $\theta \approx 45^{\circ}$, essentially the value required by experiment (see Fig.4). Presumably the decay mixing model can be elaborated and applied to the $1 \mathrm{D} K_{2}$ and $2 \mathrm{P} K_{1}$ systems as well, and can be tested when quantitative information becomes available on the strong decays of these states.

Alternatively, it has been noted that the spin-orbit interaction also drives singlet-triplet mixing given unequal quark and antiquark masses, and in the HQET limit $m_{Q} / m_{q} \rightarrow \infty$ one finds "magic mixing angles" of $\theta=\tan ^{-1}(1 / \sqrt{2}) \approx+35.3^{\circ}$ and $\theta=-\tan ^{-1}(\sqrt{2}) \approx-54.7^{\circ}$. The first value is not far from the $\theta$ suggested by $K_{1}$ data (Fig.4). This approximate agreement may be spurious, however, as a mixing angle of $\theta=+5^{\circ}$ is found in the $K_{1}$ system with realistic quark masses [88], which is far from both the HQET value and experiment.

Experimentally, the pattern of decay branching fractions of the $K_{1}(1273)$ and $K_{1}(1402)$ is striking. (See Table 5.) Of the three nominally rather similar PsV modes $\rho K, \omega K$ and $\pi K^{*}$, the $K_{1}(1273)$ 


\begin{tabular}{|l|c|c|c|c|c|}
\hline mode: $\Gamma_{i}(\mathrm{MeV})$ & $\rho K$ & $\omega K$ & $f_{0}(1370) K$ & $\pi K^{*}$ & $\pi K_{0}^{*}(1412)$ \\
\hline \hline$K_{1}(1273)$ (expt) & $38 \pm 10$ & $10 \pm 3$ & $3 \pm 2$ & $14 \pm 6$ & $25 \pm 7$ \\
\hline$K_{1}(1273)$ (thy) & 58 & - & - & 3 & - \\
\hline \hline$K_{1}(1402)$ (expt) & $5 \pm 5$ & $2 \pm 2$ & $3 \pm 3$ & $164 \pm 16$ & not seen \\
\hline$K_{1}(1402)$ (thy) & 30 & 10 & - & 203 & - \\
\hline
\end{tabular}

Table 5: Experimental and theoretical partial widths of the axial kaons $K_{1}(1273)$ and $K_{1}(1402)$. The theoretical numbers assume an HQET mixing angle $\theta=\tan ^{-1}(1 / \sqrt{2}) \approx+35.3^{\circ}$.

shows a strong preference for $\rho K, B_{K_{1}(1273) \rightarrow \pi K^{*} / \rho K}=0.26 \pm 0.06$, whereas the $K_{1}(1402)$ decays almost exclusively to $\pi K^{*}, B_{K_{1}(1402) \rightarrow \rho K / \pi K^{*}}=0.03 \pm 0.03$ [4]. Comparison with the theoretical ${ }^{3} \mathrm{P}_{0}$ model branching fraction ratios in Fig.4 shows that this can be satisfied by a $K_{1}$ singlet-triplet mixing angle of $\theta \approx+45^{\circ}$.

The D/S ratios for the $K_{1}$ states also depend strongly on the singlet-triplet mixing angle. The theoretical ${ }^{3} \mathrm{P}_{0}$-model $|\mathrm{D} / \mathrm{S}|^{2}$ width ratios for $K_{1}(1273) \rightarrow \pi K^{*}$ and $K_{1}(1402) \rightarrow \pi K^{*}$ are shown in Fig.4; these are singular at the two HQET points. The experimental $|\mathrm{D} / \mathrm{S}|^{2}$ ratios of $1.0 \pm 0.7$ for the $K_{1}(1273)$ and $0.04 \pm 0.01$ for the $K_{1}(1402)$ [1] are also indicated, and the data show a strong preference for $\theta=+35^{\circ}$ over $-55^{\circ}$. A more accurate measurement of the $\mathrm{D} / \mathrm{S}$ ratio in $K_{1}(1273) \rightarrow \pi K^{*}$ and a measurement of the sign of $\mathrm{D} / \mathrm{S}$ in $K_{1}(1402) \rightarrow \pi K^{*}$ would be very useful for constraining the singlet-triplet mixing angle.

\subsection{P States}

Recent experimental work, especially from the VES, E852 and Crystal Barrel Collaborations, has established several likely members of the $2 \mathrm{P} n \bar{n}$ multiplet, specifically the $a_{2}(1726)$ 108 (also notable as the first radial excitation reported in $\gamma \gamma$, by L3 at LEP [109]), $a_{1}(1700)$ [69, 70, 110] and $h_{1}(1594)$ [11]. Comparison with their $1 \mathrm{P}$ analogues suggests that the $2 \mathrm{P}-1 \mathrm{P}$ separation is $\approx 450 \mathrm{MeV}$. Presumably the splittings in the kaon system are similar, so we expect the $2 \mathrm{P}$ kaon multiplet at about $1850 \mathrm{MeV}$ for unmixed $2^{3} \mathrm{P}_{\mathrm{J}}$ states and about $1800 \mathrm{MeV}$ for the mean $2{ }^{3} \mathrm{P}_{1}-2^{1} \mathrm{P}_{1}$ mass.

\subsubsection{The unobserved $K_{2}^{*}(1850)$}

We predict that the $2 \mathrm{P}$ tensor state $K_{2}^{*}(1850)$ is rather broad, $\Gamma_{\text {tot }} \approx 370 \mathrm{MeV}$, with no strong preference for any one decay mode. The four largest branching fractions are predicted to be to $\rho K^{*}$, $\pi K^{*}, \rho K$ and $\pi K$, each in the 10-20\% range. Interference between the $|n \bar{n}\rangle$ and $|s \bar{s}\rangle$ components of the $\eta$ and $\eta^{\prime}$ leads to the prediction that $B_{\eta^{\prime} K}>>B_{\eta K}$ (see App.B). Note that there is an inverted rule for the coupling of the $2^{3} \mathrm{P}_{2} s \bar{s}$ to $\eta K^{*}$ relative to $\eta^{\prime} K^{*}$, so we also predict an important $\eta K^{*}$ mode. The theoretically suppressed mode $\eta^{\prime} K^{*}$ is unfortunately not easily accessible in $K_{2}^{*}(1850)$ decays due to the lack of phase space. The predictions that $B_{\eta^{\prime} K}$ and $B_{\eta K^{*}}$ are each $\approx 5 \%$, but that the branching fraction to the lower-mass mode $\eta K$ is much weaker, may serve as useful signatures for this state.

\subsubsection{The unobserved $K_{0}^{*}(1850)$}

The $2^{3} \mathrm{P}_{0}$ scalar $K_{0}^{*}(1850)$ is predicted to have a total width of $\Gamma_{t o t} \approx 450 \mathrm{MeV}$, comparable to the $2^{3} \mathrm{P}_{2}$ tensor. Although the decays of the scalar typically have smaller centrifical barriers, many of 
the tensor decay model are forbidden to the scalar. These compensating effects lead to comparable total widths.

The important decays are again distributed over several modes, but in this case decays to radially and orbitally excited states are expected to dominate. The largest mode is predicted to be $\pi K_{1}(1273)$ (about 30\%), with ca. $10 \%$ branching fractions to $\rho K^{*}, b_{1} K$ and $\pi(1300) K$. The relative strength into $\pi K_{1}(1273)$ versus $\pi K_{1}(1402)$ is strongly dependent on the $1 \mathrm{P}$ mixing angle $\theta$, here assigned the HQET value. The VV modes $\rho K^{*}$ and $\omega K^{*}$ are predicted to have similar ${ }^{1} \mathrm{~S}_{0}$ and ${ }^{5} \mathrm{D}_{0}$ amplitudes. None of the resulting $K+n \pi$ final states is especially attractive experimentally, although $b_{1} K \rightarrow \pi \omega K$ might be interesting as a flux-tube model decay mode expected to show strange hybrids. The $K_{0}^{*}(1850)$ may be observable in its relatively weak $\eta^{\prime} K$ decay, which is also expected to show evidence of the $K_{2}^{*}(1850)$ partner. A much weaker $\eta K$ mode is expected, due to destructive interference in the $\eta$ flavor state (App.B).

The $K_{0}^{*}(1945)$ reported by LASS [97] in $K \pi$ at a mass of $1945 \pm 10 \pm 20 \mathrm{MeV}$ (actually this is an average of two LASS solutions, see their Table 3) is a possible experimental candidate for the $2^{3} \mathrm{P}_{0}$ state. A recent reanalysis of the data found the K-matrix pole at $1885_{-80}^{+50} \mathrm{MeV}$, consistent with the LASS analysis, but the physically more relevant T-matrix pole was found at a mass of $1820 \pm 40$ $\mathrm{MeV}$, with a width of $250 \pm 100 \mathrm{MeV}$ [112]. These parameters are consistent with our expectations for a $2^{3} \mathrm{P}_{0}$ state. The strength of the $\pi K$ coupling reported by LASS is however much larger than our expectations for the $2^{3} \mathrm{P}_{0}$ quark model state; experimentally $B_{K_{0}^{*}(1945) \rightarrow \pi K}=(52 \pm 8 \pm 12) \%$ (again an average of two solutions), whereas the ${ }^{3} \mathrm{P}_{0}$ model predicts a much smaller $B_{\pi K}=6 \%$ for the $2^{3} \mathrm{P}_{0}$ kaon.

\subsection{3 $\quad 2^{3} \mathbf{P}_{1}-2^{1} \mathbf{P}_{1} K_{1}(1800)$ states}

Motivated by the well-known $1^{1} \mathrm{P}_{1}-1^{3} \mathrm{P}_{1}$ mixing in the lighter $1 \mathrm{P} K_{1}$ states, we quote decay amplitudes and partial widths for $2 \mathrm{P} K_{1}(1800)$ states as functions of a similar singlet-triplet mixing angle $\theta$. Our definition of the $2 \mathrm{P}$ mixing angle is

$$
\left|K_{1}^{a}(1800)\right\rangle=+\cos (\theta)\left|2^{1} \mathrm{P}_{1}\right\rangle+\sin (\theta)\left|2^{3} \mathrm{P}_{1}\right\rangle
$$

and

$$
\left|K_{1}^{b}(1800)\right\rangle=-\sin (\theta)\left|2^{1} \mathrm{P}_{1}\right\rangle+\cos (\theta)\left|2^{3} \mathrm{P}_{1}\right\rangle
$$

as was assumed for $1 \mathrm{P}$ states.

It is evident from Table K10 that searches for these resonances might most usefully concentrate on the modes $\rho K$ and $\pi K^{*}$. These branching fractions are intrinsically large, and as their $\cos (\theta) \sin (\theta)$ cross terms have opposite signs, a state that is accidentally suppressed in one mode should be clearly evident in the other. The somewhat weaker $\omega K$ and $\phi K$ modes are rather cleaner to reconstruct, and will be useful as independent checks of the observation of these states. The $\omega K$ partial width is related to $\rho K$ by a trivial isospin factor of $1 / 3$ (with minor phase space differences). The $\phi K$ mode is experimentally attractive because the $\cos (\theta) \sin (\theta)$ cross term in this branching fraction is relatively weak, so we expect both states to be evident in $\phi K$, independent of the mixing angle. This might explain the PDG report of a " $K_{1}(1650)$ " state in $\phi K$ at inconsistent masses of $1650 \pm 50 \mathrm{MeV}$ and $\sim 1840 \mathrm{MeV}$.

The VV modes $\rho K^{*}$ and $\omega K^{*}$ are interesting because the three subamplitudes ${ }^{3} \mathrm{~S}_{1},{ }^{3} \mathrm{D}_{1}$ and ${ }^{5} \mathrm{D}_{1}$ are comparable and are individually proportional to $\cos (\theta)$ or $\sin (\theta)$, and thus may be useful in determining this mixing angle. The mode $\pi K_{1}(1273)$ may also be useful for establishing the $2 \mathrm{P}$ angle, since it couples strongly to the spin-triplet component in the initial state. 


\begin{tabular}{|c|c|c|c|c|c|c|c|c|c|}
\hline mode: $\Gamma_{i}(\mathrm{MeV})$ & $\pi K$ & $\eta K$ & $\eta^{\prime} K$ & $\rho K$ & $\omega K$ & $\pi K^{*}$ & $\rho K^{*}$ & $\omega K^{*}$ & $\pi K_{2}^{*}$ \\
\hline \hline$K_{3}^{*}(1776)(\mathrm{expt})$ & $30 \pm 4$ & $48 \pm 21$ & - & $49 \pm 16$ & - & $32 \pm 9$ & - & - & $<25$ \\
\hline$"$ & & $15 \pm 6[113$ & & & & & & & \\
\hline$K_{3}^{*}(1776)$ (thy) & 40 & 19 & 0.05 & 10 & 3.2 & 14 & 42 & 12 & 1.1 \\
\hline
\end{tabular}

Table 6: Experimental and theoretical partial widths of the $K_{3}^{*}(1776) 1^{3} \mathrm{D}_{3}$ kaon candidate.

Experimental candidates for these $2 \mathrm{P}$ axial-vector states exist, but are rather poorly established. The PDG " $K_{1}(1650)$ " entry summarizes three experimental reports of states at masses of $1650 \pm$ $50 \mathrm{MeV}, \sim 1800 \mathrm{MeV}$ and $\sim 1840 \mathrm{MeV}$, and only the lowest is inconsistent with our assumed $2 \mathrm{P}$ mass. In view of the known experimental splitting of $c a .130 \mathrm{MeV}$ between the two $1 \mathrm{P} K_{1}$ states, these " $K_{1}(1650)$ " reports may well represent observations of the two $\mathrm{J}^{\mathrm{P}}=1^{+} 2 \mathrm{P}$ states.

\section{7 $1 \mathrm{D}$ States}

\subsection{1 $K_{3}^{*}(1776)$}

In view of the reasonably successful ${ }^{3} \mathrm{P}_{0}$-model description of $\phi_{3}(1854)$ decays, one expects a similarly good description of the decays of its kaonic partner $K_{3}^{*}(1776)$. The relatively small total width of the $K_{3}^{*}(1776)$ is indeed reproduced by the model; experimentally it is $\Gamma_{t o t}=159 \pm 21 \mathrm{MeV}$, compared to a theoretical $\Gamma_{\text {tot }}=148 \mathrm{MeV}$.

The PDG reports experimental branching fractions for the $K_{3}^{*}(1776)$, based largely on constrained fits to LASS data. The resulting partial widths are shown in Table 6, together with our predictions. Although discrepancies between theory and experiment appear possible, they are not especially significant at present accuracy. It is notable that the mode with the largest theoretical branching fraction, $\rho K^{*}$, has not been incorporated in the PDG fit. Neglect of this mode will lead to overestimated partial widths for the remaining modes, as the branching fractions are assumed to sum to unity.

In addition to the nine modes given in the summary table, there are several other numerically unimportant ones that are listed in Table K11.

The $\eta K / \eta^{\prime} K$ selection rule (see App.B) is clearly evident theoretically; constructive interference between $n \bar{n}$ and $s \bar{s}$ components of the $\eta$ in this odd-L $\eta K$ state makes $\eta K$ an important mode, whereas $K_{3}^{*} \rightarrow \eta^{\prime} K$ suffers destructive interference and hence is strongly suppressed. (Compare this to the even-L decay mode $K_{2}^{*}(1429) \rightarrow \eta K$ in Table 4 .) The PDG quotes their fitted branching fraction of $B_{K_{3}^{*}(1776) \rightarrow \eta K}=(30 \pm 13) \%$, which combined with their total width gives the $\eta K$ partial width of $48 \pm 21 \mathrm{MeV}$ in our Table 6 . This width is consistent with the $\eta K$ selection rule, albeit with large errors. We note however that there is a better determined $K_{3}^{*}(1776)$ branching fraction ratio published elsewhere by LASS [113], which is $B_{\eta K} / B_{\pi K}=0.50 \pm 0.18$. This gives a partial width of $\Gamma_{K_{3}^{*}(1776) \rightarrow \eta K}=15 \pm 6 \mathrm{MeV}$, which is also quoted in Table 6. Finally, there is an unpublished LASS result of $B_{\eta K} / B_{\pi K}=0.41 \pm 0.053$ [98], which agrees quite well with our theoretical ratio of 0.48 .

\subsection{2 $K^{*}(1717)$}

The PDG considers only $\pi K, \rho K$ and $\pi K^{*}$ modes for this " $K^{*}(1680)$ " state, and previous experimental studies indicate comparable branching fractions to each. Since the $\pi K$ branching fraction was determined by LASS [97] to be $0.388 \pm 0.014 \pm 0.022$, these three modes would appear to account for most of the decays of this state. 


\begin{tabular}{|l|c|c|c|c|c|c|c|c|c|}
\hline mode: $\Gamma_{i}(\mathrm{MeV})$ & $\pi K$ & $\eta K$ & $\eta^{\prime} K$ & $\rho K$ & $\omega K$ & $\phi K$ & $\pi K^{*}$ & $h_{1} K$ & $\pi K_{1}(1273)$ \\
\hline \hline$K^{*}(1717)$ (thy) & 45 & 53 & 1.0 & 26 & 8.5 & 8.6 & 25 & 33 & 145 \\
\hline
\end{tabular}

Table 7: Important theoretical partial widths of a ${ }^{3} \mathrm{D}_{1} K^{*}(1717)$ kaon.

Our decay calculations suggest that this is not correct; we find large couplings to $0^{-} 1^{+}$modes, and the largest branching fraction is predicted to be to $\pi K_{1}(1273)$, with $B_{\pi K_{1}(1273)} \approx 40 \%$. The $\pi K_{1}(1402)$ mode in contrast is predicted to be weak, but this result is strongly dependent on the $K_{1}$ mixing angle $\theta$, here assumed to be equal to the HQET value $\approx 35.3^{\circ}$. If accurately measured, these branching fractions might strongly constrain $\theta$. Unfortunately, the $\pi K_{1}$ modes of the $K^{*}(1717)$ have not been studied experimentally.

This prediction of the ${ }^{3} \mathrm{P}_{0}$ model is familiar in the context of the $1^{3} \mathrm{D}_{1}$ candidate $\rho(1700)$, which is predicted to have very large couplings to $\pi a_{1}$ and $\pi h_{1}$ [21]. Since this large $1^{3} \mathrm{D}_{1} \rightarrow 0^{-} 1^{+}$coupling has not been confirmed experimentally in any flavor sector, the predicted dominance of $\pi K_{1}(1273)$ found here should be considered an interesting future test of the ${ }^{3} \mathrm{P}_{0}$ decay model.

A large $\eta K$ branching fraction and a suppressed $\eta^{\prime} K$ one are predicted, as expected for an oddL final state (App.B). The more important or interesting $K^{*}(1717)$ decay modes (larger than $2 \%$ branching fraction, and the suppressed $\eta^{\prime} K$ mode) are shown in the summary table. For the three reported modes we predict the ordering $\pi K>\rho K \approx \pi K^{*}$, consistent with experiment.

We note in passing that the $K^{*}(1717)$ has been reported in D-meson nonleptonic weak decays, $B_{D^{+} \rightarrow K^{* o}(1717) \pi^{+}}=(1.45 \pm 0.31) \%$ in the $K^{-} \pi^{+}$mode [四, so this approach might allow observation of the interesting modes $\eta K$ (comparable to $\pi K$ ) and $\eta^{\prime} K$ in future (see App.B).

\subsection{3 $\quad K_{2}(1773)$ and $K_{2}(1816)$}

The $K_{2}$ sector is especially interesting because it allows tests of models of mixing between spin-singlet and spin-triplet states, as is seen in the $K_{1}$ system. If this is a short-distance effect we might expect to find much stronger mixing in the $\mathrm{P}$-wave $K_{1}$ system than in the D-wave $K_{2}$ states. The smaller mass splitting in the $K_{2}$ sector suggests that the mixing angle $\theta$ may well be smaller here.

Since the $K_{2}$ states are 400-500 MeV higher in mass, one can measure their couplings to many decay modes that are inaccessible to the $1 \mathrm{P} K_{1}$ states. This will allow many checks of the ${ }^{3} \mathrm{P}_{0}$ decay model, since if it is accurate a single value of the mixing angle $\theta$ should correlate a large number of decays. In Tables K12 and K13 we give results for the decay amplitudes and partial widths of the $K_{2}$ states with general mixing angles,

$$
\left|K_{2}(1773)\right\rangle=+\cos (\theta)\left|1^{1} \mathrm{D}_{2}\right\rangle+\sin (\theta)\left|1^{3} \mathrm{D}_{2}\right\rangle
$$

and

$$
\left|K_{2}(1816)\right\rangle=-\sin (\theta)\left|1^{1} \mathrm{D}_{2}\right\rangle+\cos (\theta)\left|1^{3} \mathrm{D}_{2}\right\rangle .
$$

One can see in the decay tables that many $K_{2}$ partial widths are strongly dependent on the mixing angle $\theta$. The relatively clean modes $\omega K$ and $\phi K$ are especially interesting because their $\sin (\theta) \cos (\theta)$ cross terms have opposite signs, so the ratio $B_{K_{2} \rightarrow \phi K / \omega K}$ depends strongly on the $K_{2}$ mixing angle. The odd-L $\eta K^{*}$ mode is strongly $\theta$-dependent as well, and is predicted to couple dominantly to the spin-singlet ${ }^{1} \mathrm{D}_{2}$ component of the initial $K_{2}$ state (App.B, Table B2). The VV modes $\rho K^{*}$ and $\omega K^{*}$ are interesting because there is no $\sin (\theta) \cos (\theta)$ cross term in the partial widths; the individual subamplitudes are proportional to $\sin (\theta)$ or $\cos (\theta)$ only. A determination of the relative ${ }^{3} \mathrm{P}_{2}$ and ${ }^{5} \mathrm{P}_{2} \mathrm{VV}$ amplitudes would be an excellent independent check of $\theta$, although these modes may be too 
weak to allow this measurement. The $\pi K_{2}^{*}(1429)$ mode is also interesting, because it could be weak or dominant, depending on the value of $\theta$.

Unfortunately the experimental data on the $K_{2}$ states is not yet sufficiently quantitative to be compared usefully to our decay predictions. The PDG claims that $\pi K_{2}^{*}(1429)$ is the dominant $K_{2}(1773)$ decay mode, but the individual experiments are not all in agreement about this. The $\pi K^{*}$, $f_{2}(1275) K, \rho K$ and $\omega K$ modes of the $K_{2}(1773)$ are all "seen", which is at least encouraging for our proposed future determination of $\theta$ from $B_{K_{2}(1773) \rightarrow \phi K / \omega K}$.

The $K_{2}(1816)$ data is even less constraining, with only two experimental references. The modes $\pi K_{2}^{*}(1429), \pi K^{*}, f_{2}(1275) K$ and $\omega K$ are again "seen" in the PDG summary. Daum et al. 75 actually report a strong preference for $\pi K_{2}^{*}(1429), B_{K_{2}(1816) \rightarrow \pi K_{2}^{*} / \pi \pi K} \sim 0.77$. In comparison they quote $B_{K_{2}(1816) \rightarrow f_{2}(1275) K / \pi \pi K} \sim 0.18$ and $B_{K_{2}(1816) \rightarrow \pi K^{*} / \pi \pi K} \sim 0.05$. This large $\pi K_{2}^{*} / \pi K^{*}$ ratio is not consistent with the ${ }^{3} \mathrm{P}_{0}$-model prediction that these two modes have comparable strengths.

The LASS observation [114 of both P- and F-wave contributions to the transition $K_{2}(1773) \rightarrow$ $\omega K$ (Table 2 of Ref.[114]) is quite interesting, as we find that the $\mathrm{F} / \mathrm{P}$ amplitude ratios for both $K_{2} \rightarrow \omega K$ transitions vary rapidly with the singlet-triplet mixing angle $\theta$. Although the LASS results are not very statistically significant (the F-waves are $\approx 1 \sigma$ and $2 \sigma$ from zero), they do show that $\mathrm{F} / \mathrm{P}$ is quite small in $K_{2}(1816) \rightarrow \omega K$. This argues in favor of a sizeable and negative $K_{2}$ mixing angle; a vanishing $K_{2}(1816) \rightarrow \omega K$ F-wave requires $\theta_{2}=-\tan ^{-1}(\sqrt{2 / 3}) \approx-39^{\circ}$.

\section{$4.8 \quad 1 F$ States}

\subsection{1 $K_{4}^{*}(2045)$}

The $K_{4}^{*}(2045)$ is the single well-established member of the $1 \mathrm{~F}$ kaon multiplet. It is assumed to be the flavor partner of the $n \bar{n}$ states $f_{4}(2025)$ and $a_{4}(2011)$ and perhaps the LASS $s \bar{s}$ candidate $f_{4}(2209)$. The reported mass is much closer to the $n \bar{n}$ states than the $s \bar{s}$ candidate, which is surprising, and is reminiscent of the $K^{*}(1410)$.

The PDG total width of $198 \pm 30 \mathrm{MeV}$ is somewhat larger than our theoretical expectation of $\Gamma_{t o t} \approx 100 \mathrm{MeV}$. The ${ }^{3} \mathrm{P}_{0}$ model predicts that only a few low-lying two-body modes of the $K_{4}^{*}(2045)$ have branching fractions larger than a few percent. This weakness of higher-mass modes is typical of a high-L, high-J state, since the angular threshold barriers for decays combined with smaller phase space leads to smaller branching fractions.

The largest modes are predicted to be $\rho K^{*}$ and $\pi K$, with branching fractions of $\approx 30 \%$ and $\approx 20 \%$ respectively. The predicted partial width $\Gamma_{\pi K}=21 \mathrm{MeV}$ is consistent with the reported LASS $\pi K$ branching fraction of $(9.9 \pm 1.2) \%$ [97], given their total width of about $200 \mathrm{MeV}$. We note however that this agreement is rather fortuitous, since this is a G-wave final state and as such has very strong $\left|\vec{p}_{f}\right|^{9}$ threshold behavior. Only three other modes are predicted to be larger than $5 \%$, these being $\omega K^{*}, \rho K$ and $\pi K^{*}$. The $\phi K^{*}$ mode, with a reported branching fraction of $(1.4 \pm 0.7) \%$, can be used to test the assumed flavor independence of the $n \bar{n}$ and $s \bar{s}$ pair production amplitudes in the novel VV channel. Our predicted branching fraction of $2.7 \%$ is consistent with experiment at the current limited accuracy. A comparison of the $\phi K^{*}$ mode with $\rho K^{*}$ or $\omega K^{*}$ would constitute an interesting direct test of the assumed flavor (quark mass) independence of the $q \bar{q}$ pair-production amplitude in the ${ }^{3} \mathrm{P}_{0}$ model, since $\phi K^{*}$ requires $s \bar{s}$ pair production whereas $\rho K^{*}$ and $\omega K^{*}$ require $n \bar{n}$.

It is interesting that the reported PDG branching fractions only account for about half of the $K_{4}^{*}(2045)$ decays. The ${ }^{3} \mathrm{P}_{0}$ model does not anticipate any additional modes with sufficient strength to explain this discrepancy. 


\subsection{2 $\quad K_{2}^{*}(2050)$}

We assume a mass of $2050 \mathrm{MeV}$ for the $1^{3} \mathrm{~F}_{2}$ kaon, which is a rounded $K_{4}^{*}(2045)$ mass. Since this is a high-mass state with low $J$, we find that many two-body final states are predicted to have significant couplings. Axial-vector plus pseudoscalar modes are among the most important; $\pi K_{1}(1273), b_{1} K, a_{1} K$ and $\eta K_{1}(1273)$ (in decreasing order of branching fraction) are all predicted to be in the $\approx 10-30 \%$ range. The S-wave mode $\pi K_{2}(1773)$ is also predicted to have a large $(\approx 20 \%)$ branching fraction, although this is strongly dependent on the $2 \mathrm{P}$ singlet-triplet mixing angle; we have assumed HQET values for the two $K_{2}$ states, analogous to the $K_{1}(1273)$ and $K_{1}(1402)$, and if this is inaccurate there may be a large $\pi K_{2}(1816)$ mode.

The "standard" light modes such as $\pi K$ are predicted to couple rather weakly to this state. $\pi K, \rho K$ and $\pi K^{*}$ have predicted branching fractions of only about $5 \%$. One attractive approach to identifying this state would be to observe it in $\eta^{\prime} K$ (also a $c a .5 \%$ branch), but not in $\eta K$, which is a signature for decays to these even-L final states (App.B).

Should this state be identified, there is an interesting ${ }^{3} \mathrm{P}_{0}$ decay model prediction that the light VV modes $\rho K^{*}, \omega K^{*}$ and $\phi K^{*}$ will couple quite weakly, since they are predicted to have zero coupling in S-wave. We might a priori have expected the S-wave to be the largest VV amplitude.

There is a possible LASS candidate for this state at $1973 \pm 8 \pm 25 \mathrm{MeV}$ [96], reported in $\rho K$ and $\pi K^{*}$, with a total width of $\Gamma_{t o t}=373 \pm 33 \pm 60 \mathrm{MeV}$ and a relative branching fraction of $B_{K_{2}^{*}(1973) \rightarrow \rho K / \pi K^{*}}=1.49 \pm 0.24 \pm 0.09$. These results are consistent with our expectations for a $1^{3} \mathrm{~F}_{2}$ kaon.

\subsubsection{The unobserved $K_{3}(2050)$ states}

The two $\mathrm{J}^{\mathrm{P}}=3^{+} 1 \mathrm{~F}$ states will provide an independent test of models of the mixing between spinsinglet and spin-triplet kaon states, such as is observed in the $1 \mathrm{P} K_{1}(1273)-K_{1}(1402)$ system. We can expect the predicted $1 \mathrm{~F}$ mixing angle to depend rather strongly on the assumed mechanism. If it is a short distance effect it should be much smaller in the $\mathrm{L}=3 K_{3}$ states than in $1 \mathrm{P}$, whereas if it is simply a mixing angle chosen by heavy-quark symmetry, the $1 \mathrm{~F} K_{3}$ and $1 \mathrm{P} K_{1}$ values should be similar. Since the $K_{3}$ states have much higher masses, there are many more decay modes that can be used to determine this mixing angle.

We will assume a mass of $2050 \mathrm{MeV}$ for both $1 \mathrm{~F} K_{3}$ states, so we need only quote results for one linear combination, which we take to be

$$
\left|K_{3}^{a}(2050)\right\rangle=+\cos (\theta)\left|{ }^{1} \mathrm{~F}_{3}\right\rangle+\sin (\theta)\left|{ }^{3} \mathrm{~F}_{3}\right\rangle .
$$

The decay amplitudes and partial widths are given in Table K15 as functions of $\theta$. Note that the total width is not strongly dependent on the mixing angle; we expect these states to have total widths of ca. 200-250 MeV whatever the value of $\theta$.

The PsV modes $\rho K, \omega K, \pi K^{*}$ and $\phi K$ have significant partial widths, and it is notable that the sign of the $\sin (\theta) \cos (\theta)$ cross term is channel-dependent; thus there is especially strong $\theta$-dependence in ratios such as $B_{\phi K} / B_{\omega K}$.

The VV modes have the interesting feature that their partial widths have no $\sin (\theta) \cos (\theta) \operatorname{cross}$ term, because the individual L, S subamplitudes are proportional to either $\sin (\theta)$ or $\cos (\theta)$. (This was also noted for mixed $2 \mathrm{P}$ state decays.) Thus measurements of the $\rho K^{*}$ or $\omega K^{*}$ subamplitudes directly access $\sin (\theta)$ and $\cos (\theta)$.

In the higher-mass final states we find large branching fractions to $a_{2} K, f_{2} K, \pi K_{2}^{*}(1429)$ and $\pi K_{3}^{*}(1776)$, again with strong $\theta$-dependence. The final state $\pi K_{3}^{*}(1776)$ is interesting in that it is the only open $K_{3}(2050)$ mode with an S-wave amplitude. 


\section{$5 \quad$ Summary and Conclusions}

In this paper we have presented a detailed survey of the status and strong decays of all strange mesons expected in the quark model up to $c a .2 .2 \mathrm{GeV}$. This includes the $1 \mathrm{~S}, 2 \mathrm{~S}, 3 \mathrm{~S}, 1 \mathrm{P}, 2 \mathrm{P}, 1 \mathrm{D}$ and $1 \mathrm{~F}$ multiplets of strangeonia and kaonia, making a total of 44 states. 42 of these have strong decays (43 since we consider $\eta_{2}$ flavor mixing), and we have carried out calculations of all the energetically allowed open-flavor decays of all these states in the ${ }^{3} \mathrm{P}_{0}$ model. All independent decay amplitudes and partial and total widths were evaluated numerically and presented in detailed decay tables. In total we have given numerical results for 525 two-body decay modes and 891 decay amplitudes.

This work is intended as a guide for future experimental studies of meson spectroscopy, to indicate what modes and amplitudes are expected to be important and are theoretically interesting, as well as to allow the identification of unusual states such as glueballs and hybrids through their anomalous decay properties.

We have identified several very interesting issues for future experimental studies involving the conventional quark model states. As one example, in the $s \bar{s}$ sector we predict two rather narrow states that have not been identified, the $1^{3} \mathrm{D}_{2} \phi_{2}(1850)$ with $\Gamma_{t o t} \approx 210 \mathrm{MeV}$ (with large $K K^{*}$ and $\eta \phi$ modes) and the $1^{1} \mathrm{D}_{2} \eta_{2}(1850)$ (assuming it is pure $s \bar{s}$; see below) with $\Gamma_{t o t} \approx 130 \mathrm{MeV}$, decaying mainly to $K K^{*}$. The $\eta_{2}$ states at 1617 and $1842 \mathrm{MeV}$ are also very interesting because the highermass state is only seen in $\pi a_{2}$. We consider the effect of a large $n \bar{n} \leftrightarrow s \bar{s}$ mixing angle, and note that this implies important $K K^{*}$ modes that are not evident in the data; the possibility that the highermass $\eta_{2}(1842)$ is a nonstrange hybrid rather than a quarkonium state certainly merits consideration. Future searches for $\mathrm{C}=(-) s \bar{s}$ states might exploit the $\eta \phi$ and $\eta^{\prime} \phi$ " $s \bar{s}$-signature modes", which are not directly accessible to light $n \bar{n}$ mesons.

There are many interesting issues in the kaon sector. One is the amount of spin singlet-triplet mixing in the series of $\mathrm{J}^{\mathrm{P}}=1^{+}, 2^{-}, 3^{+} \ldots$ kaons. The $K_{1}(1273)-K_{1}(1402)$ system is known to have a large singlet-triplet mixing angle, and the physical origin is not well established. Similar mixing is considered in the $2 \mathrm{P}, 1 \mathrm{D}$ " $K_{2}$ " and $1 \mathrm{~F}$ " $K_{3}$ " systems, and it is noted that the decay amplitudes and partial widths of these states are often very sensitive to these mixing angles. Quantitative studes of these strong decay amplitudes and branching fractions will allow the determination of these mixing angles, and can also provide tests of the accuracy of the ${ }^{3} \mathrm{P}_{0}$ decay model.

Kaons are much better established experimentally than $s \bar{s}$ states; of the 21 theoretical excited kaon levels we consider, just eight do not have plausible associated experimental candidates. The eight unknown kaon states are predicted to have total widths in the $\Gamma_{\text {tot }} \approx 300-400 \mathrm{MeV}$ range, and the modes $\rho K, \rho K^{*}$ and $\pi K^{*}$ should be useful for the identification of most of these states. An interesting exception is the $1^{3} \mathrm{~F}_{2} K_{2}^{*}(2050)$, which is predicted to have large branching fractions to the unusual modes $\pi K_{1}(1273), \pi K_{2}(1773)$ and $b_{1} K$.

Kaon decays to modes with an $\eta$ or $\eta^{\prime}$ are especially interesting, in that an interference takes place between the $|n \bar{n}\rangle$ and $|s \bar{s}\rangle$ components of the final $\eta$ or $\eta^{\prime}$. This interference is strongly constructive or destructive depending on the channel and angular quantum numbers, and there is strong experimental evidence of this effect in $K_{2}^{*}(1429)$ decays. The associated selection rules have also been applied to $\mathrm{D}$ and $\mathrm{B}$ meson weak decays to $\eta K$ and related final states, where unusual branching fractions have been observed. We derived these selection rules from our strong decay amplitudes in Appendix B, and noted that there is a nontrivial generalization to modes such as $\eta K_{1}$ in $\mathrm{B}$ decays.

Finally, this work should be useful in searches for glueballs and hybrids, assuming configuration mixing is not large, as one should eliminate the $q \bar{q}$ quark model "background" in any search for new, unconventional meson resonances. We also note that the spectrum of kaons will appear rather different from the spectrum of $n \bar{n}$ or $s \bar{s}$ states if mixing between quarkonia and hybrids $i s$ important, 
because kaonia mix with more hybrid basis states due to the absence of C-parity. This may lead to irregularities in relative level positions in the $n \bar{n}$ and excited kaon spectra, as perhaps is already evident in the low mass of the strange $K^{*}(1414)$ relative to the $n \bar{n}$ state $\rho(1465)$. Irregularities between the kaon and $\mathrm{I}=1 n \bar{n}$ spectra may thus signal the presence of hybrid basis states.

\section{Acknowledgments}

We are pleased to thank W. Bugg, S.U. Chung, F.E. Close, A. Donnachie, W. Dunwoodie, A. Dzierba, S. Godfrey, T. Handler, H. J. Lipkin, R. Mitchell, C.A. Meyer, M. Nozar, W. Roberts, M. Selen, E.S. Swanson, S. Spanier, E. Vaandering and D. Weygand for useful discussions and communications, and C. Salgado for enthusiastic support. This work was supported in part by the United States Department of Energy under contract DE-AC05-96OR22464 managed by UT Battelle at Oak Ridge National Laboratory, by the University of Tennessee, and by the United States Department of Energy under contract W-7405-ENG-36 at Los Alamos National Laboratory.

\section{References}

[1] L. Micu, Nucl. Phys. B10, 521 (1969).

[2] A. LeYaouanc, L. Oliver, O. Pène and J. Raynal, Phys. Rev. D8, 2223 (1973); ibid., D9, 1415 (1974); D11, 680 (1975); D11, 1272 (1975); Phys. Lett. 71B, 397 (1977).

[3] D.V. Amelin et al. (VES), as reported in Ref.[4].

[4] K. Hagiwara et al. (PDG Compilation), Phys. Rev. D66, 010001 (2002).

[5] E.S. Ackleh, T. Barnes and E.S. Swanson, Phys. Rev. D54, 6811 (1996).

[6] M. Nozar et al. (E852), hep-ex/0206026, Phys. Lett. 541B, 35 (2002).

[7] P. Geiger and E.S. Swanson, Phys. Rev. D50, 6855 (1994).

[8] N. Isgur and J. Paton, Phys. Rev. D31, 2910 (1985).

[9] R. Kokoski and N. Isgur, Phys. Rev. D35, 907 (1987).

[10] L. Burakovsky and P.R. Page, Phys. Rev. D62, 014011 (2000); ibid. Eur. Phys. J. C12, 489 (2000).

[11] L. Burakovsky and P.R. Page, Proc. of "rth Conf. on the Intersections of Particle and Nuclear Physics" (CIPANP 2000) (22-28 May, Quebec City, Canada), hep-ph/0007199.

[12] L. Burakovsky and P.R. Page, Phys. Rev. D59, 014022 (1998).

[13] M. Strohmeier-Prešiček, T. Gutsche, A. Faessler and R. Vinh Mau, Phys. Lett. 438B, 21 (1998); Phys. Rev. D60, 054010 (1999).

[14] W. Roberts and B. Silvestre-Brac, Phys. Rev. D57, 1694 (1998); Few Body Sys. 11, 171 (1992).

[15] C. Amsler and F. E. Close, Phys. Rev. D53, 295 (1996); hep-ph/9507326. 
[16] H. Blundell and S. Godfrey, Phys. Rev. D53, 3700 (1996).

[17] H. Blundell, S. Godfrey, and B. Phelps, Phys. Rev. D53, 3712 (1996).

[18] S. Godfrey and N. Isgur, Phys. Rev. D32, 189 (1985).

[19] S. Godfrey, R. Kokoski and N. Isgur, Phys. Lett. 141B, 439 (1984).

[20] G. Busetto and L. Oliver, Z.Phys. C20, 247 (1983).

[21] T. Barnes, F.E. Close, P.R. Page and E.S. Swanson, Phys. Rev. D55, 4157 (1997).

[22] D. Barberis et al. (WA102), Phys. Lett. 471B, 440 (2000).

[23] P.R. Page, E.S. Swanson and A.P. Szczepaniak, Phys. Rev. D59, 034016 (1999).

[24] A.B. Clegg and A. Donnachie, Z. Phys. C62, 455 (1994).

[25] F. Mané et al. (DM1), Phys. Lett. 99B, 261 (1981).

[26] B. Delcourt et al. (DM1), Phys. Lett. 99B, 257 (1981).

[27] P.M. Ivanov et al. (OLYA), Phys. Lett. 107B, 297 (1981).

[28] D. Bisello et al. (DM2), Z. Phys. C39, 13 (1988).

[29] F. Mané et al. (DM1), Phys. Lett. 112B, 178 (1982).

[30] J. Buon et al. (DM1), Phys. Lett. 118B, 221 (1982).

[31] D. Bisello et al. (DM2), Z. Phys. C52, 227 (1991).

[32] A. Antonelli et al. (DM2), Z. Phys. C56, 15 (1992).

[33] D. Aston et al. (Omega Photon), Phys. Lett. 104B, 231 (1981).

[34] M. Atkinson et al. (Omega Photon), Z. Phys. C27, 233 (1985).

[35] J. Busenitz et al. (E401), Phys. Rev. D40, 1 (1989).

[36] J. M. Link et al. (FOCUS), "Observation of a $1750 \mathrm{MeV} / \mathrm{c}^{2}$ Enhancement in the Diffractive Photoproduction of $K^{+} K^{-}$, hep-ex/0208027, Phys. Lett. B (to appear).

[37] M. Frank, N. Isgur, P. J. O’Donnell and J. D. Weinstein, Phys. Rev. D32, 2971 (1985).

[38] G.S.Adams et al. (E852), Phys. Lett. 516B, 264 (2001).

[39] M. Acciarri et al. (L3), Phys. Lett. 501B, 1 (2001), hep-ex/0011035.

[40] E. S. Ackleh and T. Barnes, Phys. Rev. D45, 232 (1992). The theoretical upper limit for $\Gamma_{\gamma \gamma}(\pi(1300))$ in Table I should read $0.49 \mathrm{KeV}$.

[41] C. R. Münz, Nucl. Phys. A609, 364 (1996).

[42] D. M. Coffman et al. (MarkIII), Phys. Rev. D41, 1410 (1990). 
[43] N. Isgur, R. Kokoski and J. Paton, Phys. Rev. Lett. 54, 869 (1985).

[44] T. Barnes, F.E. Close and E. S. Swanson, Phys. Rev. D52, 5242 (1995).

[45] F.E. Close and P.R. Page, Nucl. Phys. B442, 233 (1995).

[46] A.Birman et al. (LASS), Phys. Rev. Lett. 61, 1557 (1988).

[47] F.E. Close and A. Kirk, Z. Phys. C76, 469 (1997).

[48] D. Barberis et al. (WA102), Phys. Lett. 413B, 225 (1997).

[49] D. Barberis et al. (WA102), Phys. Lett. 440B, 225 (1998).

[50] M.Sosa et al. (E690), Phys. Rev. Lett. 83, 913 (1999).

[51] D. Aston et al. (LASS), Phys. Lett. 201B, 573 (1988).

[52] A. Donnachie and Yu. S. Kalashnikova, Proc. of $g^{\text {th }}$ Int. Conf. on Hadron Spectroscopy HADRON 2001, Protvino, Russia, 25 Aug. - 1 Sept. 2001, (AIP, New York, eds. D.Amelin and A.M.Zaitsev), pp.5-14; hep-ph/0110191.

[53] F. E. Close, A. Donnachie and Yu. S. Kalashnikova, Phys. Rev. D65, 092003 (2002), hep$\mathrm{ph} / 0201043$.

[54] R. Bonnaz, B. Silvestre-Brac and C. Gignoux, Eur. Phys. J. A13, 363 (2002), hep-ph/0101112.

[55] The Godfrey-Isgur quark model predicts $\Gamma\left(f_{1}(1426) \rightarrow \gamma \phi\right)=52 \mathrm{keV}$ (S. Godfrey, private communication).

[56] C. J. Morningstar and M. Peardon, Phys. Rev. D60, 034509 (1999); hep-lat/9901004.

[57] C. Amsler, hep-ph/0206104.

[58] W.-J. Lee and D. Weingarten, Phys. Rev. D61, 014015 (2000); hep-lat/9910008. (See especially (8) of hep-lat/9910008; also Weingarten et al. references cited therein.)

[59] D. Barberis et al. (WA102), Phys. Lett. 479B, 59 (2000).

[60] A. Abele et al. (Crystal Barrel), Eur. Phys. J. C19, 667 (2001). The slight discrepancy between the WA102 [59 and Crystal Barrel $f_{0}(1500) \rightarrow \eta \eta^{\prime}$ branching fractions can be attributed to different treatments of phase space in this near-threshold mode (C. A. Meyer, private communication).

[61] J. Sexton, A. Vaccarino and D. Weingarten, Nucl. Phys. Proc. Suppl. 47, 128 (1996); heplat/9602022.

[62] C. Amsler and F. E. Close, Phys. Lett. 353B, 385 (1995); hep-ph/9505219.

[63] M. Acciarri et al. (L3), Phys. Lett. 363B, 118 (1995).

[64] M. Acciarri et al. (L3), Phys. Lett. 501B, 173 (2001). 
[65] H. C. Huang (Belle), $K^{+} K^{-}$and $K_{S} K_{S}$ Production in Two-Photon Collisions at Belle, in Proc. of the 4th Intl. Conf. on B Physics and CP Violation (Ise-Shima, Japan, 19-23 Feb. 2001); hep-ex/0104024.

[66] A. Abele et al. (Crystal Barrel), Phys. Lett. 415B, 280 (1997).

[67] D. Aston et al. (LASS), Nucl. Phys. Proc. Suppl. 21, 5 (1991).

[68] A. Etkin et al. (MPS), Phys. Lett. 201B, 568 (1988).

[69] D.V. Amelin et al. (VES), Phys. Lett. 356B, 595 (1995).

[70] S. U. Chung et al. (E852), Phys. Rev. D65, 072001 (2002).

[71] M. Atkinson et al. (Omega Photon), Nucl. Phys. B231, 15 (1984).

[72] S. Al-Harran et al. (HBC-2M), Phys. Lett. 101B, 357 (1981).

[73] T.A. Armstrong et al. (WA60), Phys. Lett. 110B, 77 (1982).

[74] D.Aston et al. (LASS), Phys. Lett. 208B, 324 (1988).

[75] C. Daum et al. (ACCMOR), Nucl. Phys. B187, 1 (1981).

[76] A. V. Popov (E852), Proc. of $9^{\text {th }}$ Int. Conf. on Hadron Spectroscopy HADRON 2001, Protvino, Russia, 25 Aug. - 1 Sept. 2001, (AIP, New York, eds. D.Amelin and A.M.Zaitsev), pp.565-568.

[77] R.M. Baltrusaitis et al. (MarkIII), Phys. Rev. Lett. 56, 107 (1986).

[78] J.Z. Bai et al. (BES), Phys. Rev. Lett. 76, 3502 (1996).

[79] J.E. Augustin et al. (DM2), Phys. Rev. Lett. 60, 2238 (1988).

[80] C. Evangelista et al. (JETSET), Phys. Rev. D56, 3803 (1997).

[81] C. Evangelista et al. (JETSET), Phys. Rev. D57, 5370 (1998); hep-ex/9802016.

[82] A. Abele et al. (Crystal Barrel), Phys. Lett. 520B, 175 (2001).

[83] P. S. L. Booth et al. (WA67), Nucl. Phys. B273, 677 (1986).

[84] D. Aston et al. (LASS), Phys. Lett. 215B, 199 (1988).

[85] D. Aston et al. (LASS), Nucl. Phys. B301, 525 (1988).

[86] V. N. Nozdrachev (SERP-E173), in Proc. of $9^{\text {th }}$ Int. Conf. on Hadron Spectroscopy HADRON 2001, Protvino, Russia, 25 Aug. - 1 Sept. 2001, (AIP, New York, eds. D.Amelin and A.M.Zaitsev), pp.155-159 (see their Fig.8).

[87] H. J. Lipkin, Phys. Lett. 72B, 250 (1977). See also ibid., Phys. Rev. 176, 1709 (1968).

[88] S. Godfrey and R. Kokoski, Phys. Rev. D43, 1679 (1991). This reference finds that spindependent quark model forces predict a $K_{1}$ mixing angle of $\theta=+5^{\circ}$ (in our conventions), far from the experimentally preferred range in Fig.4. The earlier theoretical value of $+34^{\circ}$ (our conventions) quoted by Godfrey and Isgur in Fig.4 of Ref. 18 is incorrect (S.Godfrey, private communication). 
[89] An International Accelerator Facility for Beams of Ions and Antiprotons, Conceptual Design Report (GSI, November 2001).

[90] H. J. Lipkin, Phys. Rev. Lett. 46, 1307 (1981).

[91] H. J. Lipkin, Phys. Lett. 415B, 186 (1997); Phys. Lett. 433B, 117 (1998); Phys. Lett. 494B, 248 (2000).

[92] J.S.M. Vergeest et al., Phys. Lett. 62B, 471 (1976).

[93] A. Etkin et al. (MPS), Phys. Rev. D22, 42 (1980).

[94] M. Baubillier et al., Nucl. Phys. B202, 21 (1982).

[95] D. Aston et al. (LASS), Phys. Lett. 149B, 258 (1984).

[96] D. Aston et al. (LASS), Nucl. Phys. B292, 693 (1987)

[97] D. Aston et al. (LASS), Nucl. Phys. B296, 493 (1988).

[98] P.F. Bird (LASS), Čerenkov Ring Imaging and Spectroscopy of Charged Kstar Interactions at $11 \mathrm{GeV} / c$. (PhD thesis), SLAC-332 (1989).

[99] G.W. Brandenberg et al. (LASS), Phys. Rev. Lett. 36, 1239 (1976).

[100] S. Kopp et al. (CLEO), Phys. Rev. D63, 092001 (2001). It is also of interest that this reference sees no evidence for a light " $\kappa$ " resonance.

[101] T.A. Armstrong et al. (WA60), Nucl. Phys. B221, 1 (1983).

[102] A. Abele et al. (Crystal Barrel), Phys. Lett. 385B, 425 (1996).

[103] A. Abele et al. (Crystal Barrel), Phys. Rev. D57, 3860 (1998).

[104] A. Abele et al. (Crystal Barrel), Phys. Lett. 468B, 178 (1999).

[105] A. Bertin et al. (OBELIX), Phys. Lett. 434B, 180 (1998).

[106] J.C. Anjos et al. (E691), Phys. Rev. D48, 56 (1993).

[107] P. L. Frabetti et al. (E687), Phys. Lett. 331B, 217 (1994).

[108] C. Amsler et al. (Crystal Barrel), Eur. J. Phys. C23, 29 (2002).

[109] M. Acciarri et al. (L3), Phys. Lett. 413B, 147 (1997).

[110] C.A. Baker et al. (Crystal Barrel), Phys. Lett. 449B, 114 (1999).

[111] P. Eugenio et al. (E852), Phys. Lett. 497B, 190 (2001).

[112] A.V. Anisovich and A.V. Sarantsev, Phys. Lett. 413B, 137 (1997). 
[113] D. Aston et al. (LASS), Phys. Lett. 201B, 169 (1988). The number $\Gamma_{K_{3}^{*} \rightarrow \eta K}=15 \pm 6 \mathrm{MeV}$ we quote follows from combining this reference's branching fraction ratio $B_{\eta K} / B_{\pi K}=0.50 \pm 0.18$ with the PDG total width $\Gamma_{K_{3}^{*}}=159 \pm 21 \mathrm{MeV}$ and $\pi K$ branching fraction $B_{K_{3}^{*} \rightarrow \pi K}=0.188 \pm$ 0.010. The unpublished LASS result $B_{\eta K} / B_{\pi K}=0.41 \pm 0.053$ 98 would lower the experimental partial width to $\Gamma_{K_{3}^{*} \rightarrow \eta K}=12.3 \pm 2.3 \mathrm{MeV}$.

[114] D. Aston et al. (LASS), Phys. Lett. 308B, 186 (1993).

[115] We are indebted to H.J. Lipkin for detailed correspondence regarding this heavy-quark weak decay selection rule. 


\section{Appendix A: ${ }^{3} \mathbf{P}_{0}$ decay model conventions}

In this appendix we discuss some details of the ${ }^{3} \mathrm{P}_{0}$ decay calculations that are presented in this paper. Our diagrammatic, momentum-space formulation of the ${ }^{3} \mathrm{P}_{0}$ model is described in Ref. [5], and our results are essentially an extension of the decay model calculations of Ref. 21], which considered only $n \bar{n}$ mesons in detail.

The ${ }^{3} \mathrm{P}_{0}$ model describes open-flavor meson strong decay as a $q \bar{q}$ pair-production process, in which the new $q \bar{q}$ pair separate into final $q \bar{q}$ mesons $\mathrm{B}$ and $\mathrm{C}$. The pair is assumed to be produced in a $\mathrm{J}^{\mathrm{PC}}=0^{++}$state (hence "3 $\mathrm{P}_{0}$ model"), corresponding to vacuum quantum numbers. This choice is supported by experimental amplitude ratios, notably the D/S ratios in $b_{1} \rightarrow \pi \omega$ [6] and $a_{1} \rightarrow \pi \rho$. As noted in Ref.[5], the usual ${ }^{3} \mathrm{P}_{0}$ decay amplitude is equivalent to the nonrelativistic limit of the interaction Lagrangian $\mathcal{L}_{I}=g \bar{\psi}_{q} \psi_{q}$, with the identification $\gamma=g / 2 m_{q}$. (The dimensionless $\gamma$ is the pair-production amplitude, which is taken to be a free parameter in the ${ }^{3} \mathrm{P}_{0}$ model.) In this

first detailed survey of strange meson decays we have chosen to avoid the complications of moderate parameter variations and the effect of the larger strange quark mass on the meson wavefunctions, and present results that follow from the previously assumed $n \bar{n}$ SHO wavefunctions. Thus the analytical results for amplitudes given in App.A of Ref.[21] are valid for this paper as well. We assume the same SHO wavefunction width parameter and pair-production amplitude as Ref.[21],

$$
\begin{aligned}
& \gamma=0.4 \\
& \beta=0.4 \mathrm{GeV} .
\end{aligned}
$$

Comparison of partial widths to experiment for light decays of strange states shows that the predictions are indeed a useful guide with these parameters. One should of course expect a slight decrease in length scale and hence a slightly larger $\beta$ in the strange mesons, due to the heavier strange quark.

This interaction leads to the two Feynman diagrams of Fig.1 for the process $\mathrm{A} \rightarrow \mathrm{B}+\mathrm{C}$. As shown in Ref. [5], the T-matrix element for each diagram in a given decay is the product of separate factors for flavor, spin, and a convolution integral involving the three mesons' spatial wavefunctions. There is an additional overall "signature" phase of $(-1)$ due to quark operator anticommutation. The color degree of freedom, which would lead to a common overall multiplicative color factor, is suppressed.

The meson flavor states follow the conventions of Ref. 21]. The fundamental quark flavor-3 and antiquark flavor $-\overline{\mathbf{3}}$ are $q=(+d,+u ;+s)$ and $\bar{q}=(+\bar{u},-\bar{d} ;-\bar{s})$, so for example $\left|\pi^{-}\right\rangle=+|d \bar{u}\rangle$ and $\left|K^{-}\right\rangle=+|s \bar{u}\rangle$ but $|\phi\rangle=-|s \bar{s}\rangle,\left|\rho^{+}\right\rangle=-|u \bar{d}\rangle$, and $\left|K^{+}\right\rangle=-|u \bar{s}\rangle$. Unless otherwise stated we take the $\eta$ and $\eta^{\prime}$ to be maximally mixed flavor states,

$$
\begin{aligned}
& |\eta\rangle=\frac{1}{\sqrt{2}}\left(|n \bar{n}\rangle_{0}-|s \bar{s}\rangle\right) \\
& \left|\eta^{\prime}\right\rangle=\frac{1}{\sqrt{2}}\left(|n \bar{n}\rangle_{0}+|s \bar{s}\rangle\right)
\end{aligned}
$$

where the $\mathrm{I}=0$ state $|n \bar{n}\rangle_{0}$ is

$$
|n \bar{n}\rangle_{0}=\frac{1}{\sqrt{2}}(|u \bar{u}\rangle+|d \bar{d}\rangle)
$$

For cases in which we consider the dependence on the $\eta$ - $\eta^{\prime}$ mixing angle we use an expansion in $q \bar{q}$ flavor states, with a flavor mixing angle $\phi$;

$$
|\eta\rangle=\cos (\phi)|n \bar{n}\rangle_{0}-\sin (\phi)|s \bar{s}\rangle
$$


and

$$
\left|\eta^{\prime}\right\rangle=\sin (\phi)|n \bar{n}\rangle_{0}+\cos (\phi)|s \bar{s}\rangle
$$

The more common expansion in $\mathrm{SU}(3)$ octet and singlet flavor states is

$$
|\eta\rangle=\cos \left(\theta_{P}\right)\left|\eta_{8}\right\rangle-\sin \left(\theta_{P}\right)\left|\eta_{1}\right\rangle
$$

and

$$
\left|\eta^{\prime}\right\rangle=\sin \left(\theta_{P}\right)\left|\eta_{8}\right\rangle+\cos \left(\theta_{P}\right)\left|\eta_{1}\right\rangle
$$

Our conventions for these $\mathrm{SU}(3)$ basis states are

$$
\left|\eta_{8}\right\rangle=\sqrt{\frac{1}{6}}(|u \bar{u}\rangle+|d \bar{d}\rangle-2|s \bar{s}\rangle)
$$

and

$$
\left|\eta_{1}\right\rangle=\sqrt{\frac{1}{3}}(|u \bar{u}\rangle+|d \bar{d}\rangle+|s \bar{s}\rangle) .
$$

These expansions imply a relation between $\phi$ and $\theta_{P}$,

$$
\theta_{P}=\phi-\tan ^{-1}(\sqrt{2}) \approx \phi-54.7^{\circ} .
$$

Our maximally mixed states, with $\phi=45^{\circ}$, correspond to the familiar value $\theta \approx-10^{\circ}$.

The strange mesons $K_{1}(1273)$ and $K_{1}(1402)$ also require careful phase definitions, since various conventions have appeared in the literature. We define the singlet-triplet mixing angle $\theta$ for $n \bar{s}$ axial kaon states as

$$
\begin{aligned}
\left|K_{1}(1273)\right\rangle & =+\cos (\theta)\left|1^{1} \mathrm{P}_{1}\right\rangle+\sin (\theta)\left|1^{3} \mathrm{P}_{1}\right\rangle \\
\left|K_{1}(1402)\right\rangle & =-\sin (\theta)\left|1^{1} \mathrm{P}_{1}\right\rangle+\cos (\theta)\left|1^{3} \mathrm{P}_{1}\right\rangle .
\end{aligned}
$$

As we noted in the section on $K_{1}$ mesons, our mixing angle $\theta$ is opposite in sign to that of Blundell and Godfrey [17], because they apply Eqs.(A13,A14) to $s \bar{n}$ antikaons, whereas we apply it to $n \bar{s}$ kaons.

Two physically independent values of $\theta$ follow from the HQET limit $m_{s} \rightarrow \infty$, which are $\theta=$ $+\tan ^{-1}(1 / \sqrt{2}) \approx+35.3^{\circ}$ and $\theta=-\tan ^{-1}(\sqrt{2}) \approx-54.7^{\circ}$. Reference to Fig.4 shows that the data strongly prefer $\theta=\tan ^{-1}(1 / \sqrt{2})$, which gives the HQET $K_{1}$ states

$$
\begin{aligned}
& \left|K_{1}(1273)\right\rangle=+\sqrt{2 / 3}\left|1^{1} \mathrm{P}_{1}\right\rangle+\sqrt{1 / 3}\left|1^{3} \mathrm{P}_{1}\right\rangle \\
& \left|K_{1}(1402)\right\rangle=-\sqrt{1 / 3}\left|1^{1} \mathrm{P}_{1}\right\rangle+\sqrt{2 / 3}\left|1^{3} \mathrm{P}_{1}\right\rangle .
\end{aligned}
$$

This choice assigns the lighter $K_{1}(1273)$ state to the $j_{q}=3 / 2$ multiplet, which may appear surprising since the $j_{q}=3 / 2$ axial is expected to be the higher-mass state in the HQET limit. Of course the HQET limit is difficult to justify for strange quarks; this limit also anticipates a higher-mass ${ }^{3} \mathrm{P}_{2}$ state relative to the $1^{3} \mathrm{P}_{0}$, whereas these are approximately degenerate in the experimental excited kaon spectrum. Our antikaon $K_{1}$ states are taken to be

$$
\begin{aligned}
\left|\bar{K}_{1}(1273)\right\rangle & =-\cos (\theta)\left|1^{1} \mathrm{P}_{1}\right\rangle+\sin (\theta)\left|1^{3} \mathrm{P}_{1}\right\rangle \\
\left|\bar{K}_{1}(1402)\right\rangle & =+\sin (\theta)\left|1^{1} \mathrm{P}_{1}\right\rangle+\cos (\theta)\left|1^{3} \mathrm{P}_{1}\right\rangle
\end{aligned}
$$


with the corresponding HQET states

$$
\begin{aligned}
\left|\bar{K}_{1}(1273)\right\rangle & =-\sqrt{2 / 3}\left|1^{1} \mathrm{P}_{1}\right\rangle+\sqrt{1 / 3}\left|1^{3} \mathrm{P}_{1}\right\rangle \\
\left|\bar{K}_{1}(1402)\right\rangle & =+\sqrt{1 / 3}\left|1^{1} \mathrm{P}_{1}\right\rangle+\sqrt{2 / 3}\left|1^{3} \mathrm{P}_{1}\right\rangle
\end{aligned}
$$

Note the change of the relative sign of the singlet and triplet basis states relative to Eqs.(A15,16). We use these HQET states in calculating decays to $K_{1}$ final states unless otherwise specified. For other excited kaon states with allowed singlet-triplet mixing we treat the mixing angle as a free parameter.

The flavor factors that result from contracting these explicit flavor states using diagrams $\mathrm{d}_{1}$ and $\mathrm{d}_{2}$ of Fig.A1 are given in Table A1 for the strange decays of interest here.

\begin{tabular}{||l|l|c|c|c||}
\hline \multicolumn{5}{||c|}{ Table A1. Flavor weight factors for strange meson decays. } \\
\hline Generic Decay & Example & $I_{\text {flavor }}\left(d_{1}\right)$ & $I_{\text {flavor }}\left(d_{2}\right)$ & $\mathcal{F}$ \\
\hline \hline$(s \bar{s}) \rightarrow(n \bar{s})(s \bar{n})$ & $\phi \rightarrow K^{+} K^{-}$ & +1 & 0 & 2 \\
\hline$(s \bar{s}) \rightarrow(n \bar{s})(s \bar{n})^{\prime}$ & $\phi(1680) \rightarrow K^{+} K^{*-}$ & +1 & 0 & 4 \\
\hline$(s \bar{s}) \rightarrow \eta \eta$ & $f_{2}^{\prime}(1525) \rightarrow \eta \eta$ & $-1 / 2$ & $-1 / 2$ & $1 / 2$ \\
\hline$(s \bar{s}) \rightarrow \eta \eta^{\prime}$ & $f_{2}^{\prime}(1525) \rightarrow \eta \eta^{\prime}$ & $+1 / 2$ & $+1 / 2$ & 1 \\
\hline$(s \bar{s}) \rightarrow \eta^{\prime} \eta^{\prime}$ & $f_{4}(2200) \rightarrow \eta^{\prime} \eta^{\prime}$ & $-1 / 2$ & $-1 / 2$ & $1 / 2$ \\
\hline$(s \bar{s}) \rightarrow \eta(s \bar{s})$ & $\phi(1680) \rightarrow \eta \phi$ & $-1 / \sqrt{2}$ & $-1 / \sqrt{2}$ & 1 \\
\hline$(s \bar{s}) \rightarrow \eta^{\prime}(s \bar{s})$ & $\phi(2050) \rightarrow \eta^{\prime} \phi$ & $+1 / \sqrt{2}$ & $+1 / \sqrt{2}$ & 1 \\
\hline$(s \bar{s}) \rightarrow(s \bar{s})(s \bar{s})$ & $f_{4}(2200) \rightarrow \phi \phi$ & -1 & -1 & $1 / 2$ \\
\hline$(s \bar{s}) \rightarrow(s \bar{s})(s \bar{s})^{\prime}$ & - & -1 & -1 & 1 \\
\hline$(n \bar{s}) \rightarrow(n \bar{n})_{I=1}(n \bar{s})$ & $K^{*+} \rightarrow \pi^{o} K^{+}$ & 0 & $+1 / \sqrt{2}$ & 3 \\
\hline$(n \bar{s}) \rightarrow(n \bar{n})_{I=0}(n \bar{s})$ & $K_{3}^{*+} \rightarrow \omega K^{+}$ & 0 & $+1 / \sqrt{2}$ & 1 \\
\hline$(n \bar{s}) \rightarrow \eta(n \bar{s})$ & $K_{3}^{*+} \rightarrow \eta K^{+}$ & $-1 / \sqrt{2}$ & $+1 / 2$ & 1 \\
\hline$(n \bar{s}) \rightarrow \eta^{\prime}(n \bar{s})$ & $K_{3}^{*+} \rightarrow \eta^{\prime} K^{+}$ & $+1 / \sqrt{2}$ & $+1 / 2$ & 1 \\
\hline$(n \bar{s}) \rightarrow(s \bar{s})(n \bar{s})$ & $K_{3}^{*+} \rightarrow \phi K^{+}$ & -1 & 0 & 1 \\
\hline
\end{tabular}

The amplitudes quoted in the detailed decay tables are just the $\left\{\mathcal{M}_{L S}\right\}$ amplitudes of Ref. [5], in units of $\left[\mathrm{GeV}^{-1 / 2}\right]$. Amplitude ratios allow sensitive tests of the nature of a resonance, so it is important to determine these with well-defined relative phases. To quote specific amplitudes in the decay tables we have specialized to particular charge states, which are illustrated by the examples in Table A1. Note that the BC ordering is important; if we exchange mesons B and C in Table A1 or in the decay tables, we change the phases of the decay amplitudes. To obtain a unique set of phases we define $\hat{\Omega}$ as the recoil direction of meson $\mathrm{B}$ (with $\mathrm{C}$ along $-\hat{\Omega}$ ), and the amplitudes are taken to be the coefficients of the T-matrix expansion in orthonormal angular momentum eigenfunctions $\left\{f_{\mathrm{JLS}}(\hat{\Omega})\right\}$. For spinless final mesons $\mathrm{B}$ and $\mathrm{C}$, these amplitudes are the coefficients of a spherical harmonic expansion.

The total decay rate is given by the T-matrix amplitude squared, integrated over final angles, summed over all final spin and charge states, and multiplied by the physical, relativistic phase space; again this procedure is described in detail in Ref. [5]. (We note in passing that the $\rho^{+} \rightarrow \pi^{+} \pi^{o}$ example in that reference has a typographical error in Eq.(A17); the factor of $M_{\rho}$ should be $E_{B} E_{C} / M_{A}=$ $M_{\rho} / 4$, as stated in the subsequent text.) Since we neglect mass splittings within an isomultiplet, the sum over charge states acts as a simple multiplier of the partial width into the specific charge channel used as our example; this multiplier is quoted as $\mathcal{F}$ in Table A1. This $\mathcal{F}$ also incorporates the $1 / 2$ ! statistical factor present if $\mathrm{B}$ and $\mathrm{C}$ are identical. The actual light meson masses used here 

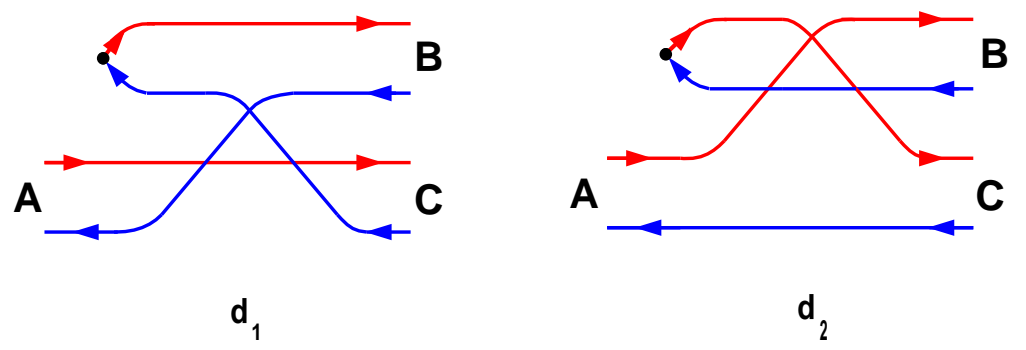

Figure A1. The two meson decay diagrams in the ${ }^{3} \mathrm{P}_{0}$ model.

are $m_{\pi}=138 \mathrm{MeV}, m_{K}=496 \mathrm{MeV}, m_{\eta}=547 \mathrm{MeV}, m_{\rho}=770 \mathrm{MeV}, m_{\omega}=782 \mathrm{MeV}, m_{K^{*}}=$ $894 \mathrm{MeV}, m_{\eta^{\prime}}=958 \mathrm{MeV}, m_{\phi}=1019 \mathrm{MeV}, m_{f_{2}}=1275 \mathrm{MeV}, m_{f_{1}}=1282 \mathrm{MeV}, m_{f_{0}}=1370 \mathrm{MeV}$, $m_{h_{1}}=1170 \mathrm{MeV}, m_{a_{2}}=1318 \mathrm{MeV}, m_{a_{1}}=1230 \mathrm{MeV}, m_{a_{0}}=1450 \mathrm{MeV}$ and $m_{b_{1}}=1230 \mathrm{MeV}$. For the less familiar higher-mass states we used the resonance label to display the assumed mass. For example, the $K^{*}(1414)$ entries in the decay tables imply that we assumed a $K^{*}$ mass of $1414 \mathrm{MeV}$ in our decay calculations. 


\section{Appendix B: Selection rules for decays to $\eta K^{(*)}$ and $\eta^{\prime} K^{(*)}$}

\section{B1. Introduction}

The relative branching fractions of decays of an excited kaon to the pairs of modes $\left(\eta K, \eta^{\prime} K\right)$ and $\left(\eta K^{*}, \eta^{\prime} K^{*}\right)$ are very interesting, in that they involve constructive or destructive interference between the $|n \bar{n}\rangle$ and $|s \bar{s}\rangle$ internal components of the $\eta$ or $\eta^{\prime}$ meson. Lipkin [90, 91] has previously discussed this effect in the context of heavy-quark ( $\mathrm{D}$ and $\mathrm{B}$ ) nonleptonic weak decays. In this appendix we consider the application to excited kaon strong decays, and derive the associated selection rules. The results are counterintuitive, in that the higher-mass $\eta^{\prime}$ decay mode is often favored over the $\eta$ mode.

\section{B2. $\eta K$ and $\eta^{\prime} K$ final states}

To illustrate these selection rules, we first consider the decay of a generic excited kaon $K^{*+}$, with flavor state $-|u \bar{s}\rangle$, to $\eta K^{+}$. (The $K^{*+}$ must have $S_{u \bar{s}}=1$ because the spin matrix element $S_{\mathrm{A}}=$ $0 \rightarrow\left(S_{\mathrm{B}}, S_{\mathrm{C}}\right)=(0,0)$ vanishes in the ${ }^{3} \mathrm{P}_{0}$ model. $)$ We first attach the flavor state vectors

$$
\begin{aligned}
& |\mathrm{A}\rangle=\left|K^{*+}\right\rangle=-|u \bar{s}\rangle \\
& |\mathrm{B}\rangle=|\eta\rangle=\cos (\phi) \frac{1}{\sqrt{2}}(|u \bar{u}\rangle+|d \bar{d}\rangle)-\sin (\phi)|s \bar{s}\rangle \\
& |\mathrm{C}\rangle=\left|K^{+}\right\rangle=-|u \bar{s}\rangle
\end{aligned}
$$

to the two Feynman diagrams in Fig.A1. Evidently diagram $d_{1}$ only couples to the $|s \bar{s}\rangle$ component of the $\eta$, requires $s \bar{s}$ pair production, and gives a flavor factor (flavor matrix element) of $-\sin (\phi)$. Following Lipkin [90], we also assume an $s \bar{s}$ pair-production suppression factor of $\xi$. Diagram $d_{2}$ instead requires $u \bar{u}$ pair production and only couples to the $|u \bar{u}\rangle$ component of the $\eta$, giving a flavor factor of $+\cos (\phi) / \sqrt{2}$. Combining these factors, and carrying out this exercise for the $\eta^{\prime}$ as well, we find the generalized flavor factors given in Table B1.

\begin{tabular}{||l|c|c||}
\hline \multicolumn{3}{||c|}{ Table B1. Generalized $\eta$ and $\eta^{\prime}$ flavor factors. } \\
\hline Channel & $I_{\text {flavor }}\left(d_{1}\right)$ & $I_{\text {flavor }}\left(d_{2}\right)$ \\
\hline \hline$K^{\prime *+} \rightarrow \eta K^{+}$ & $-\sin (\phi) \xi$ & $+\cos (\phi) / \sqrt{2}$ \\
\hline$K^{\prime *-} \rightarrow \eta^{\prime} K^{-}$ & $+\cos (\phi) \xi$ & $+\sin (\phi) / \sqrt{2}$ \\
\hline
\end{tabular}

Since $\sin (\phi) \approx \cos (\phi)$ in practice, it is clear from the table that decays to $\eta K$ would experience destructive interference between diagrams $d_{1}$ and $d_{2}$ if the flavor factors were the only relevant variables. Conversely, decays to $\eta^{\prime} K$ experience constructive flavor interference. If the amplitudes associated with diagrams $d_{1}$ and $d_{2}$ were equal, neglecting phase space differences the branching fraction ratio would be

$$
\frac{B_{\eta K}}{B_{\eta^{\prime} K}}=\left(\frac{1-\sqrt{2} \xi \tan (\phi)}{\tan (\phi)+\sqrt{2} \xi}\right)^{2} .
$$

For maximally mixed states $(\tan (\phi)=1)$ without $s \bar{s}$ pair-production suppression $(\xi=1)$ this ratio is

$$
\frac{B_{\eta K}}{B_{\eta^{\prime} K}}=\left(\frac{1-\sqrt{2}}{1+\sqrt{2}}\right)^{2} \approx 0.029,
$$

which shows that this interference can have a dramatic effect.

Of course the amplitudes associated with diagrams $d_{1}$ and $d_{2}$ are not equal in general. They instead have diagram-dependent, coupled spin factors and spatial overlap integrals, which in the ${ }^{3} \mathrm{P}_{0}$ model are CG-weighted sums of terms of the form 


$$
I_{\text {spin }+ \text { space }}\left(d_{1}\right)=\left.\langle s \bar{s}|\vec{\sigma}| 0\rangle\right|_{d_{1}} \cdot \int d^{3} k \phi_{A}(2 \vec{k}-2 \vec{B}) \phi_{B}^{*}(2 \vec{k}-\vec{B}) \phi_{C}^{*}(2 \vec{k}-\vec{B}) \vec{k}
$$

and

$$
I_{\text {spin+space }}\left(d_{2}\right)=\left.\langle s \bar{s}|\vec{\sigma}| 0\rangle\right|_{d_{2}} \cdot \int d^{3} k \phi_{A}(2 \vec{k}+2 \vec{B}) \phi_{B}^{*}(2 \vec{k}+\vec{B}) \phi_{C}^{*}(2 \vec{k}+\vec{B}) \vec{k} .
$$

Here $\langle s \bar{s}|\vec{\sigma}| 0\rangle$ is the spin-vector matrix element of the $q \bar{q}$ pair produced in the spin state implied by each diagram. We can take the $K^{*+} \rightarrow \eta K^{+}$and $K^{*+} \rightarrow \eta^{\prime} K^{+}$overlaps $I_{\text {spin+space }}$ to be single terms (B6) and (B7) for each diagram without loss of generality because the final mesons factor as $\phi_{L L_{z}=00}(\vec{p}) \chi_{S S_{z}=00}$, and the initial meson $K^{*+}$ is the sum of $\phi_{L L z}(\vec{p}) \chi_{1 S_{z}}$ factored components that can be treated individually as the initial $K^{*+}$. Each $K^{*+}$ component gives a single $\langle s \bar{s}|\vec{\sigma}| 0\rangle=$ $\left\langle\chi_{00} \chi_{00}|\vec{\sigma}| \chi_{1 S_{z}}\right\rangle$ matrix element, which is the same for both diagrams because the final $\eta K$ or $\eta^{\prime} K$ spin state $\left|\chi_{00} \chi_{00}\right\rangle$ is symmetrical.

The $d_{1}$ and $d_{2}$ spatial overlap integrals evidently satisfy

$$
\vec{I}_{\text {space }}^{\left(d_{1}\right)}(\vec{B})=\vec{I}_{\text {space }}^{\left(d_{2}\right)}(-\vec{B})
$$

for any set of meson spatial wavefunctions. Since these integrals are related by parity, and the final states we are considering have definite parity $(-1)^{\mathrm{L}_{B C}}$, we may remove a common factor and find for the $K^{*+} \rightarrow \eta K^{+}$decay amplitude

$$
\mathcal{A}_{K^{\prime *+} \rightarrow \eta K^{+}} \propto\langle s \bar{s}|\vec{\sigma}| 0\rangle \cdot \vec{I}_{\text {space }}^{\left(d_{1}\right)}(\vec{B})\left\{-\xi \sin (\phi)+(-1)^{\mathrm{L}_{B C}} \frac{1}{\sqrt{2}} \cos (\phi)\right\}
$$

and similarly for $K^{*+} \rightarrow \eta^{\prime} K^{+}$

$$
\mathcal{A}_{K^{\prime *+} \rightarrow \eta^{\prime} K^{+}} \propto\langle s \bar{s}|\vec{\sigma}| 0\rangle \cdot \vec{I}_{\text {space }}^{\left(d_{1}\right)}(\vec{B})\left\{+\xi \cos (\phi)+(-1)^{\mathrm{L}_{B C}} \frac{1}{\sqrt{2}} \sin (\phi)\right\} .
$$

Neglecting phase space differences, the $\eta K / \eta^{\prime} K$ branching fraction ratio is again the amplitude ratio squared,

$$
\frac{B_{\eta K}}{B_{\eta^{\prime} K}}=\left(\frac{1-(-1)^{\mathrm{L}_{B C}} \sqrt{2} \xi \tan (\phi)}{\tan (\phi)+(-1)^{\mathrm{L}_{B C}} \sqrt{2} \xi}\right)^{2},
$$

which generalizes the S-wave result (B4). This agrees with Lipkin's Eq.(10b) in Ref. 90], in which the maximally-mixed case $\tan (\phi)=1$ was assumed. Evidently the general rule is that odd-L final states favor $\eta K$, and even-L favors $\eta^{\prime} K$.

Observation of the enhanced $\eta^{\prime} K$ modes requires the study of even-J kaonia with masses well above $M_{\eta^{\prime}}+M_{K}=1.45 \mathrm{GeV}$. Only four of the states we have considered in this paper satisfy these requirements, the $2^{3} \mathrm{P}_{2}, 2^{3} \mathrm{P}_{0}, 1^{3} \mathrm{~F}_{4}$ and $1^{3} \mathrm{~F}_{2}$. Of these only the $1^{3} \mathrm{~F}_{4}$ has a widely accepted experimental candidate, the $K_{4}^{*}(2045)$. Unfortunately the $K_{4}^{*}(2045) \rightarrow \eta^{\prime} K$ branching fraction is predicted to be quite small, due to the G-wave centrifical barrier. Identification of these as yet unknown $2 \mathrm{P}$ and $1^{3} \mathrm{~F}_{2}$ states could prove difficult because they are all expected to be rather broad, with $\Gamma_{t o t} \approx 300-400 \mathrm{MeV}$. In part because of these large widths the theoretical branching fractions of these states to the enhanced $\eta^{\prime} K$ modes are unfortunately not especially large; all are $3-5 \%$.

It will be easier to test the selection rule on $\eta K$ states alone, since this mode has a much lower threshold of $\approx 1.04 \mathrm{GeV}$. The branching fraction ratio relative to $\pi K$, which provides a convenient reference, is

$$
\frac{B_{\eta K}}{B_{\pi K}}=\frac{1}{3}\left(\cos (\phi)-(-1)^{\mathrm{L}_{B C}} \sqrt{2} \xi \sin (\phi)\right)^{2},
$$


if we again neglect phase space differences. For the maximally-mixed case with no $s \bar{s}$ suppression this becomes

$$
\frac{B_{\eta K}}{B_{\pi K}}=\frac{1}{6}\left(3-(-1)^{\mathrm{L}_{B C}} 2 \sqrt{2}\right)
$$

which shows that $\eta K$ will be comparable to $\pi K$ in strength in odd- $\mathrm{L}_{\eta K}$ modes (decays of odd-J kaons), but a factor of $\approx 35$ smaller than $\pi K$ in decays of even-J kaons. Of course this simple estimate should be corrected for phase space, which usually leads to additional suppression of the $\eta K$ mode relative to $\pi K$.

A very weak $\eta K$ mode has already been reported in the decay of the even-J $K_{2}^{*}(1429)$, as expected (Table 4). This suppression of $\eta K$ in even-L final states could also be tested in $K_{4}^{*}(2045)$ decays. In contrast we should see large $\eta K$ branching fractions in odd-L final states, arising for example from decays of the odd-J spin-triplet states $K^{*}(1414)$ (assuming this actually is the $2^{3} \mathrm{~S}_{1}$ kaon), the $K_{3}^{*}(1776)$ and the $K^{*}(1717)$. The $K_{3}^{*}$ is especially attractive for this study because it is relatively narrow, and there is already evidence from LASS 113 that the $K_{3}^{*} \rightarrow \eta K$ mode is enhanced approximately as expected; the PDG width and $\pi K$ branching fraction, combined with the LASS $B_{\eta K} / B_{\pi K}$ ratio 113 correspond to $\Gamma_{K_{3}^{*} \rightarrow \eta K}=15 \pm 6 \mathrm{MeV}$, consistent with our theoretical prediction of $19 \mathrm{MeV}$ (see Table 6).

\section{B3. $\eta K^{*}$ and $\eta^{\prime} K^{*}$ final states}

\section{B3.1 Derivation}

The selection rules for decays to $\eta$ and $\eta^{\prime}$ and an $S_{q \bar{q}}=1$ " $K^{*}$ " kaon are rather more complicated, as they depend on the $S_{q \bar{q}}$ of the initial kaon as well. The difference from the previous case is due to the modified spin matrix elements. The decays $K^{*} \rightarrow \eta K$ and $\eta^{\prime} K$ involved the spin matrix elements $\langle s \bar{s}|\vec{\sigma}| 0\rangle=\left\langle\chi_{00} \chi_{00}|\vec{\sigma}| \chi_{1 S_{z}}\right\rangle$, which were identical for diagrams $d_{1}$ and $d_{2}$,

$$
\left.I_{s p i n}^{\left(d_{1}\right)}\right|_{K^{*} \rightarrow \eta^{(\prime)} K}=\left.I_{s p i n}^{\left(d_{1}\right)}\right|_{K^{*} \rightarrow \eta^{(\prime)} K}
$$

For a transition of the type $K \rightarrow \eta^{(\prime)} K^{*}$ we have a spin matrix element $\langle s \bar{s}|\vec{\sigma}| 0\rangle=\left\langle\chi_{00} \chi_{1 S_{z}}|\vec{\sigma}| \chi_{00}\right\rangle$, which is again identical for each diagram,

$$
\left.I_{\text {spin }}^{\left(d_{1}\right)}\right|_{K \rightarrow \eta^{\left({ }^{\prime}\right)} K^{*}}=\left.I_{\text {spin }}^{\left(d_{2}\right)}\right|_{K \rightarrow \eta^{\left({ }^{\prime}\right)} K^{*}} .
$$

Since the spatial matrix elements are identical, in this case we have a result analogous to the previous $\eta K$ and $\eta^{\prime} K$ result (again neglecting phase space differences for illustration),

$$
\frac{B_{K \rightarrow \eta K^{*}}}{B_{K \rightarrow \eta^{\prime} K^{*}}}=\left(\frac{1-(-1)^{\mathrm{L}_{B C}} \sqrt{2} \xi \tan (\phi)}{\tan (\phi)+(-1)^{\mathrm{L}_{B C}} \sqrt{2} \xi}\right)^{2},
$$

so that decays of a spin-singlet " $K$ " favor $\eta K^{*}$ in odd- $\mathrm{L}_{B C}$ channels and $\eta^{\prime} K^{*}$ in even- $\mathrm{L}_{B C}$.

For transitions from spin-triplet " $K^{*}$ " states to $\eta K^{*}$ and $\eta^{\prime} K^{*}$ the rule is inverted; in this case the spin matrix elements are $\langle s \bar{s}|\vec{\sigma}| 0\rangle=\left\langle\chi_{00} \chi_{1 S_{z}^{\prime}}|\vec{\sigma}| \chi_{1 S_{s}}\right\rangle$, which are opposite in sign between diagrams,

$$
\left.I_{\text {spin }}^{\left(d_{1}\right)}\right|_{K^{*} \rightarrow \eta^{\left({ }^{\prime}\right)} K^{*}}=-\left.I_{\text {spin }}^{\left(d_{2}\right)}\right|_{K^{*} \rightarrow \eta^{\left({ }^{\prime}\right)} K^{*}} .
$$


This change of relative sign between diagrams generalizes the reduced branching fraction ratio to

$$
\frac{B_{K^{*} \rightarrow \eta K^{*}}}{B_{K^{*} \rightarrow \eta^{\prime} K^{*}}}=\left(\frac{1+(-1)^{\mathrm{L}_{B C}} \sqrt{2} \xi \tan (\phi)}{\tan (\phi)-(-1)^{\mathrm{L}_{B C}} \sqrt{2} \xi}\right)^{2},
$$

so decays of a spin-triplet " $K^{*}$ " instead favor $\eta K^{*}$ in even- $\mathrm{L}_{B C}$ and $\eta^{\prime} K^{*}$ in odd- $\mathrm{L}_{B C}$.

Our results for these $\eta$ and $\eta^{\prime}$ modes in all cases are summarized in Table B2. Again $K$ and $K^{*}$ refer to any spin-singlet and spin-triplet state, respectively.

\begin{tabular}{||c|c|c||}
\hline \multicolumn{3}{||c||}{ Table B2. Summary of dominant $K^{(*)} \rightarrow \eta K^{(*)}, \eta^{\prime} K^{(*)}$ transitions. } \\
\hline Transition type $\mathrm{A} \rightarrow \eta \mathrm{C}, \eta^{\prime} \mathrm{C}$ & even- $\mathrm{L}_{B C}$ dominant & odd- $\mathrm{L}_{B C}$ dominant \\
\hline \hline$K^{*} \rightarrow K$ & $\eta^{\prime} K$ & $\eta K$ \\
\hline$K^{*} \rightarrow K^{*}$ & $\eta K^{*}$ & $\eta^{\prime} K^{*}$ \\
\hline$K \rightarrow K^{*}$ & $\eta^{\prime} K^{*}$ & $\eta K^{*}$ \\
\hline
\end{tabular}

\section{B3.2 Application to $B$ decays}

Lipkin's rules for B decays, $B_{\eta^{\prime} K}>>B_{\eta K}$ but $B_{\eta K^{*}}>>B_{\eta^{\prime} K^{*}}$ 91, 115] follow from the first and third of our strong selection rules in Table B2. In this decay mechanism an initial $\mathrm{J}^{\mathrm{P}}=0^{-} n \bar{b} \mathrm{~B}$ meson is assumed to evolve weakly into a virtual $n \bar{s}$ system, which is a superposition of $\mathrm{J}^{\mathrm{P}}=0^{-}$and $0^{+}$states. This $\mathrm{J}=0$ intermediary then decays strongly into the observed $\eta^{\left({ }^{\prime}\right)} K^{(*)}$ final states. The $\eta K$ and $\eta^{\prime} K$ must be in S-wave since they arise from an initial $\mathrm{J}=0$ meson, and therefore have $\mathrm{J}^{\mathrm{P}}=0^{+}$, as does their excited $n \bar{s}$ precursor. This $\mathrm{J}^{\mathrm{P}}=0^{+} s \bar{n}$ precursor must be a spin-triplet. The first selection rule, for spin-triplet to spin-singlet transitions, " $K^{*}$ " $\rightarrow$ " $K$ ", says that for even- $\mathrm{L}_{B C}$ this transition is dominated by $\eta^{\prime} K$. In contrast the $\eta K^{*}$ and $\eta^{\prime} K^{*}$ final states must be in P-wave, since they also must have total $\mathrm{J}=0$. This $\mathrm{J}=0$ final state can only have $\mathrm{J}^{\mathrm{P}}=0^{-}$. The $\eta K^{*}$ and $\eta^{\prime} K^{*} s \bar{n}$ precursor thus has $\mathrm{J}^{\mathrm{P}}=0^{-}$, making it a spin-singlet. The third selection rule, for " $K$ " $\rightarrow$ " $K^{*}$ " transitions, states that in odd- $\mathrm{L}_{B C}$ this final state is dominated by $\eta K^{*}$. These two cases in combination agree with Lipkin [91]. Our second strong decay selection rule in Table B2, for " $K^{*}$ " $\rightarrow$ " $K^{*}$ ", is not accessed in B decays to $\eta^{(\prime)} K^{(*)}$ modes.

It is possible to test the second (" $K^{*}$ " $\rightarrow$ " $K^{*}$ ") selection rule in other B meson decays. Assuming an initial $\mathrm{J}^{\mathrm{P}}=0^{+}$spin-triplet source and a final state of an $\eta$ or $\eta^{\prime}$ combined with a spin-triplet kaon, it follows that the final kaon must have $\mathrm{J}=\mathrm{L}_{B C}$ and unnatural spin parity. The spin-triplet excited kaons accessible in " $K^{*}$ " $\rightarrow$ " $K^{*}$ " $\mathrm{B}$ decays therefore have $\mathrm{J}^{\mathrm{P}}=1^{+}, 2^{-}, 3^{+} \ldots$. Unfortunately these are just those kaon systems that experience singlet-triplet mixing, so we cannot observe a pure " $K^{*}$ " $\rightarrow$ " $K^{*}$ " transition in isolation. Experimentally we must instead study decays to singlet-triplet mixed states, in which both " $K^{*}$ " $\rightarrow$ " $K$ " and " $K^{*}$ " $\rightarrow$ " $K^{*}$ " transition amplitudes are present.

We may illustrate this using transitions to $\eta^{\left({ }^{\prime}\right)} K_{1}(1273)$ as an example. The decays $B \rightarrow \eta^{\left({ }^{\prime}\right)} K_{1}$ create a $\mathrm{P}$-wave (odd- $\left.\mathrm{L}_{B C}\right), \mathrm{J}^{\mathrm{P}}=0^{+}$final states, and hence are driven by an initial excited $\mathrm{J}^{\mathrm{P}}=0^{+}$ ${ }^{3} \mathrm{P}_{0}$ " $K^{*}$ " source. From Table B2 the " $K^{*} " \rightarrow$ " $K^{*}$ " amplitude, which couples the $B$ to the $\sin (\theta)\left|{ }^{3} \mathrm{P}_{1}\right\rangle$ " $K$ " component of the $K_{1}(1273)$, strongly favors the $\eta^{\prime}$. However the $\cos (\theta)\left|{ }^{1} \mathrm{P}_{1}\right\rangle$ " $K$ " component of the $K_{1}(1273)$ is also excited, through a " $K *$ " $\rightarrow$ " $K$ " transition, which in odd- $\mathrm{L}_{B C}$ dominantly favors the $\eta$. Thus the relative branching fraction $B_{B \rightarrow \eta^{\prime} K_{1}(1273) / \eta K_{1}(1273)}$ follows from a competition between these two processes, and is given by

$$
B_{B \rightarrow \eta^{\prime} K_{1}(1273) / \eta K_{1}(1273)}=\left|\frac{\sin (\theta) \mathcal{A}\left({ }^{3} \mathrm{P}_{0} \rightarrow{ }^{1} \mathrm{~S}_{0}+{ }^{3} \mathrm{P}_{1}\right)}{\cos (\theta) \mathcal{A}\left({ }^{3} \mathrm{P}_{0} \rightarrow{ }^{1} \mathrm{~S}_{0}+{ }^{1} \mathrm{P}_{1}\right)}\right|^{2}
$$


assuming complete dominance by the larger amplitudes in Table B2. Unfortunately these two amplitudes are different functions of momenta, so the $\tan ^{2}(\theta)$ state ratio is multiplied by a squared ratio of unknown transition amplitudes. A simplification is possible, since the corresponding result for the $K_{1}(1402)$ has $\sin (\theta)$ and $\cos (\theta)$ exchanged; if we take the ratio of relative branching fractions, we find a result that only involves the mixing angle $\theta$ (neglecting phase space differences between modes),

$$
\frac{B_{B \rightarrow \eta^{\prime} K_{1}(1273) / \eta K_{1}(1273)}}{B_{B \rightarrow \eta^{\prime} K_{1}(1402) / \eta K_{1}(1402)}}=\tan ^{4}(\theta) .
$$

For higher-mass states such as the $K_{2}$ kaons this "mixed" selection rule may thus allow an estimate of the corresponding singlet-triplet mixing angle, using the analogous $B \rightarrow \eta K_{2}$ and $\eta^{\prime} K_{2}$ decays.

\section{B3.3 Application to strong excited kaon decays}

To test these $\eta\left({ }^{\prime}\right) K^{*}$ strong selection rules directly in excited kaon decays we would ideally prefer a parent resonance with sufficient mass to populate both $\eta K^{*}$ and $\eta^{\prime} K^{*}$. This requires a mass above $M_{\eta^{\prime}}+M_{K^{*}}=1.90 \mathrm{GeV}$; of the excited kaons we have considered only the $1 \mathrm{~F}$ states satisfy this constraint. Since these $1 \mathrm{~F}$ states all have $\mathrm{P}=(+)$ they will populate even- $\mathrm{L}_{B C} \eta\left({ }^{\prime}\right) K^{*}$ final states. The $1^{3} \mathrm{~F}_{4}$ and $1^{3} \mathrm{~F}_{2}$ initial states are pure spin-triplet, which from Table B2 decay preferentially to $\eta K^{*}$ in this even- $\mathrm{L}_{B C}$ case. This is evident at the amplitude level in Table K14, with $\eta K^{*}$ preferred over $\eta^{\prime} K^{*}$ by an order of magnitude in the $1^{3} \mathrm{~F}_{2} K_{2}^{*}(2050)$ case. Unfortunately there are important centrifical barriers, especially in the decays of the $1^{3} \mathrm{~F}_{4} K_{2}^{*}(2045)$, which restrict the $\eta K^{*}$ branching fractions of these $1 \mathrm{~F}$ states to a few percent. A measurement of the ratio $B_{\eta K^{*}} / B_{\pi K^{*}}$ might be feasible for the $1^{3} \mathrm{~F}_{2} K_{2}^{*}(2050)$ state, once this resonance is identified.

Just as we found previously in considering $\eta K$ and $\eta^{\prime} K$ decays, it will be easier to study the strength of the lower mass mode $\eta K^{*}$, since the lower threshold of $\approx 1.44 \mathrm{GeV}$ makes more resonance couplings accessible. The ratio $B_{\eta K^{*}} / B_{\pi K^{*}}$ can provide a normalized measure of the strength of the $\eta K^{*}$ mode. Of the states we have considered, the single spin-triplet excited kaon below $\eta^{\prime} K^{*}$ threshold that is expected to have a large $\eta K^{*}$ branching fraction is the as yet unidentified $2^{3} \mathrm{P}_{2}$ $K_{2}^{*}(1850)$; this has a theoretical branching fraction of $B_{\eta K^{*}}=7 \%$, and a branching fraction ratio of $B_{\eta K^{*}} / B_{\pi K^{*}}=0.55$. The remaining interesting excited kaon states below $\eta^{\prime} K^{*}$ threshold are the known $1^{3} \mathrm{D}_{3} K_{3}^{*}(1776)$ and $1^{3} \mathrm{D}_{1} K^{*}(1717)$; these have suppressed $\eta K^{*}$ modes since they are odd- $\mathrm{L}_{B C}$, and their $\eta K^{*}$ branching fractions are predicted to be smaller than the corresponding $\pi K^{*}$ branching fractions by factors of about 160 (for $K_{3}^{*}(1776)$ ) and 50 (for $K^{*}(1717)$ ) respectively.

Decay amplitudes of the mixed singlet-triplet mesons to these modes are quite sensitive to the singlet-triplet mixing angles, and may be useful in determining these parameters more accurately; even in the relatively well-studied $1 \mathrm{P}$ case $\left(K_{1}(1273)\right.$ and $\left.K_{1}(1402)\right)$ the mixing angle is not well determined by the existing decay measurements (see Fig.4). Since the mixed-spin kaons all have unnatural $\mathrm{J}^{\mathrm{P}}$, the lightest final state of relevance here is $\eta K^{*}$, which can be used to estimate the singlet-triplet mixing for example in the expected $2^{1} \mathrm{P}_{1}-2^{3} \mathrm{P}_{1} K_{1}(1800)$ and $1^{1} \mathrm{~F}_{3}-1^{3} \mathrm{~F}_{3} K_{3}(2050)$ pairs. The spin-triplet component in each of these resonances strongly favors $\eta K^{*}$, as shown in Tables K10 and K15. 


\begin{tabular}{||c||c|c||}
\hline \multicolumn{3}{||c||}{ Table S1. 1S $s \bar{s}$} \\
\hline \multicolumn{1}{||c||}{} & \multicolumn{2}{c||}{$\phi(1019)$} \\
\hline Mode & $\Gamma_{i}(\mathrm{MeV})$ & Amps. \\
\hline \hline$K K$ & 2.5 & ${ }^{1} P_{1}=-0.081$ \\
\hline \hline \multicolumn{1}{||c||}{} & \multicolumn{2}{|c||}{$\Gamma_{\text {thy }}=2.5 \mathrm{MeV}$} \\
\hline & $\Gamma_{\text {expt }}=4.26 \pm 0.05 \mathrm{MeV}$ \\
\hline
\end{tabular}

\begin{tabular}{|c|c|c|c|c|}
\hline \multicolumn{5}{|c|}{ Table S2. 2S s $\bar{s}$} \\
\hline & \multicolumn{2}{|c|}{$\phi(1680)$} & \multicolumn{2}{|c|}{$\eta_{s}(1415 \rightarrow 1500)$} \\
\hline Mode & $\overline{\Gamma_{i}(\mathrm{MeV})}$ & Amps. & $\overline{\Gamma_{i}(\mathrm{MeV})}$ & Amps. \\
\hline$\overline{K K K}$ & 89 & ${ }^{1} P_{1}=-0.16$ & 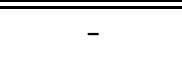 & 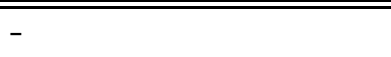 \\
\hline$K K^{*}$ & 245 & ${ }^{3} P_{1}=-0.23$ & $11 \rightarrow 100$ & ${ }^{3} P_{0}=+0.10 \rightarrow+0.20$ \\
\hline$\overline{\eta \eta \phi}$ & $\overline{44}$ & ${ }^{3} P_{1}=+0.25$ & - & - \\
\hline & \multicolumn{2}{|c|}{$\overline{\Gamma_{t h y}}=378 \mathrm{MeV}$} & \multicolumn{2}{|c|}{ 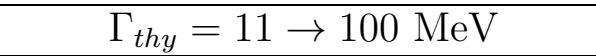 } \\
\hline & \multicolumn{2}{|c|}{$\Gamma_{\text {expt }}=150 \pm 50 \mathrm{MeV}$} & \multicolumn{2}{|c|}{$\Gamma_{\text {expt }}(\eta(1440)) \approx 50-80 \mathrm{MeV}$} \\
\hline
\end{tabular}

\begin{tabular}{|c|c|c|c|c|}
\hline \multicolumn{5}{|c|}{ Table S3. 3S $s \bar{s}$} \\
\hline & \multicolumn{2}{|c|}{$\phi(2050)$} & \multicolumn{2}{|c|}{$\eta_{s}(1950)$} \\
\hline Mode & $\overline{\Gamma_{i}(\mathrm{MeV})}$ & Amps. & $\Gamma_{i}(\mathrm{MeV})$ & Amps. \\
\hline$\overline{K K K}$ & 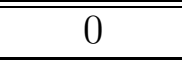 & ${ }^{1} P_{1}=+0.0029$ & - & - \\
\hline$K K^{*}$ & 20 & ${ }^{3} P_{1}=-0.047$ & 53 & ${ }^{3} P_{0}=+0.081$ \\
\hline$K^{*} K^{*}$ & 102 & $\begin{array}{l}{ }^{1} P_{1}=-0.039 \\
{ }^{5} P_{1}=+0.17\end{array}$ & 67 & ${ }^{3} P_{0}=-0.17$ \\
\hline$K K_{1}(1273)$ & 58 & $\begin{array}{l}{ }^{3} S_{1} \equiv 0 \\
{ }^{3} D_{1}=-0.10\end{array}$ & - & - \\
\hline$K K_{1}(1402)$ & 26 & $\begin{array}{l}{ }^{3} S_{1}=+0.083 \\
{ }^{3} D_{1} \equiv 0\end{array}$ & - & - \\
\hline$K K_{0}^{*}(1412)$ & - & - & 30 & ${ }^{1} S_{0}=-0.13$ \\
\hline$K K_{2}^{*}(1429)$ & 9 & ${ }^{5} D_{1}=+0.053$ & 0 & ${ }^{5} D_{0}=-0.016$ \\
\hline$K K^{*}(1414)$ & 93 & ${ }^{3} P_{1}=-0.16$ & 25 & ${ }^{3} P_{0}=+0.12$ \\
\hline$K K(1460)$ & 29 & ${ }^{1} P_{1}=-0.10$ & - & - \\
\hline$\overline{\eta \eta \phi}$ & 21 & ${ }^{3} P_{1}=+0.10$ & 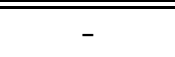 & 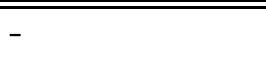 \\
\hline$\eta^{\prime} \phi$ & 11 & ${ }^{3} P_{1}=-0.11$ & - & - \\
\hline$\eta h_{1}(1386)$ & 8 & $\begin{array}{l}{ }^{3} S_{1}=-0.078 \\
{ }^{3} D_{1}=-0.060\end{array}$ & - & - \\
\hline & \multicolumn{2}{|c|}{$\overline{\Gamma_{\text {thy }}=378 \mathrm{MeV}}$} & \multicolumn{2}{|c|}{ 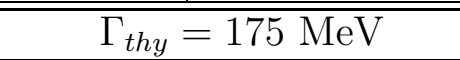 } \\
\hline
\end{tabular}




\begin{tabular}{||c||c|c||c|c||}
\hline \multicolumn{5}{||c||}{ Table S4. $1^{3} \mathrm{P}_{2}$ and $1^{3} \mathrm{P}_{0} s \bar{s}$} \\
\hline Mode & $\Gamma_{i}(\mathrm{MeV})$ & Amps. & $\Gamma_{i}(\mathrm{MeV})$ & Amps. \\
\hline \hline$K K$ & 61 & ${ }^{1} D_{2}=+0.15$ & 214 & ${ }^{1} S_{0}=+0.28$ \\
\hline$K K^{*}$ & 9 & ${ }^{3} D_{2}=+0.056$ & - & - \\
\hline \hline$\eta \eta$ & 10 & ${ }^{1} D_{2}=-0.13$ & 66 & ${ }^{1} S_{0}=-0.33$ \\
\hline$\eta \eta^{\prime}$ & 0 & ${ }^{1} D_{2}=+0.0073$ & - & - \\
\hline \hline \multicolumn{1}{||c||}{} & \multicolumn{3}{|c||}{$\Gamma_{\text {thy }}=80 \mathrm{MeV}$} & \multicolumn{2}{c||}{$\Gamma_{\text {thy }}=279 \mathrm{MeV}$} \\
\hline & \multicolumn{1}{|c||}{$\Gamma_{\text {expt }}=76 \pm 10 \mathrm{MeV}$} & \multicolumn{2}{c||}{ see text } \\
\hline
\end{tabular}

\begin{tabular}{||c||c|c||}
\hline \multicolumn{3}{||c||}{ Table S5. $1^{3} \mathrm{P}_{1} s \bar{s}$} \\
\hline & \multicolumn{2}{|c||}{$f_{1}(1420 \rightarrow 1530)$} \\
\hline Mode & $\Gamma_{i}(\mathrm{MeV})$ & Amps. \\
\hline \hline \multirow{2}{*}{$K K^{*}$} & \multirow{2}{*}{$254 \rightarrow 459$} & $\begin{array}{l}{ }^{3} S_{1}=-0.47 \rightarrow-0.40 \\
{ }^{3} D_{1}=+0.0092 \rightarrow+0.043\end{array}$ \\
\hline \hline & \multicolumn{2}{||}{$\Gamma_{\text {thy }}=254 \rightarrow 459 \mathrm{MeV}$} \\
\hline & \multicolumn{2}{c||}{ see text } \\
\hline
\end{tabular}

\begin{tabular}{||c||c|c||}
\hline \multicolumn{1}{||c||}{ Table S6. $1^{1} \mathrm{P}_{1} s \bar{s}$} \\
\hline \multicolumn{1}{||c||}{$h_{1}(1390 \rightarrow 1440)$} \\
\hline Mode & $\Gamma_{i}(\mathrm{MeV})$ & Amps. \\
\hline \hline \multirow{2}{*}{$K K^{*}$} & $0 \rightarrow 160$ & $\begin{array}{l}{ }^{3} S_{1}=-0.34 \rightarrow-0.32 \\
{ }^{3} D_{1}=0 \rightarrow-0.022\end{array}$ \\
\hline \hline & \multicolumn{2}{|c||}{$\Gamma_{\text {thy }}=0 \rightarrow 160 \mathrm{MeV}$} \\
\hline & \multicolumn{2}{|c||}{$\Gamma_{\text {expt }}=91 \pm 30 \mathrm{MeV}$} \\
\hline
\end{tabular}




\begin{tabular}{|c|c|c|c|c|c|c|}
\hline \multicolumn{7}{|c|}{ Table S7. $2^{3} \mathrm{P}_{\mathrm{J}} s \bar{s}$} \\
\hline & \multicolumn{2}{|r|}{$f_{2}(2000)$} & \multicolumn{2}{|r|}{$f_{1}(1950)$} & \multicolumn{2}{|c|}{$f_{0}(2000)$} \\
\hline Mode & $\Gamma_{i}(\mathrm{MeV})$ & Amps. & $\Gamma_{i}(\mathrm{MeV})$ & Amps. & $\overline{\Gamma_{i}(\mathrm{MeV})}$ & Amps. \\
\hline 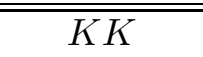 & 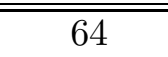 & ${ }^{1} D_{2}=+0.11$ & - & 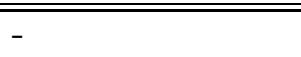 & 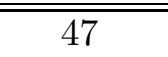 & 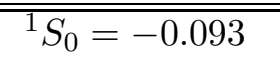 \\
\hline$K K^{*}$ & 142 & ${ }^{3} D_{2}=+0.13$ & 68 & $\begin{array}{l}{ }^{3} S_{1}=+0.0025 \\
{ }^{3} D_{1}=+0.092\end{array}$ & - & - \\
\hline$K^{*} K^{*}$ & 101 & $\begin{array}{l}{ }^{5} S_{2}=-0.16 \\
{ }^{1} D_{2}=+0.036 \\
{ }^{5} D_{2}=-0.094\end{array}$ & 29 & ${ }^{5} D_{1}=-0.11$ & 89 & $\begin{array}{l}{ }^{1} S_{0}=+0.080 \\
{ }^{5} D_{0}=-0.16\end{array}$ \\
\hline$K K_{1}(1273)$ & 14 & $\begin{array}{l}{ }^{3} P_{2}=+0.045 \\
{ }^{3} F_{2}=+0.032\end{array}$ & 108 & ${ }^{3} P_{1}=-0.16$ & 423 & ${ }^{3} P_{0}=+0.30$ \\
\hline$K K_{1}(1402)$ & 21 & $\begin{array}{l}{ }^{3} P_{2}=-0.086 \\
{ }^{3} F_{2} \equiv 0\end{array}$ & 1 & ${ }^{3} P_{1}=-0.025$ & 0 & ${ }^{3} P_{0}=-0.0048$ \\
\hline$K K_{0}^{*}(1412)$ & - & - & 1 & ${ }^{1} P_{1}=-0.026$ & - & - \\
\hline$K K_{2}^{*}(1429)$ & 21 & $\begin{array}{l}{ }^{5} P_{2}=-0.093 \\
{ }^{5} F_{2}=-0.0050\end{array}$ & 8 & $\begin{array}{l}{ }^{5} P_{1}=+0.081 \\
{ }^{5} F_{1}=-0.00087\end{array}$ & - & - \\
\hline$K K^{*}(1414)$ & 4 & ${ }^{3} D_{2}=+0.039$ & 80 & $\begin{array}{l}{ }^{3} S_{1}=-0.22 \\
{ }^{3} D_{1}=+0.013\end{array}$ & - & - \\
\hline$K K(1460)$ & 0 & ${ }^{1} D_{2}=+0.016$ & - & - & 125 & ${ }^{1} S_{0}=+0.26$ \\
\hline$\overline{\eta \eta}$ & 16 & ${ }^{1} D_{2}=-0.11$ & 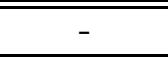 & 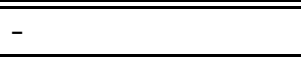 & 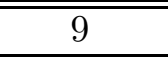 & ${ }^{1} S_{0}=+0.082$ \\
\hline$\eta \eta^{\prime}$ & 19 & ${ }^{1} D_{2}=+0.097$ & - & - & 0 & ${ }^{1} S_{0}=+0.012$ \\
\hline$\eta^{\prime} \eta^{\prime}$ & 0 & ${ }^{1} D_{2}=-0.029$ & - & - & 25 & ${ }^{1} S_{0}=-0.24$ \\
\hline$\eta \eta_{s}(1415)$ & 0 & ${ }^{1} D_{2}=-0.021$ & - & - & 64 & ${ }^{1} S_{0}=-0.38$ \\
\hline & \multicolumn{2}{|c|}{ 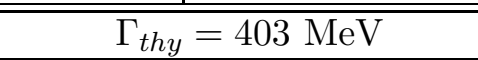 } & \multicolumn{2}{|c|}{$\overline{\Gamma_{t h y}}=296 \mathrm{MeV}$} & \multicolumn{2}{|c|}{$\bar{\Gamma}_{t h y}=782 \mathrm{MeV}$} \\
\hline
\end{tabular}

\begin{tabular}{|c|c|c|}
\hline \multicolumn{3}{|c|}{ Table S8. $2{ }^{1} \mathrm{P}_{1} s \bar{s}$} \\
\hline & \multicolumn{2}{|c|}{$h_{1}(1850)$} \\
\hline Mode & $\overline{\Gamma_{i}(\mathrm{MeV})}$ & Amps. \\
\hline$K K^{*}$ & 95 & $\begin{array}{l}{ }^{3} S_{1}=-0.024 \\
{ }^{3} D_{1}=-0.12\end{array}$ \\
\hline$K^{*} K^{*}$ & 64 & $\begin{array}{l}{ }^{3} S_{1}=+0.21 \\
{ }^{3} D_{1}=+0.037\end{array}$ \\
\hline$K K_{1}(1273)$ & 1 & ${ }^{3} P_{1}=+0.024$ \\
\hline$\eta \phi$ & 33 & $\begin{array}{l}{ }^{3} S_{1}=+0.098 \\
{ }^{3} D_{1}=+0.12\end{array}$ \\
\hline & \multicolumn{2}{|c|}{$\Gamma_{t h y}=193 \mathrm{MeV}$} \\
\hline
\end{tabular}




\begin{tabular}{|c|c|c|c|c|c|c|}
\hline \multicolumn{7}{|c|}{ Table S9. $1^{3} \mathrm{D}_{\mathrm{J}} s \bar{s}$} \\
\hline & \multicolumn{2}{|r|}{$\phi_{3}(1854)$} & \multicolumn{2}{|c|}{$\phi_{2}(1850)$} & \multicolumn{2}{|c|}{$\phi(1850)$} \\
\hline Mode & $\Gamma_{i}(\mathrm{MeV})$ & Amps. & $\Gamma_{i}(\mathrm{MeV})$ & Amps. & $\overline{\Gamma_{i}(\mathrm{MeV})}$ & Amps. \\
\hline 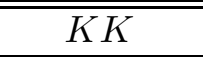 & 45 & ${ }^{1} F_{3}=-0.10$ & 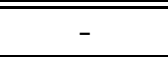 & 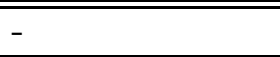 & 65 & ${ }^{1} P_{1}=-0.12$ \\
\hline$K K^{*}$ & 24 & ${ }^{3} F_{3}=-0.059$ & 151 & $\begin{array}{l}{ }^{3} P_{2}=+0.14 \\
{ }^{3} F_{2}=-0.049\end{array}$ & 75 & ${ }^{3} P_{1}=+0.11$ \\
\hline$K^{*} K^{*}$ & 32 & $\begin{array}{l}{ }^{5} P_{3}=+0.15 \\
{ }^{1} F_{3}=-0.0024 \\
{ }^{5} F_{3}=+0.0052 \\
{ }^{5} H_{3} \equiv 0\end{array}$ & 7 & $\begin{array}{l}{ }^{5} P_{2}=+0.073 \\
{ }^{5} F_{2}=+0.0072\end{array}$ & 5 & $\begin{array}{l}{ }^{1} P_{1}=-0.054 \\
{ }^{5} P_{1}=+0.024 \\
{ }^{5} F_{1}=+0.0088\end{array}$ \\
\hline$K K_{1}(1273)$ & 0 & $\begin{array}{l}{ }^{3} D_{3}=-0.012 \\
{ }^{3} G_{3}=-0.00061\end{array}$ & 2 & ${ }^{3} D_{2}=+0.028$ & 478 & $\begin{array}{l}{ }^{3} S_{1}=-0.45 \\
{ }^{3} D_{1}=-0.019\end{array}$ \\
\hline$\eta \phi$ & 3 & ${ }^{3} F_{3}=+0.046$ & 53 & $\begin{array}{l}{ }^{3} P_{2}=-0.20 \\
{ }^{3} F_{2}=+0.038\end{array}$ & 29 & ${ }^{3} P_{1}=-0.15$ \\
\hline & \multicolumn{2}{|c|}{$\overline{\Gamma_{t h y}}=104 \mathrm{MeV}$} & \multicolumn{2}{|c|}{$\overline{\Gamma_{t h y}}=214 \mathrm{MeV}$} & \multicolumn{2}{|c|}{$\overline{\Gamma_{t h y}}=652 \mathrm{MeV}$} \\
\hline & \multicolumn{2}{|c|}{$\Gamma_{\text {expt }}=87_{-23}^{+28} \mathrm{MeV}$} & & & & \\
\hline
\end{tabular}

\begin{tabular}{||c||c|c||}
\hline \multicolumn{3}{||c||}{ Table S10. $1^{1} \mathrm{D}_{2} s \bar{s}$} \\
\hline \multicolumn{1}{||c||}{} & \multicolumn{2}{c||}{$\eta_{2}(1850)$} \\
\hline \hline$K K^{*}$ & 114 & $\begin{array}{l}{ }^{3} P_{2}=+0.12 \\
{ }^{3} F_{2}=+0.060\end{array}$ \\
\hline$K^{*} K^{*}$ & 15 & $\begin{array}{l}{ }^{3} P_{2}=-0.10 \\
{ }^{3} F_{2}=-0.0062\end{array}$ \\
\hline$K K_{1}(1273)$ & 0 & ${ }^{3} D_{2}=-0.011$ \\
\hline \hline & \multicolumn{2}{|c||}{$\Gamma_{t h y}=129 \mathrm{MeV}$} \\
\hline
\end{tabular}




\begin{tabular}{|c|c|c|}
\hline \multicolumn{3}{|c|}{ Table S11. $1^{1} \mathrm{D}_{2}$ (alt.) } \\
\hline & \multicolumn{2}{|c|}{$\overline{\eta_{2}(1842)=s|n \bar{n}\rangle+c|s \bar{s}\rangle}$} \\
\hline Mode & $\Gamma_{i}(\mathrm{MeV})$ & Amps. \\
\hline$K K^{*}$ & $111.7 c^{2}+158.0 s c+55.9 s^{2}$ & $\begin{array}{l}{ }^{3} P_{2}=-0.116 c-0.0820 s \\
{ }^{3} F_{2}=-0.0580 c-0.0410 s\end{array}$ \\
\hline$K^{*} K^{*}$ & $12.1 c^{2}-17.1 s c+6.1 s^{2}$ & $\begin{array}{l}{ }^{3} P_{2}=-0.10 \\
{ }^{3} F_{2}=+0.00506 c-0.00358 s\end{array}$ \\
\hline$K K_{1}(1273)$ & $0.2 c^{2}-0.3 s c+0.1 s^{2}$ & ${ }^{3} D_{2}=+0.00977 c-0.00691 s$ \\
\hline$\rho \rho$ & $129.9 s^{2}$ & $\begin{array}{l}{ }^{3} P_{2}=+0.231 s \\
{ }^{3} F_{2}=-0.0765 s\end{array}$ \\
\hline$\omega \omega$ & $40.3 s^{2}$ & $\begin{array}{l}{ }^{3} P_{2}=-0.229 s \\
{ }^{3} F_{2}=-0.0689 s\end{array}$ \\
\hline$\pi a_{1}$ & $33.8 s^{2}$ & ${ }^{3} D_{2}=+0.0986 s$ \\
\hline$\eta f_{1}$ & 0.0 & ${ }^{3} D_{2}=-0.00321 s$ \\
\hline$\pi a_{0}(1450)$ & $0.6 s^{2}$ & ${ }^{1} D_{2}=+0.0182 s$ \\
\hline$\pi a_{2}$ & $260.7 s^{2}$ & $\begin{array}{l}{ }^{5} S_{2}=+0.285 s \\
{ }^{5} D_{2}=+0.109 s \\
{ }^{5} G_{2}=+0.00681 s\end{array}$ \\
\hline$\eta f_{2}$ & $30.0 s^{2}$ & $\begin{array}{l}{ }^{5} S_{2}=-0.314 s \\
{ }^{5} D_{2}=-0.00721 s \\
{ }^{5} G_{2}=-0.0000358 s\end{array}$ \\
\hline & \multicolumn{2}{|c|}{ 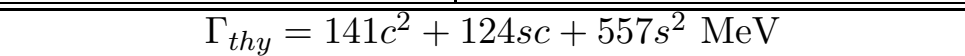 } \\
\hline & \multicolumn{2}{|c|}{$\Gamma_{\text {expt }}=225 \pm 14 \mathrm{MeV}$} \\
\hline
\end{tabular}

\begin{tabular}{|c|c|c|}
\hline \multicolumn{3}{|c|}{ Table S12. $1^{1} \mathrm{D}_{2}$ partner (alt.) } \\
\hline & \multicolumn{2}{|c|}{$\eta_{2}(1617)=c|n \bar{n}\rangle-s|s \bar{s}\rangle$} \\
\hline Mode & $\Gamma_{i}(\mathrm{MeV})$ & Amps. \\
\hline$K K^{*}$ & $22.6 c^{2}-63.8 s c+45.1 s^{2}$ & $\begin{array}{l}{ }^{3} P_{2}=-0.0749 c+0.106 s \\
{ }^{3} F_{2}=-0.0141 c+0.0200 s\end{array}$ \\
\hline$\rho \rho$ & $21.4 c^{2}$ & $\begin{array}{l}{ }^{3} P_{2}=+0.150 c \\
{ }^{3} F_{2}=+0.00983 c\end{array}$ \\
\hline$\omega \omega$ & $6.2 c^{2}$ & $\begin{array}{l}{ }^{3} P_{2}=-0.129 c \\
{ }^{3} F_{2}=-0.00573 c\end{array}$ \\
\hline$\pi a_{1}$ & $3.1 c^{2}$ & ${ }^{3} D_{2}=+0.0436 c$ \\
\hline$\pi a_{0}(1450)$ & 0.0 & ${ }^{1} D_{2}=+0.00158 c$ \\
\hline$\pi a_{2}$ & $169.4 c^{2}$ & $\begin{array}{l}{ }^{5} S_{2}=+0.402 c \\
{ }^{5} D_{2}=+0.0369 c \\
{ }^{5} G_{2}=+0.000692 c\end{array}$ \\
\hline & \multicolumn{2}{|c|}{$\overline{\Gamma_{t h y}=221 c^{2}-64 s c+45 s^{2} \mathrm{MeV}}$} \\
\hline & \multicolumn{2}{|c|}{$\Gamma_{\text {expt }}=181 \pm 11 \mathrm{MeV}$} \\
\hline
\end{tabular}




\begin{tabular}{|c|c|c|c|c|c|c|}
\hline \multicolumn{7}{|c|}{ Table S13. $1^{3} \mathrm{~F}_{\mathrm{J}} s \bar{s}$} \\
\hline & \multicolumn{2}{|r|}{$f_{4}(2200)$} & \multicolumn{2}{|c|}{$f_{3}(2200)$} & \multicolumn{2}{|r|}{$f_{2}(2200)$} \\
\hline Mode & $\Gamma_{i}^{(\mathrm{MeV})}$ & Amps. & $\Gamma_{i}^{(\mathrm{MeV})}$ & Amps. & $\Gamma_{i}^{(\mathrm{MeV})}$ & Amps. \\
\hline 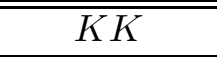 & 36 & ${ }^{1} G_{4}=+0.073$ & - & - & 27 & ${ }^{1} D_{2}=+0.063$ \\
\hline$K K^{*}$ & 28 & ${ }^{3} G_{4}=+0.050$ & 78 & $\begin{array}{l}{ }^{3} D_{3}=-0.070 \\
{ }^{3} G_{3}=+0.044\end{array}$ & 39 & ${ }^{3} D_{2}=-0.059$ \\
\hline$K^{*} K^{*}$ & 53 & $\begin{array}{l}{ }^{5} D_{4}=-0.11 \\
{ }^{1} G_{4}=+0.010 \\
{ }^{5} G_{4}=-0.020 \\
{ }^{5} I_{4} \equiv 0\end{array}$ & 21 & $\begin{array}{l}{ }^{5} D_{3}=-0.062 \\
{ }^{5} G_{3}=-0.032\end{array}$ & 16 & $\begin{array}{l}{ }^{5} S_{2} \equiv 0 \\
{ }^{1} D_{2}=+0.037 \\
{ }^{5} D_{2}=-0.028 \\
{ }^{5} G_{2}=-0.040\end{array}$ \\
\hline$K K_{1}(1273)$ & 5 & $\begin{array}{l}{ }^{3} F_{4}=+0.024 \\
{ }^{3} H_{4}=+0.0049\end{array}$ & 18 & ${ }^{3} F_{3}=-0.048$ & 243 & $\begin{array}{l}{ }^{3} P_{2}=+0.18 \\
{ }^{3} F_{2}=+0.023\end{array}$ \\
\hline$K K_{1}(1402)$ & 6 & $\begin{array}{l}{ }^{3} F_{4}=-0.031 \\
{ }^{3} H_{4} \equiv 0\end{array}$ & 0 & ${ }^{3} F_{3}=-0.0089$ & 0 & $\begin{array}{l}{ }^{3} P_{2} \equiv 0 \\
{ }^{3} F_{2}=+0.0074\end{array}$ \\
\hline$K K_{0}^{*}(1412)$ & - & - & 2 & ${ }^{1} F_{3}=-0.018$ & - & - \\
\hline$K K_{2}^{*}(1429)$ & 5 & $\begin{array}{l}{ }^{5} F_{4}=-0.029 \\
{ }^{5} H_{4}=-0.0011\end{array}$ & 120 & $\begin{array}{l}{ }^{5} P_{3}=+0.14 \\
{ }^{5} F_{3}=-0.00048 \\
{ }^{5} H_{3}=-0.0013\end{array}$ & 22 & $\begin{array}{l}{ }^{5} P_{2}=+0.060 \\
{ }^{5} F_{2}=+0.015\end{array}$ \\
\hline$K K^{*}(1414)$ & 0 & ${ }^{3} G_{4}=+0.0081$ & 1 & $\begin{array}{l}{ }^{3} D_{3}=-0.012 \\
{ }^{3} G_{3}=+0.0072\end{array}$ & 1 & ${ }^{3} D_{2}=-0.0098$ \\
\hline$K K(1460)$ & 0 & ${ }^{1} G_{4}=+0.0052$ & - & - & 1 & ${ }^{1} D_{2}=+0.012$ \\
\hline$K^{*} K_{1}(1273)$ & 0 & $\begin{array}{l}{ }^{3} F_{4}=+0.00031 \\
{ }^{5} F_{4}=-0.0012 \\
{ }^{3} H_{4}=-0.000011 \\
{ }^{5} H_{4}=-0.000014\end{array}$ & 23 & $\begin{array}{l}{ }^{5} P_{3}=+0.096 \\
{ }^{1} F_{3}=-0.00087 \\
{ }^{3} F_{3}=+0.00091 \\
{ }^{5} F_{3}=+0.0012 \\
{ }^{5} H_{3}=-0.000015\end{array}$ & 16 & $\begin{array}{l}{ }^{3} P_{2}=-0.069 \\
{ }^{5} P_{2}=+0.040 \\
{ }^{3} F_{2}=-0.0018 \\
{ }^{5} F_{2}=+0.0022\end{array}$ \\
\hline 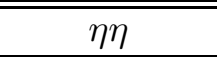 & 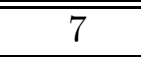 & ${ }^{1} G_{4}=-0.067$ & - & 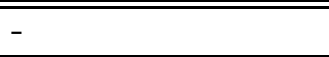 & 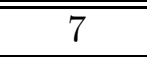 & ${ }^{1} D_{2}=-0.066$ \\
\hline$\eta \eta^{\prime}$ & 4 & ${ }^{1} G_{4}=+0.036$ & - & - & 14 & ${ }^{1} D_{2}=+0.072$ \\
\hline$\eta^{\prime} \eta^{\prime}$ & 0 & ${ }^{1} G_{4}=-0.0095$ & - & - & 3 & ${ }^{1} D_{2}=-0.053$ \\
\hline$\eta f_{1}^{s \bar{s}}(1426)$ & 0 & $\begin{array}{l}{ }^{3} F_{4}=+0.017 \\
{ }^{3} H_{4}=-0.00091\end{array}$ & 1 & ${ }^{3} F_{3}=+0.025$ & 29 & $\begin{array}{l}{ }^{3} P_{2}=-0.15 \\
{ }^{3} F_{2}=-0.014\end{array}$ \\
\hline$\eta f_{0}^{s \bar{s}}(1500)$ & - & - & 0 & ${ }^{1} F_{3}=+0.010$ & - & - \\
\hline$\eta f_{2}^{\prime}(1525)$ & 0 & $\begin{array}{l}{ }^{5} F_{4}=+0.014 \\
{ }^{5} H_{4}=+0.00026\end{array}$ & 28 & $\begin{array}{l}{ }^{5} P_{3}=-0.17 \\
{ }^{5} F_{3}=+0.00011 \\
{ }^{5} H_{3}=+0.00030\end{array}$ & 5 & $\begin{array}{l}{ }^{5} P_{2}=-0.073 \\
{ }^{5} F_{2}=-0.0078\end{array}$ \\
\hline$\eta \eta_{s}(1415)$ & 0 & ${ }^{1} G_{4}=-0.0076$ & - & - & 0 & ${ }^{1} D_{2}=-0.017$ \\
\hline$\phi \phi$ & 10 & $\begin{array}{l}{ }^{5} D_{4}=+0.12 \\
{ }^{1} G_{4}=-0.0040 \\
{ }^{5} G_{4}=+0.0080 \\
{ }^{5} I_{4} \equiv 0\end{array}$ & 4 & $\begin{array}{l}{ }^{5} D_{3}=+0.070 \\
{ }^{5} G_{3}=+0.013\end{array}$ & 2 & $\begin{array}{l}{ }^{5} S_{2} \equiv 0 \\
{ }^{1} D_{2}=-0.041 \\
{ }^{5} D_{2}=+0.031 \\
{ }^{5} G_{2}=+0.016\end{array}$ \\
\hline & & hey = $156 \mathrm{MeV}$ & & $2 y=297 \mathrm{MeV}$ & & $=425 \mathrm{MeV}$ \\
\hline
\end{tabular}




\begin{tabular}{|c|c|c|}
\hline \multicolumn{3}{|c|}{ Table S14. $1^{1} \mathrm{~F}_{3} s \bar{s}$} \\
\hline & \multicolumn{2}{|r|}{$h_{3}(2200)$} \\
\hline Mode & $\overline{\Gamma_{i}(\mathrm{MeV})}$ & Amps. \\
\hline$K K^{*}$ & 71 & $\begin{array}{l}{ }^{3} D_{3}=-0.061 \\
{ }^{3} G_{3}=-0.050\end{array}$ \\
\hline$K^{*} K^{*}$ & 29 & $\begin{array}{l}{ }^{3} D_{3}=+0.076 \\
{ }^{3} G_{3}=+0.028\end{array}$ \\
\hline$K K_{1}(1273)$ & 4 & ${ }^{3} F_{3}=+0.022$ \\
\hline$K K_{1}(1402)$ & 2 & ${ }^{3} F_{3}=+0.019$ \\
\hline$K K_{0}^{*}(1412)$ & 1 & ${ }^{1} F_{3}=+0.014$ \\
\hline$K K_{2}^{*}(1429)$ & 94 & $\begin{array}{l}{ }^{5} P_{3}=+0.13 \\
{ }^{5} F_{3}=+0.025 \\
{ }^{5} H_{3}=+0.0015\end{array}$ \\
\hline$K K^{*}(1414)$ & 1 & $\begin{array}{l}{ }^{3} D_{3}=-0.010 \\
{ }^{3} G_{3}=-0.0083\end{array}$ \\
\hline$K^{*} K_{1}(1273)$ & 17 & $\begin{array}{l}{ }^{5} P_{3}=-0.083 \\
{ }^{1} F_{3} \equiv 0 \\
{ }^{3} F_{3}=+0.00053 \\
{ }^{5} F_{3}=-0.0020 \\
{ }^{5} H_{3}=-0.000018\end{array}$ \\
\hline$\eta \phi$ & 25 & $\begin{array}{l}{ }^{3} D_{3}=+0.085 \\
{ }^{3} G_{3}=+0.050\end{array}$ \\
\hline$\eta^{\prime} \phi$ & 5 & $\begin{array}{l}{ }^{3} D_{3}=-0.054 \\
{ }^{3} G_{3}=-0.010\end{array}$ \\
\hline$\eta h_{1}(1386)$ & 0 & ${ }^{3} F_{3} \equiv 0$ \\
\hline & & $=249 \mathrm{MeV}$ \\
\hline
\end{tabular}




\begin{tabular}{||c||c|c||}
\hline \multicolumn{3}{||c||}{ Table K1. 1S kaons } \\
\hline & \multicolumn{2}{|c||}{$K^{*}(894)$} \\
\hline Mode & $\Gamma_{i}(\mathrm{MeV})$ & Amps. \\
\hline \hline$\pi K$ & 21 & ${ }^{1} P_{1}=+0.14$ \\
\hline \hline & \multicolumn{2}{|c||}{$\Gamma_{\text {thy }}=21 \mathrm{MeV}$} \\
\hline & \multicolumn{2}{|c||}{$\Gamma_{\text {expt }}=51(1) \mathrm{MeV}$} \\
\hline
\end{tabular}

\begin{tabular}{|c|c|c|c|c|}
\hline \multicolumn{5}{|c|}{ Table K2. 2S kaons } \\
\hline & \multicolumn{2}{|r|}{$K^{*}(1414)$} & \multicolumn{2}{|c|}{$K(1460)$} \\
\hline Mode & $\Gamma_{i}(\mathrm{MeV})$ & Amps. & $\overline{\Gamma_{i}(\mathrm{MeV})}$ & Amps. \\
\hline$\overline{\pi k K}$ & $\overline{55}$ & ${ }^{1} P_{1}=+0.12$ & - & - \\
\hline$\eta K$ & 42 & ${ }^{1} P_{1}=+0.20$ & - & - \\
\hline$\rho K$ & 34 & ${ }^{3} P_{1}=+0.13$ & 73 & ${ }^{3} P_{0}=-0.18$ \\
\hline$\omega K$ & 10 & ${ }^{3} P_{1}=+0.13$ & 23 & ${ }^{3} P_{0}=-0.17$ \\
\hline$\pi K^{*}$ & 55 & ${ }^{3} P_{1}=-0.15$ & 101 & ${ }^{3} P_{0}=-0.20$ \\
\hline$\eta K^{*}$ & - & - & 3 & ${ }^{3} P_{0}=-0.11$ \\
\hline$\pi K_{1}(1273)$ & 0.0 & $\begin{array}{l}{ }^{3} S_{1} \equiv 0 \\
{ }^{3} D_{1}=+0.00039\end{array}$ & - & - \\
\hline & \multicolumn{2}{|c|}{ 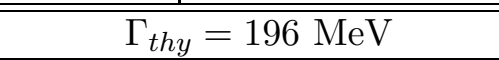 } & \multicolumn{2}{|c|}{$\bar{\Gamma}_{t h y}=200 \mathrm{MeV}$} \\
\hline & \multicolumn{2}{|c|}{$\Gamma_{\text {expt }}=232 \pm 21 \mathrm{MeV}$} & \multicolumn{2}{|c|}{$\Gamma_{\text {expt }}=250-260 \mathrm{MeV}$} \\
\hline
\end{tabular}

\begin{tabular}{||c||c|c||}
\hline \multicolumn{3}{||c||}{ Table K3. 2S kaons (alt.) } \\
\hline \multicolumn{1}{||c||}{} & \multicolumn{2}{c||}{$K^{*}(1580)$} \\
\hline \hline$\pi K$ & 61 & ${ }^{1} P_{1}=+0.11$ \\
\hline$\eta K$ & 60 & ${ }^{1} P_{1}=+0.20$ \\
\hline$\eta^{\prime} K$ & 0.5 & ${ }^{1} P_{1}=-0.026$ \\
\hline$\rho K$ & 90 & ${ }^{3} P_{1}=+0.16$ \\
\hline$\omega K$ & 29 & ${ }^{3} P_{1}=+0.16$ \\
\hline$\phi K$ & 8.6 & ${ }^{3} P_{1}=-0.13$ \\
\hline$\pi K^{*}$ & 99 & ${ }^{3} P_{1}=-0.17$ \\
\hline$\eta K^{*}$ & 1.1 & ${ }^{3} P_{1}=+0.039$ \\
\hline$\pi K_{1}(1273)$ & 1.0 & ${ }^{3} S_{1} \equiv 0$ \\
\hline$\pi{ }^{3} D_{1}=+0.030$ \\
\hline$\pi K_{1}(1402)$ & 0.9 & ${ }^{3} S_{1}=-0.054$ \\
\hline$\pi K_{2}^{*}(1429)$ & 0.0 & ${ }^{5} D_{1} \equiv 0$ \\
\hline \hline \multicolumn{2}{|c||}{$D_{1}=-0.0018$} \\
\hline \multicolumn{2}{|c||}{} & \multicolumn{2}{|c||}{$\Gamma_{\text {thy }}=352 \mathrm{MeV}$} \\
\hline
\end{tabular}




\begin{tabular}{|c|c|c|c|c|}
\hline \multicolumn{5}{|c|}{ Table K4. 3S kaons } \\
\hline & \multicolumn{2}{|r|}{$K^{*}(1950)$} & \multicolumn{2}{|r|}{$K(1830)$} \\
\hline Mode & $\overline{\Gamma_{i}(\mathrm{MeV})}$ & Amps. & $\overline{\Gamma_{i}(\mathrm{MeV})}$ & Amps. \\
\hline 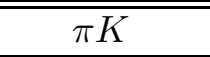 & 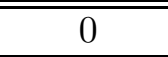 & ${ }^{1} P_{1}=-0.0036$ & - & - \\
\hline$\eta K$ & 1 & ${ }^{1} P_{1}=+0.017$ & - & - \\
\hline$\eta^{\prime} K$ & 0 & ${ }^{1} P_{1}=-0.011$ & - & - \\
\hline$\rho K$ & 8 & ${ }^{3} P_{1}=+0.034$ & 21 & ${ }^{3} P_{0}=-0.061$ \\
\hline$\omega K$ & 3 & ${ }^{3} P_{1}=+0.035$ & 7 & ${ }^{3} P_{0}=+0.062$ \\
\hline$\phi K$ & 12 & ${ }^{3} P_{1}=-0.083$ & 18 & ${ }^{3} P_{0}=-0.12$ \\
\hline$\pi K^{*}$ & 5 & ${ }^{3} P_{1}=-0.028$ & 16 & ${ }^{3} P_{0}=-0.053$ \\
\hline$\eta K^{*}$ & 0 & ${ }^{3} P_{1}=+0.015$ & 27 & ${ }^{3} P_{0}=-0.13$ \\
\hline$\eta^{\prime} K^{*}$ & 10 & ${ }^{3} P_{1}=-0.11$ & - & - \\
\hline$\rho K^{*}$ & 73 & $\begin{array}{l}{ }^{1} P_{1}=+0.027 \\
{ }^{3} P_{1} \equiv 0 \\
{ }^{5} P_{1}=-0.12 \\
{ }^{5} F_{1} \equiv 0\end{array}$ & 45 & ${ }^{3} P_{0}=-0.12$ \\
\hline$\omega K^{*}$ & 24 & $\begin{array}{l}{ }^{1} P_{1}=+0.028 \\
{ }^{3} P_{1} \equiv 0 \\
{ }^{5} P_{1}=-0.12 \\
{ }^{5} F_{1} \equiv 0\end{array}$ & 14 & ${ }^{3} P_{0}=-0.12$ \\
\hline$b_{1} K$ & 10 & $\begin{array}{l}{ }^{3} S_{1}=+0.024 \\
{ }^{3} D_{1}=+0.049\end{array}$ & - & - \\
\hline$h_{1} K$ & 2 & $\begin{array}{l}{ }^{3} S_{1}=+0.033 \\
{ }^{3} D_{1}=+0.037\end{array}$ & - & - \\
\hline$a_{1} K$ & 8 & $\begin{array}{l}{ }^{3} S_{1}=+0.034 \\
{ }^{3} D_{1}=-0.035\end{array}$ & - & - \\
\hline$f_{1} K$ & 3 & $\begin{array}{l}{ }^{3} S_{1}=+0.044 \\
{ }^{3} D_{1}=-0.028\end{array}$ & - & - \\
\hline$a_{2} K$ & 4 & ${ }^{5} D_{1}=+0.040$ & 0 & ${ }^{5} D_{0}=-0.0071$ \\
\hline$f_{2} K$ & 3 & ${ }^{5} D_{1}=+0.050$ & 0 & ${ }^{5} D_{0}=-0.025$ \\
\hline$\pi(1300) K$ & 18 & ${ }^{1} P_{1}=+0.082$ & - & - \\
\hline$\eta(1293) K$ & 6 & ${ }^{1} P_{1}=+0.083$ & - & - \\
\hline$\omega(1419) K$ & 2 & ${ }^{1} P_{1}=+0.067$ & - & - \\
\hline$h_{1}(1386) K$ & 3 & $\begin{array}{l}{ }^{3} S_{1}=-0.069 \\
{ }^{3} D_{1}=-0.024\end{array}$ & - & - \\
\hline$f_{1}^{s \bar{s}}(1426) K$ & 4 & $\begin{array}{l}{ }^{3} S_{1}=+0.11 \\
{ }^{3} D_{1}=-0.0073\end{array}$ & - & - \\
\hline$\eta_{s}(1415) K$ & 2 & ${ }^{1} P_{1}=+0.070$ & - & - \\
\hline$\pi K_{1}(1273)$ & 30 & $\begin{array}{l}{ }^{3} S_{1} \equiv 0 \\
{ }^{3} D_{1}=+0.085\end{array}$ & - & - \\
\hline$\pi K_{0}^{*}(1412)$ & - & - & 7 & ${ }^{1} S_{0}=-0.059$ \\
\hline$\eta K_{1}(1273)$ & 4 & $\begin{array}{l}{ }^{3} S_{1} \equiv 0 \\
{ }^{3} D_{1}=+0.040\end{array}$ & - & - \\
\hline$\pi K_{1}(1402)$ & 3 & $\begin{array}{l}{ }^{3} S_{1}=-0.032 \\
{ }^{3} D_{1} \equiv 0\end{array}$ & - & - \\
\hline$\pi K_{2}^{*}(1429)$ & 11 & ${ }^{5} D_{1}=-0.063$ & 6 & ${ }^{5} D_{0}=-0.059$ \\
\hline
\end{tabular}




\begin{tabular}{|c|c|c|c|c|}
\hline \multicolumn{5}{|c|}{ Table K4. 3S kaons (cont.) } \\
\hline & \multicolumn{2}{|r|}{$K^{*}(1950)$} & \multicolumn{2}{|c|}{$K(1830)$} \\
\hline Mode & $\Gamma_{i}(\mathrm{MeV})$ & Amps. & $\Gamma_{i}(\mathrm{MeV})$ & Amps. \\
\hline$\overline{\bar{\pi} \pi K^{*}(1414)}$ & 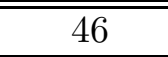 & ${ }^{3} P_{1}=-0.12$ & 40 & ${ }^{3} P_{0}=-0.14$ \\
\hline$\pi K(1460)$ & 20 & ${ }^{1} P_{1}=+0.087$ & - & - \\
\hline$\pi K^{*}(1717)$ & 0 & ${ }^{3} P_{1}=+0.0012$ & - & - \\
\hline$\pi K_{2}(1773)$ & 0 & $\begin{array}{l}{ }^{5} P_{1}=+0.0013 c_{D}-0.0016 s_{D} \\
{ }^{5} F_{1}=+0.00035 c_{D}+0.00029 s_{D}\end{array}$ & - & - \\
\hline \multirow[t]{3}{*}{$\pi K_{3}^{*}(1776)$} & 0 & ${ }^{7} F_{1}=-0.00036$ & - & - \\
\hline & & $\Gamma_{t h y}=320 \mathrm{MeV}$ & \multicolumn{2}{|c|}{$\overline{\Gamma_{\text {thy }}=201 \mathrm{MeV}}$} \\
\hline & & & \multicolumn{2}{|c|}{$\Gamma_{\text {expt }} \sim 250 \mathrm{MeV}$} \\
\hline
\end{tabular}

\begin{tabular}{|c|c|c|c|c|}
\hline \multicolumn{5}{|c|}{ Table K5. $1^{3} \mathrm{P}_{\mathrm{J}}$ kaons } \\
\hline & \multicolumn{2}{|c|}{$K_{2}^{*}(1429)$} & \multicolumn{2}{|c|}{$K_{0}^{*}(1412)$} \\
\hline Mode & $\Gamma_{i}(\mathrm{MeV})$ & Amps. & $\Gamma_{i}(\mathrm{MeV})$ & Amps. \\
\hline$\overline{\pi K}$ & 56 & ${ }^{1} D_{2}=+0.12$ & $\overline{113}$ & ${ }^{1} S_{0}=+0.17$ \\
\hline$\eta K$ & 1 & ${ }^{1} D_{2}=-0.023$ & 6 & ${ }^{1} S_{0}=-0.075$ \\
\hline$\rho K$ & 4 & ${ }^{3} D_{2}=+0.045$ & - & - \\
\hline$\omega K$ & 1 & ${ }^{3} D_{2}=+0.042$ & - & - \\
\hline$\pi K^{*}$ & 13 & ${ }^{3} P_{1}=-0.073$ & - & - \\
\hline$\pi K_{1}(1273)$ & 0 & $\begin{array}{l}{ }^{3} P_{2}=+0.0093 \\
{ }^{3} F_{2}=+0.000067\end{array}$ & 0 & ${ }^{3} P_{0}=+0.012$ \\
\hline & \multicolumn{2}{|c|}{ 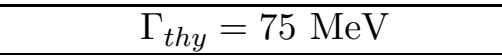 } & \multicolumn{2}{|c|}{$\bar{\Gamma}_{t h y}=119 \mathrm{MeV}$} \\
\hline & \multicolumn{2}{|c|}{$\Gamma_{\text {expt }}=99 \pm 3 \mathrm{MeV}$} & \multicolumn{2}{|c|}{$\Gamma_{\text {expt }}=294 \pm 23 \mathrm{MeV}$} \\
\hline
\end{tabular}

\begin{tabular}{|c|c|c|}
\hline \multicolumn{3}{|c|}{ Table K6. $1^{2 S+1} \mathrm{P}_{1}$ kaons, general mixing } \\
\hline & \multicolumn{2}{|c|}{$K_{1}(1273)=c\left|1^{1} \mathrm{P}_{1}\right\rangle+s\left|1^{3} \mathrm{P}_{1}\right\rangle$} \\
\hline Mode & $\Gamma_{i}(\mathrm{MeV})$ & Amps. \\
\hline$\rho K$ & $21.8 c^{2}+61.6 s c+43.6 s^{2}$ & $\begin{array}{l}{ }^{3} S_{1}=-0.242 c-0.342 s \\
{ }^{3} D_{1}=-0.00202 c+0.00143 s\end{array}$ \\
\hline$\pi K^{*}$ & $59.6 c^{2}-158.7 s c+115.7 s^{2}$ & $\begin{array}{l}{ }^{3} S_{1}-0.204 c+0.288 s \\
{ }^{3} D_{1}=-0.0411 c-0.290 s\end{array}$ \\
\hline & \multicolumn{2}{|c|}{ 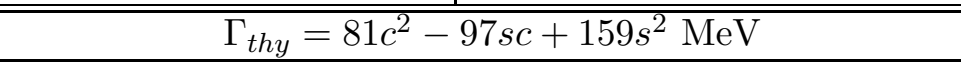 } \\
\hline & \multicolumn{2}{|c|}{$\Gamma_{\text {expt }}=90 \pm 20 \mathrm{MeV}$} \\
\hline
\end{tabular}




\begin{tabular}{||c||c|c||}
\hline \multicolumn{3}{||c||}{ Table K7. $1^{2 S+1} \mathrm{P}_{1}$ kaons, general mixing } \\
\hline Mode & $K_{1}(1402)=-s\left|1^{1} \mathrm{P}_{1}\right\rangle+c\left|1^{3} \mathrm{P}_{1}\right\rangle$ \\
\hline \hline$\rho K$ & $160.0 c^{2}-219.9 s c+82.3 s^{2}$ & $\begin{array}{l}{ }^{3} S_{1}=-0.290 c+0.205 s \\
3\end{array} D_{1}=+0.0282 c+0.0399 s$ \\
\hline$\omega K$ & $52.3 c^{2}-72.3 s c+26.8 s^{2}$ & $\begin{array}{l}3 \\
{ }^{3} S_{1}=-0.294 c+0.208 s \\
{ }^{3} D_{1}=+0.0259 c+0.0366 s\end{array}$ \\
\hline$\pi K^{*}$ & $141.1 c^{2}+176.2 s c+78.8 s^{2}$ & $\begin{array}{l}{ }^{3} S_{1}=+0.247 c+0.175 s \\
{ }^{3} D_{1}=-0.0498 c+0.0704 s\end{array}$ \\
\hline \hline \multicolumn{2}{||c||}{$\Gamma_{t h y}=353 c^{2}-116 s c+188 s^{2} \mathrm{MeV}$} \\
\hline
\end{tabular}

\begin{tabular}{|c|c|c|c|c|}
\hline \multicolumn{5}{|c|}{ Table K8. ${ }^{2 S+1} \mathrm{P}_{1}$ kaons, HQET magic mixing } \\
\hline & \multicolumn{2}{|r|}{$K_{1}(1273)$} & \multicolumn{2}{|c|}{$K_{1}(1402)$} \\
\hline Mode & $\Gamma_{i}(\mathrm{MeV})$ & Amps. & $\overline{\Gamma_{i}(\mathrm{MeV})}$ & Amps. \\
\hline$\rho K$ & 58 & $\begin{array}{l}{ }^{3} S_{1}=-0.39 \\
{ }^{3} D_{1}=-0.00083\end{array}$ & 30 & $\begin{array}{l}{ }^{3} S_{1}=-0.12 \\
{ }^{3} D_{1}=+0.046\end{array}$ \\
\hline$\omega K$ & - & - & 10 & $\begin{array}{l}{ }^{3} S_{1}=-0.12 \\
{ }^{3} D_{1}=+0.042\end{array}$ \\
\hline$\pi K^{*}$ & 3 & $\begin{array}{l}{ }^{3} S_{1} \equiv 0 \\
{ }^{3} D_{1}=-0.050\end{array}$ & 203 & $\begin{array}{l}{ }^{3} S_{1}=+0.30 \\
{ }^{3} D_{1} \equiv 0\end{array}$ \\
\hline & \multicolumn{2}{|c|}{ 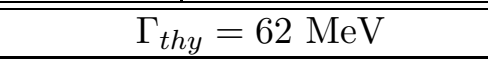 } & \multicolumn{2}{|c|}{$\bar{\Gamma}_{\text {thy }}=244 \mathrm{MeV}$} \\
\hline & \multicolumn{2}{|c|}{$\Gamma_{\text {expt }}=90 \pm 20 \mathrm{MeV}$} & \multicolumn{2}{|c|}{$\Gamma_{\text {expt }}=174 \pm 13 \mathrm{MeV}$} \\
\hline
\end{tabular}




\begin{tabular}{|c|c|c|c|c|}
\hline \multicolumn{5}{|c|}{ Table K9. $2^{3} \mathrm{P}_{\mathrm{J}}$ kaons } \\
\hline & \multicolumn{2}{|c|}{$K_{2}^{*}(1850)$} & \multicolumn{2}{|c|}{$K_{0}^{*}(1850)$} \\
\hline Mode & $\overline{\Gamma_{i}(\mathrm{MeV})}$ & Amps. & $\overline{\Gamma_{i}(\mathrm{MeV})}$ & Amps. \\
\hline 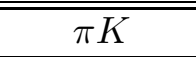 & 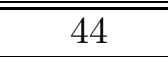 & 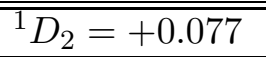 & 29 & ${ }^{1}{ }^{1} S_{0}=-0.062$ \\
\hline$\eta K$ & 1 & ${ }^{1} D_{2}=-0.023$ & 0 & ${ }^{1} S_{0}=+0.011$ \\
\hline$\eta^{\prime} K$ & 15 & ${ }^{1} D_{2}=+0.10$ & 11 & ${ }^{1} S_{0}=+0.083$ \\
\hline$\rho K$ & 44 & ${ }^{3} D_{2}=+0.087$ & - & - \\
\hline$\omega K$ & 14 & ${ }^{3} D_{2}=+0.086$ & - & - \\
\hline$\phi K$ & 12 & ${ }^{3} D_{2}=+0.091$ & - & - \\
\hline$\pi K^{*}$ & 47 & ${ }^{3} D_{2}=-0.090$ & - & - \\
\hline$\eta K^{*}$ & 26 & ${ }^{3} D_{2}=-0.13$ & - & - \\
\hline$\rho K^{*}$ & 78 & $\begin{array}{l}{ }^{5} S_{2}=-0.14 \\
{ }^{1} D_{2}=+0.021 \\
{ }^{3} D_{2} \equiv 0 \\
{ }^{5} D_{2}=-0.056 \\
{ }^{5} G_{2} \equiv 0\end{array}$ & 48 & $\begin{array}{l}{ }^{1} S_{0}=+0.068 \\
{ }^{5} D_{0}=-0.095\end{array}$ \\
\hline$\omega K^{*}$ & 27 & $\begin{array}{l}{ }^{5} S_{2}=-0.14 \\
{ }^{1} D_{2}=+0.020 \\
{ }^{3} D_{2} \equiv 0 \\
{ }^{5} D_{2}=-0.054 \\
{ }^{5} G_{2} \equiv 0\end{array}$ & 15 & $\begin{array}{l}{ }^{1} S_{0}=+0.071 \\
{ }^{5} D_{0}=-0.091\end{array}$ \\
\hline$b_{1} K$ & 8 & $\begin{array}{l}{ }^{3} P_{2}=+0.058 \\
{ }^{3} F_{2}=+0.0071\end{array}$ & 49 & ${ }^{3} P_{0}=+0.15$ \\
\hline$h_{1} K$ & 4 & $\begin{array}{l}{ }^{3} P_{2}=+0.065 \\
{ }^{3} F_{2}=+0.013\end{array}$ & 26 & ${ }^{3} P_{0}=+0.16$ \\
\hline$a_{1} K$ & 3 & $\begin{array}{l}{ }^{3} P_{2}=+0.037 \\
{ }^{3} F_{2}=-0.0050\end{array}$ & 23 & ${ }^{3} P_{0}=-0.10$ \\
\hline$f_{1} K$ & 1 & $\begin{array}{l}{ }^{3} P_{2}=+0.031 \\
{ }^{3} F_{2}=-0.0022\end{array}$ & 4 & ${ }^{3} P_{0}=-0.080$ \\
\hline$a_{2} K$ & 3 & $\begin{array}{l}{ }^{5} P_{2}=+0.047 \\
{ }^{5} F_{2}=+0.0011\end{array}$ & - & - \\
\hline$f_{2} K$ & 3 & $\begin{array}{l}{ }^{5} P_{2}=+0.066 \\
{ }^{5} F_{2}=+0.0036\end{array}$ & - & - \\
\hline$\pi(1300) K$ & 0 & ${ }^{1} D_{2}=+0.013$ & 48 & ${ }^{1} S_{0}=+0.18$ \\
\hline$\eta(1293) K$ & 0 & ${ }^{1} D_{2}=+0.015$ & 16 & ${ }^{1} S_{0}=+0.18$ \\
\hline$\pi K_{1}(1273)$ & 6 & $\begin{array}{l}{ }^{3} P_{2}=+0.033 \\
{ }^{3} F_{2}=+0.029\end{array}$ & 149 & ${ }^{3} P_{0}=+0.22$ \\
\hline$\eta K_{1}(1273)$ & 1 & $\begin{array}{l}{ }^{3} P_{2}=+0.050 \\
{ }^{3} F_{2}=+0.0012\end{array}$ & 4 & ${ }^{3} P_{0}=+0.11$ \\
\hline$\pi K_{1}(1402)$ & 11 & $\begin{array}{l}{ }^{3} P_{2}=-0.071 \\
{ }^{3} F_{2} \equiv 0\end{array}$ & 0 & ${ }^{3} P_{0}=-0.0031$ \\
\hline$\pi K_{2}^{*}(1429)$ & 15 & $\begin{array}{l}{ }^{5} P_{2}=-0.086 \\
{ }^{5} F_{2}=-0.011\end{array}$ & - & - \\
\hline$\pi K^{*}(1414)$ & 5 & ${ }^{3} D_{2}=-0.049$ & - & - \\
\hline$\pi K(1460)$ & 2 & ${ }^{1} D_{2}=+0.033$ & 33 & ${ }^{1} S_{0}=+0.14$ \\
\hline & $\overline{\overline{\Gamma_{t h}}}$ & $=370 \mathrm{MeV}$ & $\overline{\overline{\Gamma_{t h}}}$ & $=455 \mathrm{MeV}$ \\
\hline
\end{tabular}




\begin{tabular}{|c|c|c|}
\hline \multicolumn{3}{|c|}{ Table K10. $2^{2 S+1} \mathrm{P}_{1}$ kaons, general mixing (two states) } \\
\hline & \multicolumn{2}{|c|}{$K_{1}^{a}(1800)=c\left|2^{1} \mathrm{P}_{1}\right\rangle+s\left|2^{3} \mathrm{P}_{1}\right\rangle$} \\
\hline Mode & $\Gamma_{i}(\mathrm{MeV})$ & Amps. \\
\hline$\rho K$ & $40.8 c^{2}-56.4 s c+20.9 s^{2}$ & $\begin{array}{l}{ }^{3} S_{1}=-0.00776 c-0.0110 s \\
{ }^{3} D_{1}=-0.0874 c+0.0618 s\end{array}$ \\
\hline$\omega K$ & $13.2 c^{2}-18.1 s c+6.8 s^{2}$ & $\begin{array}{l}{ }^{3} S_{1}=-0.00941 c-0.0133 s \\
{ }^{3} D_{1}=-0.0865 c+0.0612 s\end{array}$ \\
\hline$\phi K$ & $15.4 c^{2}-6.6 s c+17.8 s^{2}$ & $\begin{array}{l}{ }^{3} S_{1}=+0.0736 c-0.104 s \\
{ }^{3} D_{1}=+0.0841 c+0.0595 s\end{array}$ \\
\hline$\pi K^{*}$ & $45.2 c^{2}+63.9 s c+22.6 s^{2}$ & $\begin{array}{l}{ }^{3} S_{1}=+0.00121 c-0.00171 s \\
{ }^{3} D_{1}=-0.0919 c-0.0650 s\end{array}$ \\
\hline$\eta K^{*}$ & $0.8 c^{2}-2.8 s c+21.1 s^{2}$ & $\begin{array}{l}{ }^{3} S_{1}=+0.0102 c+0.0839 s \\
{ }^{3} D_{1}=+0.0209 c-0.0860 s\end{array}$ \\
\hline$\rho K^{*}$ & $47.9 c^{2}+11.4 s^{2}$ & $\begin{array}{l}{ }^{3} S_{1}=-0.118 c \\
{ }^{3} D_{1}=-0.0513 c \\
{ }^{5} D_{1}=-0.0628 s\end{array}$ \\
\hline$\omega K^{*}$ & $16.0 c^{2}+3.1 s^{2}$ & $\begin{array}{l}{ }^{3} S_{1}=-0.123 c \\
{ }^{3} D_{1}=-0.0475 c \\
{ }^{5} D_{1}=-0.0582 s\end{array}$ \\
\hline$b_{1} K$ & $5.4 s^{2}$ & ${ }^{3} P_{1}=-0.0568 s$ \\
\hline$h_{1} K$ & $4.1 s^{2}$ & ${ }^{3} P_{1}=-0.0721 s$ \\
\hline$a_{1} K$ & $1.3 c^{2}-6.2 s c+7.0 s^{2}$ & ${ }^{3} P_{1}=-0.0284 c+0.0647 s$ \\
\hline$f_{1} K$ & $0.1 c^{2}-0.4 s c+0.4 s^{2}$ & ${ }^{3} P_{1}=-0.0162 c+0.0378 s$ \\
\hline$f_{2} K$ & $1.4 c^{2}+2.6 s c+1.2 s^{2}$ & $\begin{array}{l}{ }^{5} P_{1}=-0.0648 c-0.0603 s \\
{ }^{5} F_{1}=-0.00104 c+0.000733 s\end{array}$ \\
\hline$\pi K_{1}(1273)$ & $1.8 c^{2}+17.2 s c+42.3 s^{2}$ & ${ }^{3} P_{1}=-0.0251 c-0.123 s$ \\
\hline$\pi K_{1}(1402)$ & $1.7 c^{2}+2.6 s c+1.0 s^{2}$ & ${ }^{3} P_{1}=-0.0305 c-0.0240 s$ \\
\hline$\pi K_{0}^{*}(1412)$ & $3.5 c^{2}+4.6 s c+1.5 s^{2}$ & ${ }^{1} P_{1}=-0.0450 c-0.0299 s$ \\
\hline$\pi K_{2}^{*}(1429)$ & $23.8 c^{2}-43.6 s c+20.4 s^{2}$ & $\begin{array}{l}{ }^{5} P_{1}=-0.122 c+0.113 s \\
{ }^{5} F_{1}=-0.00941 c-0.00665 s\end{array}$ \\
\hline$\pi K^{*}(1414)$ & $14.1 c^{2}-27.2 s c+23.7 s^{2}$ & $\begin{array}{l}{ }^{3} S_{1}=-0.0806 c+0.114 s \\
{ }^{3} D_{1}=-0.0416 c-0.0294 s\end{array}$ \\
\hline & $\Gamma_{t h y}=227$ & $70 s c+212 s^{2} \mathrm{MeV}$ \\
\hline
\end{tabular}




\begin{tabular}{|c|c|c|c|c|}
\hline \multicolumn{5}{|c|}{ Table K11. $1^{3} \mathrm{D}_{\mathrm{J}}$ kaons } \\
\hline & \multicolumn{2}{|r|}{$K_{3}^{*}(1776)$} & \multicolumn{2}{|c|}{$K^{*}(1717)$} \\
\hline Mode & $\overline{\Gamma_{i}(\mathrm{MeV})}$ & Amps. & $\overline{\Gamma_{i}(\mathrm{MeV})}$ & Amps. \\
\hline$\overline{\bar{\pi} \pi K}$ & 40 & 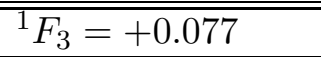 & 45 & ${ }^{1} P_{1}=+0.085$ \\
\hline$\eta \overline{\eta K}$ & 19 & ${ }^{1} F_{3}=+0.098$ & 53 & ${ }^{1} P_{1}=+0.17$ \\
\hline$\eta^{\prime} K$ & 0 & ${ }^{1} F_{3}=-0.0062$ & 1 & ${ }^{1} P_{1}=-0.030$ \\
\hline$\rho K$ & 10 & ${ }^{3} F_{3}=+0.045$ & 26 & ${ }^{3} P_{1}=-0.075$ \\
\hline$\omega K$ & 3 & ${ }^{3} F_{3}=+0.043$ & 8 & ${ }^{3} P_{1}=-0.075$ \\
\hline$\phi K$ & 1 & ${ }^{3} F_{3}=-0.026$ & 9 & ${ }^{3} P_{1}=+0.095$ \\
\hline$\pi K^{*}$ & 14 & ${ }^{3} F_{3}=-0.052$ & 25 & ${ }^{3} P_{1}=+0.074$ \\
\hline$\eta K^{*}$ & 0 & ${ }^{3} F_{3}=+0.0079$ & 1 & ${ }^{3} P_{1}=-0.021$ \\
\hline$\rho K^{*}$ & 42 & $\begin{array}{l}{ }^{5} P_{3}=-0.13 \\
{ }^{1} F_{3}=+0.0033 \\
{ }^{3} F_{3} \equiv 0 \\
{ }^{5} F_{3}=-0.0072 \\
{ }^{5} H_{3} \equiv 0\end{array}$ & 2 & $\begin{array}{l}{ }^{1} P_{1}=+0.035 \\
{ }^{3} P_{1} \equiv 0 \\
{ }^{5} P_{1}=-0.016 \\
{ }^{5} F_{1}=-0.0044\end{array}$ \\
\hline$\omega K^{*}$ & 12 & $\begin{array}{l}{ }^{5} P_{3}=-0.12 \\
{ }^{1} F_{3}=+0.0028 \\
{ }^{3} F_{3} \equiv 0 \\
{ }^{5} F_{3}=-0.0062 \\
{ }^{5} H_{3} \equiv 0\end{array}$ & 1 & $\begin{array}{l}{ }^{1} P_{1}=+0.031 \\
{ }^{3} P_{1} \equiv 0 \\
{ }^{5} P_{1}=-0.014 \\
{ }^{5} F_{1}=-0.0030\end{array}$ \\
\hline$b_{1} K$ & 0 & $\begin{array}{l}{ }^{3} D_{3}=+0.0092 \\
{ }^{3} G_{3}=+0.00012\end{array}$ & - & - \\
\hline$h_{1} K$ & 0 & $\begin{array}{l}{ }^{3} D_{3}=+0.020 \\
{ }^{3} G_{3}=+0.00057\end{array}$ & 33 & $\begin{array}{l}{ }^{3} S_{1}=+0.27 \\
{ }^{3} D_{1}=+0.0040\end{array}$ \\
\hline$a_{1} K$ & 0 & $\begin{array}{l}{ }^{3} D_{3}=+0.0043 \\
{ }^{3} G_{3}=-0.000082\end{array}$ & - & 1 \\
\hline$f_{2} K$ & 0 & $\begin{array}{l}{ }^{5} D_{3}=+0.0012 \\
{ }^{5} G_{3}=+1.1 \cdot 10^{-6}\end{array}$ & - & - \\
\hline$\pi K_{1}(1273)$ & 1 & $\begin{array}{l}{ }^{3} D_{3}=+0.022 \\
{ }^{3} G_{3}=+0.0030\end{array}$ & 145 & $\begin{array}{l}{ }^{3} S_{1}=+0.26 \\
{ }^{3} D_{1}=+0.028\end{array}$ \\
\hline$\pi K_{1}(1402)$ & 1 & $\begin{array}{l}{ }^{3} D_{3}=-0.022 \\
{ }^{3} G_{3} \equiv 0\end{array}$ & 0 & $\begin{array}{l}{ }^{3} S_{1} \equiv 0 \\
{ }^{3} D_{1}=+0.0085\end{array}$ \\
\hline$\pi K_{2}^{*}(1429)$ & 1 & $\begin{array}{l}{ }^{5} D_{3}=-0.027 \\
{ }^{5} G_{3}=-0.00053\end{array}$ & 0 & ${ }^{5} D_{1}=+0.012$ \\
\hline$\pi K^{*}(1414)$ & 0 & ${ }^{3} F_{3}=-0.0049$ & 0 & ${ }^{3} P_{1}=+0.0043$ \\
\hline$\pi K(1460)$ & 0 & ${ }^{1} F_{3}=+0.0027$ & 0 & ${ }^{1} P_{1}=+0.0061$ \\
\hline & \multicolumn{2}{|c|}{$\overline{\Gamma_{t h y}}=145 \mathrm{MeV}$} & \multicolumn{2}{|c|}{$\Gamma_{t h y}=348 \mathrm{MeV}$} \\
\hline & \multicolumn{2}{|c|}{$\Gamma_{\text {expt }}=159 \pm 21 \mathrm{MeV}$} & \multicolumn{2}{|c|}{$\Gamma_{\text {expt }}=322 \pm 110 \mathrm{MeV}$} \\
\hline
\end{tabular}




\begin{tabular}{|c|c|c|}
\hline \multicolumn{3}{|c|}{ Table K12. $1{ }^{2 S+1} \mathrm{D}_{\mathrm{J}}$ kaons, general mixing } \\
\hline & \multicolumn{2}{|c|}{$K_{2}(1773)=c\left|1^{1} \mathrm{D}_{2}\right\rangle+s\left|1^{3} \mathrm{D}_{2}\right\rangle$} \\
\hline Mode & $\Gamma_{i}(\mathrm{MeV})$ & Amps. \\
\hline$\rho K$ & $43.8 c^{2}+64.7 s c+57.0 s^{2}$ & $\begin{array}{l}{ }^{3} P_{2}=-0.0812 c-0.0995 s \\
{ }^{3} F_{2}=-0.0454 c+0.0371 s\end{array}$ \\
\hline$\omega K$ & $14.3 c^{2}+21.8 s c+18.7 s^{2}$ & $\begin{array}{l}{ }^{3} P_{2}=-0.0815 c-0.0998 s \\
{ }^{3} F_{2}=-0.0441 c+0.0360 s\end{array}$ \\
\hline$\phi K$ & $14.8 c^{2}-33.2 s c+21.6 s^{2}$ & $\begin{array}{l}{ }^{3} P_{2}=-0.111 c+0.136 s \\
{ }^{3} F_{2}=-0.0261 c-0.0213 s\end{array}$ \\
\hline$\pi K^{*}$ & $46.5 c^{2}-54.8 s c+57.7 s^{2}$ & $\begin{array}{l}{ }^{3} P_{2}=-0.0792 c+0.0969 s \\
{ }^{3} F_{2}=-0.0532 c-0.0434 s\end{array}$ \\
\hline$\eta K^{*}$ & $29.7 c^{2}+10.4 s c+1.2 s^{2}$ & $\begin{array}{l}{ }^{3} P_{2}=-0.140 c-0.0293 s \\
{ }^{3} F_{2}=-0.0465 c+0.00651 s\end{array}$ \\
\hline$\rho K^{*}$ & $20.3 c^{2}+10.3 s^{2}$ & $\begin{array}{l}{ }^{3} P_{2}=-0.0890 c \\
{ }^{5} P_{2}=-0.0629 s \\
{ }^{3} F_{2}=-0.00918 c \\
{ }^{5} F_{2}=-0.0106 s\end{array}$ \\
\hline$\omega K^{*}$ & $5.9 c^{2}+3.0 s^{2}$ & $\begin{array}{l}{ }^{3} P_{2}=-0.0853 c \\
{ }^{5} P_{2}=-0.0603 s \\
{ }^{3} F_{2}=-0.00780 c \\
{ }^{5} F_{2}=-0.00900 s\end{array}$ \\
\hline$b_{1} K$ & $0.1 s^{2}$ & ${ }^{3} D_{2}=-0.00878 s$ \\
\hline$h_{1} K$ & $0.3 s^{2}$ & ${ }^{3} D_{2}=-0.0198 s$ \\
\hline$a_{1} K$ & $0.1 c^{2}-0.1 s c+0.1 s^{2}$ & ${ }^{3} D_{2}=-0.00760 c+0.00709 s$ \\
\hline$f_{2} K$ & $4.5 c^{2}+10.9 s c+6.7 s^{2}$ & $\begin{array}{l}{ }^{5} S_{2}=-0.229 c-0.281 s \\
{ }^{5} D_{2}=-0.000477 c-0.000117 s \\
{ }^{5} G_{2}=-2.18 \cdot 10^{-7} c+1.78 \cdot 10^{-7} s\end{array}$ \\
\hline$\pi K_{1}(1273)$ & $1.1 c^{2}+5.3 s c+6.6 s^{2}$ & ${ }^{3} D_{2}=-0.0204 c-0.0508 s$ \\
\hline$\pi K_{1}(1402)$ & $0.4 c^{2}+0.1 s c$ & ${ }^{3} D_{2}=-0.0168 c-0.00138 s$ \\
\hline$\pi K_{0}^{*}(1412)$ & $0.1 c^{2}+0.4 s c+0.4 s^{2}$ & ${ }^{1} D_{2}=-0.00783 c-0.0159 s$ \\
\hline$\pi K_{2}^{*}(1429)$ & $51.1 c^{2}-123.3 s c+75.3 s^{2}$ & $\begin{array}{l}{ }^{5} S_{2}=-0.190 c+0.233 s \\
{ }^{5} D_{2}=-0.0254 c+0.00603 s \\
{ }^{5} G_{2}=-0.000668 c-0.000545 s\end{array}$ \\
\hline$\pi K^{*}(1414)$ & $0.1 c^{2}+0.1 s^{2}$ & $\begin{array}{l}{ }^{3} P_{2}=-0.00428 c+0.00524 s \\
{ }^{3} F_{2}=-0.00484 c-0.00396 s\end{array}$ \\
\hline & $\overline{\Gamma_{t h y}}=233$ & $-98 s c+259 s^{2} \mathrm{MeV}$ \\
\hline & $\Gamma_{\text {expt }}$ & $186 \pm 14 \mathrm{MeV}$ \\
\hline
\end{tabular}




\begin{tabular}{|c|c|c|}
\hline \multicolumn{3}{|c|}{ Table K13. $1^{2 S+1} \mathrm{D}_{\mathrm{J}}$ kaons, general mixing } \\
\hline & \multicolumn{2}{|c|}{$K_{2}(1816)=-s\left|1^{1} \mathrm{D}_{2}\right\rangle+c\left|1^{3} \mathrm{D}_{2}\right\rangle$} \\
\hline Mode & $\Gamma_{i}(\mathrm{MeV})$ & Amps. \\
\hline$\rho K$ & $61.5 c^{2}-61.2 s c+49.0 s^{2}$ & $\begin{array}{l}{ }^{3} P_{2}=-0.0976 c+0.0797 s \\
{ }^{3} F_{2}=+0.0420 c+0.0514 s\end{array}$ \\
\hline$\omega K$ & $20.3 c^{2}-20.8 s c+16.0 s^{2}$ & $\begin{array}{l}{ }^{3} P_{2}=-0.0981 c+0.0801 s \\
{ }^{3} F_{2}=+0.0409 c+0.0501 s\end{array}$ \\
\hline$\phi K$ & $26.1 c^{2}+38.8 s c+18.2 s^{2}$ & $\begin{array}{l}{ }^{3} P_{2}=+0.140 c+0.114 s \\
{ }^{3} F_{2}=-0.0270 c+0.0331 s\end{array}$ \\
\hline$\pi K^{*}$ & $61.7 c^{2}+48.7 s c+51.8 s^{2}$ & $\begin{array}{l}{ }^{3} P_{2}=+0.0945 c+0.0771 s \\
{ }^{3} F_{2}=-0.0482 c+0.0590 s\end{array}$ \\
\hline$\eta K^{*}$ & $1.4 c^{2}-11.2 s c+34.6 s^{2}$ & $\begin{array}{l}{ }^{3} P_{2}=-0.0296 c+0.141 s \\
{ }^{3} F_{2}=+0.00784 c+0.0560 s\end{array}$ \\
\hline$\rho K^{*}$ & $16.4 c^{2}+31.7 s^{2}$ & $\begin{array}{l}{ }^{3} P_{2}=+0.100 s \\
{ }^{5} P_{2}=-0.0708 c \\
{ }^{3} F_{2}=+0.0151 s \\
{ }^{5} F_{2}=-0.0174 c\end{array}$ \\
\hline$\omega K^{*}$ & $5.0 c^{2}+9.6 s^{2}$ & $\begin{array}{l}{ }^{3} P_{2}=+0.0976 s \\
{ }^{5} P_{2}=-0.0690 c \\
{ }^{3} F_{2}=+0.0135 s \\
{ }^{5} F_{2}=-0.0156 c\end{array}$ \\
\hline$h_{1} K$ & $0.7 c^{2}$ & ${ }^{3} D_{2}=-0.0278 c$ \\
\hline$b_{1} K$ & $0.5 c^{2}$ & ${ }^{3} D_{2}=-0.0169 c$ \\
\hline$a_{1} K$ & $0.3 c^{2}+0.7 s c+0.4 s^{2}$ & ${ }^{3} D_{2}=+0.0134 c+0.0146 s$ \\
\hline$f_{1} K$ & 0.0 & ${ }^{3} D_{2}=+0.00582 c+0.00621 s$ \\
\hline$a_{2} K$ & $20.4 c^{2}-33.3 s c+13.6 s^{2}$ & $\begin{array}{l}{ }^{5} S_{2}=-0.281 c+0.229 s \\
{ }^{5} D_{2}=-0.000118 c+0.000481 s \\
{ }^{5} G_{2}=+1.81 \cdot 10^{-7} c+2.22 \cdot 10^{-7} s\end{array}$ \\
\hline$f_{2} K$ & $29.1 c^{2}-47.5 s c+19.4 s^{2}$ & $\begin{array}{l}{ }^{5} S_{2}=-0.261 c+0.213 s \\
{ }^{5} D_{2}=-0.00260 c+0.0107 s \\
{ }^{5} G_{2}=+0.0000928 c+0.000114 s\end{array}$ \\
\hline$\pi K_{1}(1273)$ & $9.9 c^{2}-8.0 s c+1.6 s^{2}$ & ${ }^{3} D_{2}=-0.0582 c+0.0235 s$ \\
\hline$\pi K_{1}(1402)$ & $-0.1 s c+0.8 s^{2}$ & ${ }^{3} D_{2}=-0.00142 c+0.0208 s$ \\
\hline$\pi K_{0}^{*}(1412)$ & $0.7 c^{2}-0.7 s c+0.2 s^{2}$ & ${ }^{1} D_{2}=-0.0199 c+0.00954 s$ \\
\hline$\pi K_{2}^{*}(1429)$ & $82.9 c^{2}+136.1 s c+57.0 s^{2}$ & $\begin{array}{l}{ }^{5} S_{2}=+0.219 c+0.197 s \\
{ }^{5} D_{2}=+0.00757 c+0.0321 s \\
{ }^{5} G_{2}=-0.000895 c+0.00110 s\end{array}$ \\
\hline$\pi K^{*}(1414)$ & $0.1 c^{2}-0.1 s c+0.1 s^{2}$ & $\begin{array}{l}{ }^{3} P_{2}=+0.00410 c+0.00334 s \\
{ }^{3} F_{2}=-0.00557 c+0.00682 s\end{array}$ \\
\hline & $\Gamma_{\text {thy }}=337$ & $+4+41 s c+304 s^{2} \mathrm{MeV}$ \\
\hline & $\overline{\Gamma_{e x}}$ & $276 \pm 35 \mathrm{MeV}$ \\
\hline
\end{tabular}




\begin{tabular}{|c|c|c|c|c|}
\hline \multicolumn{5}{|c|}{ Table K14. $1^{3} \mathrm{~F}_{\mathrm{J}}$ kaons } \\
\hline & \multicolumn{2}{|r|}{$K_{4}^{*}(2045)$} & \multicolumn{2}{|c|}{$K_{2}^{*}(2050)$} \\
\hline Mode & $\overline{\Gamma_{i}(\mathrm{MeV})}$ & Amps. & $\Gamma_{i}(\mathrm{MeV})$ & Amps. \\
\hline 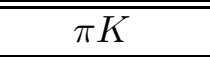 & 21 & ${ }^{1} G_{4}=+0.048$ & 20 & 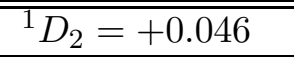 \\
\hline$\eta K$ & 0 & ${ }^{1} G_{4}=-0.011$ & 1 & ${ }^{1} D_{2}=-0.015$ \\
\hline$\eta^{\prime} K$ & 2 & ${ }^{1} G_{4}=+0.029$ & 15 & ${ }^{1} D_{2}=+0.083$ \\
\hline$\rho K$ & 7 & ${ }^{3} G_{4}=+0.030$ & 13 & ${ }^{3} D_{2}=-0.042$ \\
\hline$\omega K$ & 2 & ${ }^{3} G_{4}=+0.029$ & 4 & ${ }^{3} D_{2}=-0.041$ \\
\hline$\phi K$ & 1 & ${ }^{3} G_{4}=+0.022$ & 6 & ${ }^{3} D_{2}=-0.054$ \\
\hline$\pi K^{*}$ & 8 & ${ }^{3} G_{4}=-0.033$ & 13 & ${ }^{3} D_{2}=+0.042$ \\
\hline$\eta K^{*}$ & 3 & ${ }^{3} G_{4}=-0.035$ & 11 & ${ }^{3} D_{2}=+0.068$ \\
\hline$\eta^{\prime} K^{*}$ & 0 & ${ }^{3} G_{4}=+0.00096$ & 0 & ${ }^{3} D_{2}=-0.0066$ \\
\hline$\rho K^{*}$ & 29 & $\begin{array}{l}{ }^{5} D_{4}=-0.070 \\
{ }^{1} G_{4}=+0.0055 \\
{ }^{3} G_{4} \equiv 0 \\
{ }^{5} G_{4}=-0.011 \\
{ }^{5} I_{4} \equiv 0\end{array}$ & 8 & $\begin{array}{l}{ }^{5} S_{2} \equiv 0 \\
{ }^{1} D_{2}=+0.024 \\
{ }^{3} D_{2} \equiv 0 \\
{ }^{5} D_{2}=-0.018 \\
{ }^{5} G_{2}=-0.022\end{array}$ \\
\hline$\omega K^{*}$ & 9 & $\begin{array}{l}{ }^{5} D_{4}=-0.069 \\
{ }^{1} G_{4}=+0.0052 \\
{ }^{3} G_{4} \equiv 0 \\
{ }^{5} G_{4}=-0.010 \\
{ }^{5} I_{4} \equiv 0\end{array}$ & 3 & $\begin{array}{l}{ }^{5} S_{2} \equiv 0 \\
{ }^{1} D_{2}=+0.024 \\
{ }^{3} D_{2} \equiv 0 \\
{ }^{5} D_{2}=-0.018 \\
{ }^{5} G_{2}=-0.021\end{array}$ \\
\hline$\phi K^{*}$ & 3 & $\begin{array}{l}{ }^{5} D_{4}=+0.048 \\
{ }^{1} G_{4}=-0.0012 \\
{ }^{3} G_{4} \equiv 0 \\
{ }^{5} G_{4}=+0.0024 \\
{ }^{5} I_{4} \equiv 0\end{array}$ & 1 & $\begin{array}{l}{ }^{5} S_{2} \equiv 0 \\
{ }^{1} D_{2}=-0.017 \\
{ }^{3} D_{2} \equiv 0 \\
{ }^{5} D_{2}=+0.013 \\
{ }^{5} G_{2}=+0.0050\end{array}$ \\
\hline$b_{1} K$ & 2 & $\begin{array}{l}{ }^{3} F_{4}=+0.022 \\
{ }^{3} H_{4}=+0.0012\end{array}$ & 50 & $\begin{array}{l}{ }^{3} P_{2}=+0.11 \\
{ }^{3} F_{2}=+0.0054\end{array}$ \\
\hline$h_{1} K$ & 1 & $\begin{array}{l}{ }^{3} F_{4}=+0.027 \\
{ }^{3} H_{4}=+0.0019\end{array}$ & 18 & $\begin{array}{l}{ }^{3} P_{2}=+0.10 \\
{ }^{3} F_{2}=+0.0065\end{array}$ \\
\hline$h_{1}(1386) K$ & 0 & $\begin{array}{l}{ }^{3} F_{4}=+0.012 \\
{ }^{3} H_{4}=+0.00032\end{array}$ & 18 & $\begin{array}{l}{ }^{3} P_{2}=+0.13 \\
{ }^{3} F_{2}=+0.0034\end{array}$ \\
\hline$a_{1} K$ & 1 & $\begin{array}{l}{ }^{3} F_{4}=+0.012 \\
{ }^{3} H_{4}=-0.00087\end{array}$ & 26 & $\begin{array}{l}{ }^{3} P_{2}=-0.074 \\
{ }^{3} F_{2}=-0.011\end{array}$ \\
\hline$f_{1} K$ & 0 & $\begin{array}{l}{ }^{3} F_{4}=+0.0093 \\
{ }^{3} H_{4}=-0.00056\end{array}$ & 7 & $\begin{array}{l}{ }^{3} P_{2}=-0.074 \\
{ }^{3} F_{2}=-0.0082\end{array}$ \\
\hline$f_{1}^{s \bar{s}}(1426) K$ & 0 & $\begin{array}{l}{ }^{3} F_{4}=-0.0043 \\
{ }^{3} H_{4}=+0.000011\end{array}$ & 6 & $\begin{array}{l}{ }^{3} P_{2}=+0.087 \\
{ }^{3} F_{2}=+0.0037\end{array}$ \\
\hline$a_{2} K$ & 1 & $\begin{array}{l}{ }^{5} F_{4}=+0.015 \\
{ }^{5} H_{4}=+0.00048\end{array}$ & 7 & $\begin{array}{l}{ }^{5} P_{2}=-0.042 \\
{ }^{5} F_{2}=-0.0084\end{array}$ \\
\hline$f_{2} K$ & 1 & $\begin{array}{l}{ }^{5} F_{4}=+0.020 \\
{ }^{5} H_{4}=+0.00073\end{array}$ & 3 & $\begin{array}{l}{ }^{5} P_{2}=-0.043 \\
{ }^{5} F_{2}=-0.011\end{array}$ \\
\hline$f_{2}^{\prime}(1525) K$ & 0 & $\begin{array}{l}{ }^{5} F_{4}=-0.00073 \\
{ }^{5} H_{4}=-2.2 \cdot 10^{-6}\end{array}$ & 0 & $\begin{array}{l}{ }^{5} P_{2}=+0.027 \\
{ }^{5} F_{2}=+0.00053\end{array}$ \\
\hline
\end{tabular}




\begin{tabular}{|c|c|c|c|c|}
\hline \multicolumn{5}{|c|}{ Table K14. $1^{3} \mathrm{~F}_{\mathrm{J}}$ kaons (cont.) } \\
\hline & \multicolumn{2}{|r|}{$K_{4}^{*}(2045)$} & \multicolumn{2}{|r|}{$K_{2}^{*}(2050)$} \\
\hline Mode & $\overline{\Gamma_{i}(\mathrm{MeV})}$ & Amps. & $\Gamma_{i}(\mathrm{MeV})$ & Amps. \\
\hline 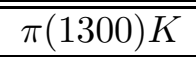 & 0 & ${ }^{1}{ }^{1} G_{4}=+0.0036$ & "0 & ${ }^{1} D_{2}=+0.0083$ \\
\hline$\eta(1293) K$ & 0 & ${ }^{1} G_{4}=+0.0038$ & 0 & ${ }^{1} D_{2}=+0.0083$ \\
\hline$\eta_{s}(1415) K$ & 0 & ${ }^{1} G_{4}=-0.0015$ & 0 & ${ }^{1} D_{2}=-0.0085$ \\
\hline$\rho(1465) K$ & 0 & ${ }^{3} G_{4}=+0.00046$ & 0 & ${ }^{3} D_{2}=-0.0035$ \\
\hline$\omega(1419) K$ & 0 & ${ }^{3} G_{4}=+0.0011$ & 0 & ${ }^{3} D_{2}=-0.0048$ \\
\hline$\pi K_{1}(1273)$ & 2 & $\begin{array}{l}{ }^{3} F_{4}=+0.017 \\
{ }^{3} H_{4}=+0.0036\end{array}$ & 79 & $\begin{array}{l}{ }^{3} P_{2}=+0.12 \\
{ }^{3} F_{2}=+0.017\end{array}$ \\
\hline$\eta K_{1}(1273)$ & 1 & $\begin{array}{l}{ }^{3} F_{4}=+0.021 \\
{ }^{3} H_{4}=+0.00073\end{array}$ & 22 & $\begin{array}{l}{ }^{3} P_{2}=+0.14 \\
{ }^{3} F_{2}=+0.0041\end{array}$ \\
\hline$\rho K_{1}(1273)$ & 0 & $\begin{array}{l}{ }^{3} F_{4}=-2.8 \cdot 10^{-6} \\
{ }^{5} F_{4}=+1.1 \cdot 10^{-5} \\
{ }^{3} H_{4}=+5.6 \cdot 10^{-9} \\
{ }^{5} H_{4}=+6.9 \cdot 10^{-9}\end{array}$ & 1 & $\begin{array}{l}{ }^{3} P_{2}=+0.023 \\
{ }^{5} P_{2}=-0.013 \\
{ }^{3} F_{2}=+0.00011 \\
{ }^{5} F_{2}=-0.00013\end{array}$ \\
\hline$\pi K_{1}(1402)$ & 2 & $\begin{array}{l}{ }^{3} F_{4}=-0.024 \\
{ }^{3} H_{4} \equiv 0\end{array}$ & 0 & $\begin{array}{l}{ }^{3} P_{2} \equiv 0 \\
{ }^{3} F_{2}=+0.0061\end{array}$ \\
\hline$\eta K_{1}(1402)$ & 0 & $\begin{array}{l}{ }^{3} F_{4}=-0.0035 \\
{ }^{3} H_{4}=-0.000079\end{array}$ & 7 & $\begin{array}{l}{ }^{3} P_{2}=-0.094 \\
{ }^{3} F_{2}=-0.0017\end{array}$ \\
\hline$\pi K_{2}^{*}(1429)$ & 2 & $\begin{array}{l}{ }^{5} F_{4}=-0.024 \\
{ }^{5} H_{4}=-0.0010\end{array}$ & 8 & $\begin{array}{l}{ }^{5} P_{2}=+0.043 \\
{ }^{5} F_{2}=+0.013\end{array}$ \\
\hline$\eta K_{2}^{*}(1429)$ & 0 & $\begin{array}{l}{ }^{5} F_{4}=+0.00081 \\
{ }^{5} H_{4}=+7.5 \cdot 10^{-6}\end{array}$ & 0 & $\begin{array}{l}{ }^{5} P_{2}=-0.0086 \\
{ }^{5} F_{2}=-0.00049\end{array}$ \\
\hline$\pi K^{*}(1414)$ & 0 & ${ }^{3} G_{4}=-0.0069$ & 0 & ${ }^{3} D_{2}=+0.0069$ \\
\hline$\eta K^{*}(1414)$ & 0 & ${ }^{3} G_{4}=-0.00086$ & 0 & ${ }^{3} D_{2}=+0.0062$ \\
\hline$\pi K(1460)$ & 0 & ${ }^{1} G_{4}=+0.0048$ & 0 & ${ }^{1} D_{2}=+0.0085$ \\
\hline$\eta K(1460)$ & 0 & ${ }^{1} G_{4}=-0.000027$ & 0 & ${ }^{1} D_{2}=-0.00069$ \\
\hline$\pi K^{*}(1717)$ & 0 & ${ }^{3} G_{4}=+0.00012$ & 0 & ${ }^{3} D_{2}=+0.010$ \\
\hline$\pi K_{2}(1773)$ & 0 & $\begin{array}{l}{ }^{5} D_{4}=+0.0038 \\
{ }^{5} G_{4}=+0.00013 \\
{ }^{5} I_{4}=+0.000017\end{array}$ & 61 & $\begin{array}{l}{ }^{5} S_{2}=+0.25 \\
{ }^{5} D_{2}=+0.013 \\
{ }^{5} G_{2}=+0.000083\end{array}$ \\
\hline$\pi K_{2}(1816)$ & 0 & $\begin{array}{l}{ }^{5} D_{4}=-0.0037 \\
{ }^{5} G_{4}=-0.000020 \\
{ }^{5} I_{4}=+2.9 \cdot 10^{-8}\end{array}$ & 0 & $\begin{array}{l}{ }^{5} S_{2}=+0.018 \\
{ }^{5} D_{2}=+0.0033 \\
{ }^{5} G_{2}=+0.000030\end{array}$ \\
\hline$\pi K_{3}^{*}(1776)$ & 0 & $\begin{array}{l}{ }^{7} D_{4}=-0.012 \\
{ }^{7} G_{4}=-0.00017 \\
{ }^{7} I_{4}=-9.3 \cdot 10^{-7}\end{array}$ & 0 & $\begin{array}{l}{ }^{7} D_{2}=+0.0066 \\
{ }^{7} G_{2}=+0.00012\end{array}$ \\
\hline & \multicolumn{2}{|c|}{$\Gamma_{\text {thy }}=98 \mathrm{MeV}$} & \multicolumn{2}{|c|}{$\overline{\Gamma_{t h y}}=295 \mathrm{MeV}$} \\
\hline & \multicolumn{2}{|c|}{$\Gamma_{\text {expt }}=198 \pm 30 \mathrm{MeV}$} & & \\
\hline
\end{tabular}




\begin{tabular}{|c|c|c|}
\hline \multicolumn{3}{|c|}{ Table K15. $1^{2 S+1} \mathrm{~F}_{3}$ kaons, general mixing (two states) } \\
\hline & $K_{3}^{a}(205$ & $=c\left|1^{1} \mathrm{~F}_{3}\right\rangle+s\left|1^{3} \mathrm{~F}_{3}\right\rangle$ \\
\hline Mode & $\Gamma_{i}(\mathrm{MeV})$ & Amps. \\
\hline$\rho K$ & $21.3 c^{2}+20.4 s c+24.2 s^{2}$ & $\begin{array}{l}{ }^{3} D_{3}=-0.0430 c-0.0496 s \\
{ }^{3} G_{3}=-0.0304 c+0.0263 s\end{array}$ \\
\hline$\omega K$ & $6.9 c^{2}+6.9 s c+7.9 s^{2}$ & $\begin{array}{l}{ }^{3} D_{3}=-0.0429 c-0.0496 s \\
{ }^{3} G_{3}=-0.0297 c+0.0258 s\end{array}$ \\
\hline$\phi K$ & $7.5 c^{2}-12.9 s c+9.4 s^{2}$ & $\begin{array}{l}{ }^{3} D_{3}=+0.0556 c-0.0642 s \\
{ }^{3} G_{3}=+0.0232 c+0.0201 s\end{array}$ \\
\hline$\pi K^{*}$ & $22.9 c^{2}-17.8 s c+25.4 s^{2}$ & $\begin{array}{l}{ }^{3} D_{3}=-.0430 c+.0497 s \\
{ }^{3} G_{3}=-.0336 c-.0291 s\end{array}$ \\
\hline$\eta K^{*}$ & $0.4 c^{2}+3.6 s c+17.4 s^{2}$ & $\begin{array}{l}{ }^{3} D_{3}=+0.0121 c+0.0816 s \\
{ }^{3} G_{3}=+0.00621 c-0.0313 s\end{array}$ \\
\hline$\eta^{\prime} K^{*}$ & $2.3 c^{2}+0.9 s c+0.1 s^{2}$ & $\begin{array}{l}{ }^{3} D_{3}=-0.0399 c-0.00791 s \\
{ }^{3} G_{3}=-0.00598 c+0.000889 s\end{array}$ \\
\hline$\rho K^{*}$ & $15.8 c^{2}+11.3 s^{2}$ & $\begin{array}{l}{ }^{3} D_{3}=-0.0500 c \\
{ }^{5} D_{3}=-0.0408 s \\
{ }^{3} G_{3}=-0.0156 c \\
{ }^{5} G_{3}=-0.0174 s\end{array}$ \\
\hline$\omega K^{*}$ & $5.0 c^{2}+3.6 s^{2}$ & $\begin{array}{l}{ }^{3} D_{3}=-0.0492 c \\
{ }^{5} D_{3}=-0.0402 s \\
{ }^{3} G_{3}=-0.0148 c \\
{ }^{5} G_{3}=-0.0166 s\end{array}$ \\
\hline$\phi K^{*}$ & $1.5 c^{2}+1.0 s^{2}$ & $\begin{array}{l}{ }^{3} D_{3}=-0.0351 c \\
{ }^{5} D_{3}=+0.0287 s \\
{ }^{3} G_{3}=-0.00355 c \\
{ }^{5} G_{3}=+0.00397 s\end{array}$ \\
\hline$b_{1} K$ & $0.9 s^{2}$ & ${ }^{3} F_{3}=-0.0140 s$ \\
\hline$h_{1} K$ & $0.5 s^{2}$ & ${ }^{3} F_{3}=-0.0178$ \\
\hline$h_{1}(1386) K$ & $0.1 s^{2}$ & ${ }^{3} F_{3}=-0.00756 s$ \\
\hline$a_{0}(1450) K$ & 0.0 & ${ }^{1} F_{3}=-0.00217 c+0.00260 s$ \\
\hline$a_{1} K$ & $1.3 c^{2}-2.8 s c+1.5 s^{2}$ & ${ }^{3} F_{3}=-0.0172 c+0.0180 s$ \\
\hline$f_{1} K$ & $0.2 c^{2}-0.5 s c+0.3 s^{2}$ & ${ }^{3} F_{3}=-0.0133 c+0.0142 s$ \\
\hline$f_{1}^{s \bar{s}}(1426) K$ & $0.1 s c$ & ${ }^{3} F_{3}=-0.00616 c-0.00686 s$ \\
\hline$f_{0}(1370) K$ & $-0.1 s c$ & ${ }^{1} F_{3}=-0.00494 c+0.00612 s$ \\
\hline$f_{0}^{s \bar{s}}(1500) K$ & 0.0 & ${ }^{1} F_{3}=-0.00116 c-0.00137 s$ \\
\hline$a_{2} K$ & $28.2 c^{2}+63.6 s c+36.7 s^{2}$ & $\begin{array}{l}{ }^{5} P_{3}=-0.0864 c-0.0997 s \\
{ }^{5} F_{3}=-0.0135 c+0.000215 \\
{ }^{5} H_{3}=-0.000662 c+0.000574 s\end{array}$ \\
\hline$f_{2} K$ & $11.1 c^{2}+24.7 s c+14.3 s^{2}$ & $\begin{array}{l}{ }^{5} P_{3}=-0.0883 c-0.102 s \\
{ }^{5} F_{3}=-0.0171 c+0.000327 s \\
{ }^{5} H_{3}=-0.00101 c+0.000873 s\end{array}$ \\
\hline$f_{2}^{\prime}(1525) K$ & $1.1 c^{2}-2.5 s c+1.5 s^{2}$ & $\begin{array}{l}{ }^{5} P_{3}=-0.0550 c+0.0635 s \\
{ }^{5} F_{3}=-0.000844 c-1.50 \cdot 10^{-6} s \\
{ }^{5} H_{3}=-4.63 \cdot 10^{-6} c-4.01 \cdot 10^{-6} s\end{array}$ \\
\hline
\end{tabular}




\begin{tabular}{|c|c|c|}
\hline \multicolumn{3}{|c|}{ Table K15. $1^{2 S+1} \mathrm{~F}_{3}$ kaons, general mixing (cont.) } \\
\hline & \multicolumn{2}{|r|}{$K_{3}^{a}(2050)$} \\
\hline Mode & $\Gamma_{i}(\mathrm{MeV})$ & Amps. \\
\hline$\rho(1465) K$ & $0.1 s c$ & $\begin{array}{l}{ }^{3} D_{3}=-0.00358 c-0.00414 s \\
{ }^{3} G_{3}=-0.000520 c+0.000451 s\end{array}$ \\
\hline$\omega(1419) K$ & 0.0 & $\begin{array}{l}{ }^{3} D_{3}=-0.00497 c-0.00573 s \\
{ }^{3} G_{3}=-0.00120 c+0.00104 s\end{array}$ \\
\hline$\pi K_{1}(1273)$ & $1.3 c^{2}+5.7 s c+6.1 s^{2}$ & ${ }^{3} F_{3}=-0.0161 c-0.0346 s$ \\
\hline$\eta K_{1}(1273)$ & $0.2 c^{2}+0.3 s c+0.1 s^{2}$ & ${ }^{3} F_{3}=-0.0109 c-0.0106 s$ \\
\hline$\rho K_{1}(1273)$ & $0.5 c^{2}+1.2 s c+0.7 s^{2}$ & $\begin{array}{l}{ }^{5} P_{3}=-0.0269 c-0.0311 s \\
{ }^{1} F_{3}=+0.0000525 s \\
{ }^{3} F_{3}=+0.0000321 c-0.0000556 s \\
{ }^{5} F_{3}=-0.000124 c-0.0000718 s \\
{ }^{5} H_{3}=-2.07 \cdot 10^{-7} c+1.80 \cdot 10^{-7} s\end{array}$ \\
\hline$\pi K_{1}(1402)$ & $0.9 c^{2}+0.9 s c+0.2 s^{2}$ & ${ }^{3} F_{3}=-0.0152 c-0.00706 s$ \\
\hline$\eta K_{1}(1402)$ & 0.0 & ${ }^{3} F_{3}=-0.00460 c+0.00354 s$ \\
\hline$\pi K_{0}^{*}(1412)$ & $0.5 c^{2}+1.3 s c+0.9 s^{2}$ & ${ }^{1} F_{3}=-0.0111 c-0.0147 s$ \\
\hline$\eta K_{0}^{*}(1412)$ & 0.0 & ${ }^{1} F_{3}=-0.00329 c+0.000675 s$ \\
\hline$\pi K_{2}^{*}(1429)$ & $31.8 c^{2}-69.6 s c+40.2 s^{2}$ & $\begin{array}{l}{ }^{5} P_{3}=-0.0890 c+0.103 s \\
{ }^{5} F_{3}=-0.0207 c-0.000460 s \\
{ }^{5} H_{3}=-0.00142 c-0.00123 s\end{array}$ \\
\hline$\eta K_{2}^{*}(1429)$ & $7.0 c^{2}+2.8 s c+0.3 s^{2}$ & $\begin{array}{l}{ }^{5} P_{3}=-0.103 c-0.0205 s \\
{ }^{5} F_{3}=-0.00452 c+3.81 \cdot 10^{-6} s \\
{ }^{5} H_{3}=-0.0000686 c+0.0000102 s\end{array}$ \\
\hline$\pi K^{*}(1414)$ & $0.4 c^{2}-0.1 s c+0.4 s^{2}$ & $\begin{array}{l}{ }^{3} D_{3}=-0.00713 c+0.00823 s \\
{ }^{3} G_{3}=-0.00715 c-0.00619 s\end{array}$ \\
\hline$\eta K^{*}(1414)$ & 0.0 & $\begin{array}{l}{ }^{3} D_{3}=+0.00110 c+0.00739 s \\
{ }^{3} G_{3}=+0.000170 c-0.000858 s\end{array}$ \\
\hline$\pi K^{*}(1717)$ & $0.1 s c+0.1 s^{2}$ & $\begin{array}{l}{ }^{3} D_{3}=-0.00350 c-0.00906 s \\
{ }^{3} G_{3}=-3.23 \cdot 10^{-6} c-0.000176 s\end{array}$ \\
\hline$\pi K_{2}(1773)$ & $0.1 s c+0.2 s^{2}$ & $\begin{array}{l}{ }^{5} D_{3}=-0.00489 c-0.0125 s \\
{ }^{5} G_{3}=-0.0000581 c-0.000193 s\end{array}$ \\
\hline$\pi K_{2}(1816)$ & 0.0 & $\begin{array}{l}{ }^{5} D_{3}=-0.00443 c+0.00195 s \\
{ }^{5} G_{3}=-0.0000332 c-0.0000111 s\end{array}$ \\
\hline$\pi K_{3}^{*}(1776)$ & $25.8 c^{2}-59.4 s c+34.2 s^{2}$ & $\begin{array}{l}{ }^{7} S_{3}=-0.165 c+0.190 s \\
{ }^{7} D_{3}=-0.0126 c+0.00722 s \\
{ }^{7} G_{3}=-0.000188 c-0.0000245 s \\
{ }^{7} I_{3}=-1.59 \cdot 10^{-6} c-1.37 \cdot 10^{-6} s\end{array}$ \\
\hline & $\Gamma_{\text {thy }}=20$ & $2-33 s c+239 s^{2} \mathrm{MeV}$ \\
\hline
\end{tabular}

